\title{
STUDY OF MAGNETOHYDRODYNAMIC EFFECTS FOR THE RICHTMYER-MESHKOV INSTABILITY
}

\author{
A Dissertation presented to \\ the Faculty of the Graduate School \\ at the University of Missouri
}

In Partial Fulfillment

of the Requirements for the Degree

Doctor of Philosophy

Wolfgang Justice Black

Dr. Jacob McFarland, Dissertation Supervisor

MAY 2019 
The undersigned, appointed by the Dean of the Graduate School, have examined the dissertation entitled:

\section{STUDY OF MAGNETOHYDRODYNAMIC EFFECTS FOR THE RICHTMYER-MESHKOV INSTABILITY}

presented by Wolfgang Justice Black, a candidate for the degree of Doctor of Philosophy and hereby certify that, in their opinion, it is worthy of acceptance.

Dr. Jacob McFarland

Dr. Hongbin Ma

Dr. Matthew Maschmann

Dr. Chung L. Chen

Dr. John Gahl 


\section{ACKNOWLEDGMENTS}

This work is the culmination of several years of academic and personal growth. None of this would be possible without my friend, mentor, and advisor Dr. Jacob McFarland. I am incredibly honored to be his first student and among the founding members of his laboratory. Dr. McFarland has provided me with immense opportunity and success beyond what the university could offer me. Without him I would not have received any graduate degrees, worked amongst some of the brightest scientists of this generation, nor would I have had such a successful start to my career. I must also thank the members of my committee, not just for their service on my committee but also their dedication and education throughout my academic career.

Dr. Maschmann has supported me, often providing a sounding board and pushing me to continue to work hard to accomplish my goals. Our morning coffee sessions are amongst my favorite memories at the University of Missouri.

Dr. Gahl was also there to lend me support and help me dissect the trickest of electrodynamic problems. No matter how simple the question, nor how often I'd ask the same thing a different number of ways, his patience and aid to my learning made this PhD work possible. I must acknowledge Dr. Chen as well, for his ability to challenge me and force me to strive harder and become a better fluid dynamicist. Without his crushing lessons and concise teachings, the fluids department at the University would not be nearly as rigorous. And lastly of my committee members, I must thank Dr. Ma. Dr. Ma allowed me to join his laboratory as an undergraduate research years ago, and without his initial support I would have never considered graduate school. Dr. Ma is among the few professors I've met who can see past a student, see their potential and give them the opportunity to excel. His academic 
prowess is only matched by his ability to lift every student up and encourage them to be more.

Outside of my committee there are too many people to thank. I have been supported by a massive network of friends and family. First, I must thank Jennifer Liu. Although our paths have diverged, the support she gave me through the entirety of my graduate career was often the only thing which kept me afloat. I'd be half the man I am now, and half the academic, if it wasn't for her. My friend Dr. Joe Tyler Rexwinkle also supported me, not just through graduate school but for most of my life. Dr. Rexwinkle was the one who encouraged me to go to college, and if I didn't have him as a constant friend I have no idea what my life would have become. Briefly, I want to thank all my friends who have supported me these last five-some years. Collin Powers, Caleb Roman, Kyle Cardine, Nathan Frey, Ted Hon, Sean Nobel, Alex England, Brenden Smith, Nick Thompson, Nick and Annie Wilkinson, and Kayla Wade to name a few.

I want to acknowledge my mother and step-father for their constant support. They always believed in me and my ability to complete whatever goals I put my mind too, even when I was too into the weeds to believe in myself. Similarly, my father has supported me and was also available to talk nerdy hobbies or philosophies and take my mind off the day-to-day stresses of graduate school.

For brevity, I want to thank my entire laboratory for all their support. Namely my cohort, Roy Allen, who fought with me against the beast that is plasma and magnetohydrodynamics. Without your expertise and help, I'm sure this dissertation would be a lot different. Similarly I'd like to thank John Middlebrooks, Curtis Maxom, Calvin Young, and Minoj Paudel. These friends and lab mates were my constant companions and shared with me the joys and sorrows of our basement laboratory.

In addition to my friends and family, I want to thank my mentors at the national 
laboratories. My often co-author and mentor Nicholas Denissen at Los Alamos National Laboratory has supported me for a number of years and was always willing to help me. His support, even in areas he was unfamiliar with, spawned alot of the work I've penned. Without him and his code knowledge, much of my work would not have been possible. I also want to thank Dr. Brandon Morgan and Dr. Lee Ellison of Lawrence Livermore National Laboratory. Dr. Morgan was my summer mentor for two summers, 2017 and 2018, and provided me the opportunity to grow at LLNL. His dogged pursuit of hydrodynamics forced me to grow and adapt and become a better scientist. Similary, my often theoretical discussions with Dr. Lee Ellision helped me to peice together the final chapter of this dissertation. Without his help this past year, this dissertation would feel incomplete. Of course, I'd also like to acknowledge both Los Alamos National Laboratory and Lawrence Livermore National Laboratory for the computational time, the academic resources, and the opportunities they provided me.

Lastly, I want to thank my new friends and co-workers at JUUL laboratory. They have made me feel at home in San Francisco, and have supported me both in my new career and in finishing my dissertation. I'd like to acknowledge Bill Alston, who without his support, finishing this dissertation would have been a dream. I also need to acknowledge Leana Tran-Le. Her support and encouragement gave me the motivation to finish in the past few months and without it, my life would surely be lesser.

Its thanks to all these people, and some I'm sure I've neglected to mention, that I was able to do the work presented in these pages and more. Without the support, encouragement, and faith of any one of these people I would have never been able to succeed or reach the end of my PhD program. So, with all my heart, thank you all. 


\section{TABLE OF CONTENTS}

ACKNOWLEDGMENTS .................. ii

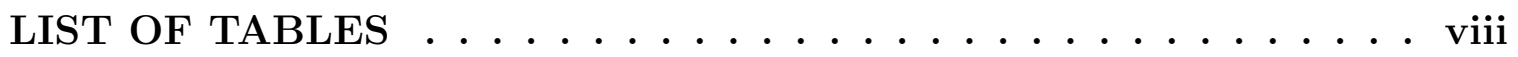

LIST OF FIGURES ................... ix

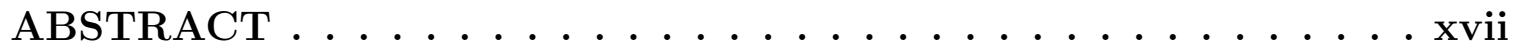

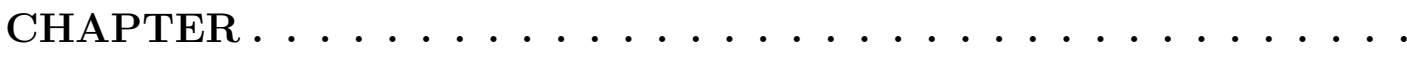

1 Introduction ...................... 1

1.1 Hydrodynamics . . . . . . . . . . . . . . . . . 2

1.1.1 Experiments and Simulations of the Richtmyer-Meshkov . . . 9

1.2 Magnetohydrodynamics . . . . . . . . . . . . . . 12

1.2.1 Literature Review for Plasma and Magnetohydrodynamics . . 20

2 The developement and characterization of an atmospheric nontransferred plasma source for magnetohydrodynamic experiments

2.1 Developement of an Atmospheric Plasma Torch for Investigating Magnetohydrodynamic Instabilities . . . . . . . . . . . . . . . . 29

2.1.1 Atmospheric-Pressure Plasma Torch Design . . . . . . . . . 31

2.2 Characterization of an Atmospheric Plasma Torch for Investigating Magnetohydrodynamic Instabilities . . . . . . . . . . . . . 35

2.2.1 The Langmuir Probe . . . . . . . . . . . . . . . 36

2.2.2 Spectral Methods . . . . . . . . . . . . . . . 38

2.2.3 Visual Methods for Hydrodynamic Experiments . . . . . . . . 42

2.3 Results........................... 47

2.3.1 Operating conditions of a nitrogen APPT . . . . . . . . . 47 
2.3.2 Langmuir Probe Results . . . . . . . . . . . . . . . . . . 52

2.3.3 2D Monochromatic Imaging Method Results . . . . . . . . . 55

2.3.4 Plasma Imaging Results . . . . . . . . . . . . . . . . . . 59

2.4 Conclusions . . . . . . . . . . . . . . . . . . . . . . 62

3 2D Simulations of the Magnetohydrodynamic Effects in a ShockAccelerated Gas Cylinder . . . . . . . . . . . . . 63

3.1 Computational Enviroment: FLAG . . . . . . . . . . . 65

3.1.1 Simulation Parameters and Initial Conditions . . . . . . 66

3.2 Results . . . . . . . . . . . . . . . . . . . . . . . . . . 69

3.2.1 Magnetic Field Orientation _ . . . . . . . . . . . . . . 69

3.2 .2 Magnetic Field Strength . . . . . . . . . . . . . . . . 81

3.2 .3 Incident Shock Wave Strength . . . . . . . . . . . . . . . 90

3.3 Conclusions . . . . . . . . . . . . . . . . . . . . 95

4 3D Simulations of the Cylindrical Magnetohydrodynamic RichtmyerMeskhov Instability . . . . . . . . . . . . . . . . . . 99

4.1 Computational Enviroment: Ares . . . . . . . . . . . . . . 100

4.1.1 Simulation Parameters and Initial Conditions _. . . . . . . 101

4.2 Results . . . . . . . . . . . . . . . . . . . . . . . 104

4.3 Conclusions . . . . . . . . . . . . . . . . . . . . . . . 116

4.3.1 Future Resistivity Work . . . . . . . . . . . . . . . 118

4.4 Acknowledgements . . . . . . . . . . . . . . . 120

5 Summary and concluding remarks $\ldots \ldots \ldots \ldots \ldots \ldots$

5.1 The developement and characterization of an atmospheric non-transferred plasma source for magnetohydrodynamic experiments . . . . . . . . . 123 
5.2 2D Simulations of the Magnetohydrodynamic Effects in a Shock-Accelerated Gas Cylinder . . . . . . . . . . . . . . . . . . . . . . 124

5.3 3D Simulations of the Cylindrical Magnetohydrodynamic RichtmyerMeskhov Instability . . . . . . . . . . . . . . . . . 127

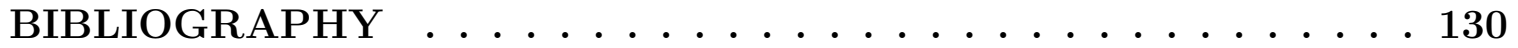

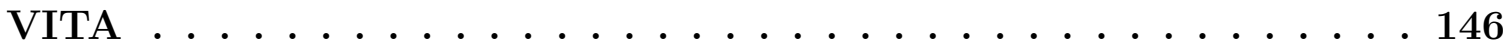




\section{LIST OF TABLES}

Table $\quad$ Page

2.1 Langmuir Circuit Operating Parameters . . . . . . . . . . . . 37

2.2 APPT Operating Parameters . . . . . . . . . . . . . . 47

3.1 Initial Conditions for 2D Simulations . . . . . . . . . . . 67

4.1 3D Simulation Domain and Mesh . . . . . . . . . . . . . 101

4.2 Initial Conditions for 3D Cylindrical Simulations . . . . . . . . . . . . 102 


\section{LIST OF FIGURES}

Figure

1.1 The pitchfork bifurcation illistrates how a flow can seem stable, then suddenly at some critical value $\left(R_{c}\right)$ bifurcation and go unsteady due to the existence of different flow solutions . . . . . . . . . . . .

1.2 A) A Kelvin-Helmholtz instability has formed between the clouds and the surrounding air, driving the clouds to approach turbulence. Photo credit: Grahamuk at the English Language Wikipedia B) This is a mid-time simulation of a Rayleigh-Taylor instability which has not yet transitioned to turbulence $[1] \ldots \ldots \ldots \ldots$

1.3 The evolution of a Richtmyer-Meshkov instability on a sinusoidal interface. A) The interface seperates a density gradient and is a perturbation which is misaligned with a pressure gradient. B) As the pressure gradient interacts with the interface baroclinic vorticity is deposited along the interface. C) The baroclinic vorticity drives the RM instability, transitioning the interface from linear growth and eventually into turbulence, $\mathrm{D}) \ldots \ldots \ldots \ldots \ldots \ldots \ldots \ldots \ldots$

1.4 paschen curves for various gases; as the pressure and/or gap distance is increased so to is the voltage required for breakdown for each gas

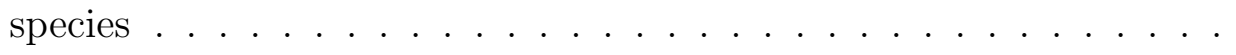


1.5 V-I characteristics for pre and post arc conditions. The arc occurs at

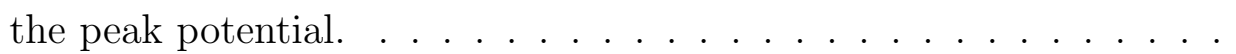

1.6 2D schematic of the cylindrical Richtmyer-Meshkov Instability driven by a shock wave in A) traditional hydrodynamics and B) with magnetohydrodynamic effects. . . . . . . . . . . . . . .

1.7 A 2D schematic representing the effects of the magnetic field orientation relative to the shock wave direction on vorticity. A) The magnetic field is parallel to the shock wave direction cause vortex translation parallel to the shock wave. B) The magnetic field is now perpendicular to the shock wave causing vortex translation transverse to the shock wave. In both magnetic field orientations, the site of maximum baroclinic vorticity deposition is $|\alpha|=p i / 2 \ldots \ldots \ldots$

1.8 Single Langmuir probe schematic: A metal wire probe is insulated in capillary glass, biased to some known DC voltage, $V_{B}$, and then inserted into the plasma. The potential difference between the plasma and the probe drives current into the probe, $i_{p}$, which is read by the DAQ. Resistors are used to help control the voltage at the measurement points as needed. This process is repeated to acquire data on the electric properties of the plasma . . . . . . . . . . . .

1.9 Idealized Langmuir probe data. Here one can see how the various properties can be extracted directly from the probe. . . . . . . . 
2.1 (A) APPT cut-out diagram 1) anode, 2) nozzle, 3) anode water cooling inlet, 4) anode water cooling outlet, 5) High current DC in (+), 6) axial gas (blue) inlet, 7) tangential gas (red) inlet, 8) alumina washers, 9) cathode water cooling inlet, 10) cathode water cooling outlet, 11) High current DC out (-), 12) thoriated tungsten cathode. (B) Top view of axial and tangential gas flow component. (C) Isometric cutout representation of axial (blue) and tangential (red) gas flow paths. . .

2.2 Langmuir circuit diagram. Here the tungsten probe with boroscilicate capillaruy glass is placed inside the plasma. The probe, set at some biased potential from the DC power supply, induces a current from the potential difference. This induced current is read by the LabJack U6 DAQ at the potential difference across $R_{5}$. The voltage dividers comprised of $R_{3}, R_{4}$ and $R_{1}, R_{2}$ are used to step down the voltage for acceptable levels for the DAQ . . . . . . . . . . . . .

2.3 Dissociated Nitrogen (N-I) and singly ionized Nitrogen (N-II) emission wavelengths vs intensity . . . . . . . . . . . . . .

2.4 A) Conceptual representation of solid particle PIV test with R-Z Laser Plane illuminated B) Constructed Mock-Tube for PIV testing . . . . 44

2.5 Comparison of the relative intensity at various wavelengths viewable through the (blue) Andover 532 $\pm 10 \mathrm{~nm}$ wavelength filter and the (red) Thor Labs $532 \pm 1 \mathrm{~nm}$ wavelength filter. The solid lines indicate manufacturers specifications while the shapes represent experimentally measured data. The N-I 528.12 nm emission line, desirable for the 2DMIM, is also shown here . . . . . . . . . . . . . . . . 
2.6 Calibration image (left) and APPT image (right) showing ionized region jet length (Region 1) at $20 \mathrm{~cm}$ and total jet length (Region 2) at

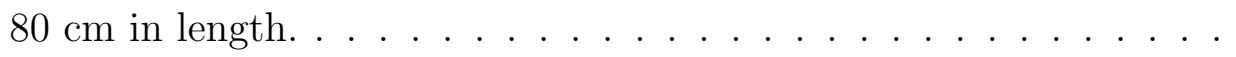

2.7 The voltage-current characteristics with respect to gas flow rate; as the current is increased the voltage increases as well . . . . . . . . . .

2.8 The total and ionized jet length with respect to increasing axial gas flow; as the gas flow increases the total and ionized region lengths converge. Similarly after a maximum axial flow rate of $\sim 6$ SLM both lengths begin to diminish due to turbulent effects . . . . . . . . . . . 50

2.9 Ionized and Total Jet lengths with respect to tangential gas flow rates

2.10 Langmuir probe data. These plots show the change in potential measured at the probe due to the current draw when $V_{B}=10 \mathrm{~V}$ (Red) and $V_{B}=-9 \mathrm{~V}$ (Blue). Here it can be seen that a positively biased probe draws negative current and decreases the overall potential while a negative probe draws positive current and increases the overall potential while the probe enters the plasma . . . . . . . . . .

2.11 Top: Raw Langmuir Data, Bottom: The log natural of translated Langmuir data. The data has been shifted up by the estimated ion saturation current in an effort to find the electron temperature. Here the data can be seen to be scattered and difficult to correlate with regards to the idealized langmuir data previously shown . . . . . . .

2.12 The 2DMIM results showing the monochromatic raw image (Left), the $R-Z$ temperature distribution (Middle), and the degree of ionization as a function of temperature (Right) for axial and tangential flow rates of 2.1 and 0.9 SLM respectively and an arc current of $200 \mathrm{~A}$. . . . 
2.13 Radial Temperature profile at nozzle exit for APPT experiment shown in Fig. 2.12. Dashed line indicates plasma temperature measurements with unresolvable N-I concentrations . . . . . . . . . . . . .

2.14 Plasma-Particle Interface Imaging results: a) $R-Z$ plane imaged without any filter b) $R-Z$ plane imaged with $532 \pm 10 \mathrm{~nm}$ Andover bandpass filter c) $R-Z$ plane imaged with $532 \pm 1 \mathrm{~nm}$ Thor Labs bandpass filter d) $R-\theta$ plane imaged with 532 Thor Labs bandpass filter. Both c) and d) give the best results for eliminating the emissions from the plasma for future hydrodynamic experiments . . . . . . . . . . . .

3.1 2D species pseudo-color representing all initial conditions for this work. Here the shock wave is traveling in the $+Y$-direction and the interface considered as a $2 \mathrm{~cm}$ diameter cylinder of $N_{2}$ at $T=2500 \mathrm{~K}$. The magnetic field, if present may be parallel (along Y) or perpendicular (along $\mathrm{X}$ ) to the direction of propagation of the shock and is not shown here. The image is centered about $y=0 . \ldots . . . . . . . .668$

3.2 Magnetic orientation study; pseudo-color of species for the $M 1.7 B 0$, $M 1.7 B_{\perp} 50$, and $M 1.7 B_{\|} 50$ cases with respect to $\tau$. The images are centered about $y$, respective to $\tau$ at $y(\tau \approx 3)=3.11 \mathrm{~cm}, y(\tau \approx 7)=$ $7.64 \mathrm{~cm}$, and $y(\tau \approx 15)=15.55 \mathrm{~cm}$. . . . . . . . . . . . 71

3.3 Mixedness, $\Xi$, with respect to $\tau$ for the magnetic orientation study. . 73

3.4 Late time pseudo-colors for $\omega$ and $\Xi$ for the magnetic orientation study. The images are centered about $y$, respective to $\tau$ at $y(\tau \approx 3)=3.11$ $\mathrm{cm}, y(\tau \approx 7)=7.64 \mathrm{~cm}$, and $y(\tau \approx 15)=15.55 \mathrm{~cm} . \ldots . . . . .76$

3.5 The total and reduced area (local) enstrophy for the $M 1.7 B 0, M 1.7 B_{\perp} 5$, and $M 1.7 B_{\|} 5$ cases. . . . . . . . . . . . . . . 
3.6 The correlation coefficient with respect to $\tau$ for the magnetic orientation study. . . . . . . . . . . . . . . . .

3.7 Magnetic strength study; Pseudo-color of species for the parallel magnetic field cases with respect to $\tau$. The images are centered about $y$, respective to $\tau$ at $y(\tau \approx 3)=3.11 \mathrm{~cm}, y(\tau \approx 7)=7.64 \mathrm{~cm}$, and $y(\tau \approx 15)=15.55 \mathrm{~cm} . \ldots \ldots \ldots$

3.8 Magnetic strength study; pseudo-color of species for the perpendicular magnetic field cases with respect to $\tau$. The images are centered about $y$, respective to $\tau$ at $y(\tau \approx 3)=3.11 \mathrm{~cm}, y(\tau \approx 7)=7.64 \mathrm{~cm}$, and $y(\tau \approx 15)=15.55 \mathrm{~cm} . \ldots \ldots \ldots \ldots$

3.9 The mixedness for the magnetic strength study. Here $B 0$ is included to highlight the effects of the magnetic field. . . . . . . . . . . 86

3.10 Late time vorticity field for the magnetic strength study. The images are centered about $y(\tau \approx 15)=15.55 \mathrm{~cm} . \ldots \ldots$. . . . . . .

3.11 The correlation coefficient, given as Eq. 3.6, for the magnetic strength

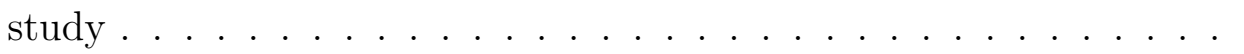

3.12 The late time species and vorticity plots for the incident shock wave strength study. The species pseudo-colors are all on the same scale shown on the right of the image while the vortices are Mach number specific and shown below each group. The images are centered about $y$, respective to $M$ at $y(M=1.2)=7.60 \mathrm{~cm}, y(M=1.7)=15.55 \mathrm{~cm}$, and $y(M=2.2)=19.40 \mathrm{~cm} \ldots \ldots \ldots \ldots$

3.13 The mixedness for the incident shock wave study. Small dash lines indicate $M=1.22$, solid lines indicate $M=1.66$, and large dash lines indicate $M=2.2$; while black represents cases without a magnetic field and blue represents cases with a perpendicular magnetic field of 500 G. 94 
3.14 The correlation coefficients for the incident shock wave study. Small dash lines indicate $M=1.22$, solid lines indicate $M=1.66$, and large dash lines indicate $M=2.2$; while black represents cases without a magnetic field and blue represents cases with a perpendicular magnetic

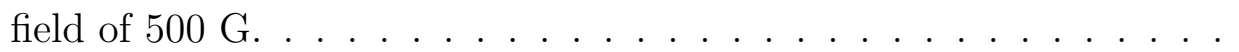

4.1 Initial conditions for all 3D simulations presented in Chapter 4. A) Contour plot with the cylindrical interface (red) and the shock wave (black) annotated for size and magnitude, here it can be seen that the shock wave travels in $\mathrm{X}$ B) $\mathrm{X}-\mathrm{Y} 2 \mathrm{D}$ pseudo-color slice for species $\mathrm{C}$ ) $\mathrm{X}-\mathrm{Z} 2 \mathrm{D}$ pseudo-color slice for species, in both of B) and C) the shock wave is represented by a black line . . . . . . . . . . . . . . 103

4.2 Contour plots for the interfacial evolution of $B 0, B_{\|}$, and $B_{\perp}$ cases, where a red surface is meant to represent the $5 \%$ species contour. The mid-plane species pseudo-color is included for $B 0$ as reference. Both the magnetic field cases show representative contours for Alfven waves, shown as semi-transparent black surfaces . . . . . . . . . . . . . 106

4.3 Mid-plane $(\mathrm{Z}=2.5 \mathrm{~cm})$ Pseudo-color for temperature with respect to post shock time . . . . . . . . . . . . . . . . . . . 108

$4.42 \mathrm{D}$ vorticity pseudo-colors at various $\mathrm{Z}$ planes for $B 0$. . . . . . . . . 109

$4.52 \mathrm{D}$ vorticity pseudo-colors at various $\mathrm{Z}$ planes for $B_{\|} \quad \ldots \ldots$. . . . 110

$4.62 \mathrm{D}$ vorticity pseudo-colors at various $\mathrm{Z}$ planes for $B_{\perp} \ldots \ldots$. . . . . 112

4.7 The total enstophy calculated across the entire domain for B0 (Black), $B_{\|}($Red $)$, and $B_{\perp}($ Blue $) \ldots \ldots \ldots \ldots$ 
4.8 The total enstophy (solid), enstrophy in X (dash-dot), enstrophy in Y (small dash), and the enstrophy in Z (large dash) calculated across the entire domain for $B 0$ (Black) and $B_{\perp}$ (Blue) . . . . . . . . . . . . . 114

4.9 The correlation coefficient, indicative of when mixing occurs and the scales of mixing, for $B 0$ (Black), $B_{\|}$, and $B_{\perp} \ldots \ldots \ldots 117$

4.10 The magnetic diffusion time with respect to degree of ionization for various collisional frequencies . . . . . . . . . . . . . . . . . 121 


\begin{abstract}
This work presents experimental and computational studies of the RichtmyerMeshkov (RM) instability with Magnetohydrodynamic (MHD) effects. The experimental work does not consider the instability or its growth, but rather developes an atmospheric plasma jet for use in future magnetohydrodynamic experiments. The operating conditions of the torch are explored to optimize the ionized length of the plasma jet by varying the voltage-current characteristics and the gas flow rates. Probe, spectral, and visual diagnostics are also developed in an effort to characterize the plasma. The probe diagnostics were unsuccessful but discussions are included to help improve the technique. The visual Mie-Scattering like technique is able to capture qualitative images of the plasma flow field and are ready for use in future hydrodynamic experiments where the qualitative growth is of interest.

Simulations utilized the hydrocode FLAG, developed at Los Alamos National Laboratory, are performed on a 2D shock cylinder plasma-air interface where MHD effects work to remove vorticity from the interface and suppress RM growth. To study this magnetic field orientation, magnetic field strength, and incident Mach number are all varied in this study. It was found that the orientation of the magnetic field relative to the shock wave direction causes different morphology and can effect the amount of observable RM suppression. Similarly, increasing the magnetic field strength reduces the effects of the baroclinic vorticity, responsible for RM growth, by generating strong MHD waves which carry the vorticity away from the interface quicker. Increasing the Mach number can also cause varying qualitative effects, with greater Mach numbers showing greater interfacial compression. But comparing the MHD RM to the RM instability at a single Mach number still shows suppresion of the instability.
\end{abstract}


Finally a 3D cylindrical interface is simulated using the hydrocode ARES. These simulations compare the cylindrical Richtmyer-Meshkov to two cases of the MHD-RM instability; one with a parallel and one with a perpendicular magnetic field of 500 Guass. As per literature, the magnetic cases exhibit suppression through decreased enstrophy, vorticity, and mixedness with respect to time in addition to the clear morphological differences. 


\section{Chapter 1}

\section{Introduction}

This document details research performed towards the completion of a Doctoral degree in Mechanical Engineering. The work presented here is divided into four chapters which cover background, experimentation, and simulation efforts towards understanding magnetohydrodynamic effects on shock driven instabilities, with experimental and 3D simulation studies of the Richtmyer-Meshkov (RM) Instability. Interest in the RM instability stems from a decades old desire to understand and support fusion experiments. The experiments and simulations assume a low energy university laboratory setting. However, the physics considered have strong applications towards the high energy density systems often found in astrophysics and national laboratory experiments. These physics include the generation of plasma, diagnostics for plasma characterization, shock driven hydrodynamics, multispecies/multiphase mixing, the presence of a magnetic field, and magnetic tension. A literature review for these topics encompassing previous theoretical, experimental, and simulation work is included in this introduction chapter. In Chapter 2 the developement and diagnosis of an atmospheric plasma torch is presented, and is closely based off of a publica- 
tion the author was closely involved in [2]. This sets up an interface to be used in future magnetohydrodynamic experiments to be done by the next generation of masters and doctoral students at the University of Missouri Fluid Mixing and Shock Tube Laboratory (MU-FMSTL). Chapters 3 and 4 detail simulation work performed using highly parallelized multiphysics hydrodynamics codes. First, Chapter 3 shows 2D simulations of an ideal magnetohydrodynamic shock-bubble problem has been published in Physical Review Fluids [3]. Chapter 4 presents 3D simulations of the Magnetohydrodynamic Richtmyer-Meshkov (MHD-RM) Instability. While this work consideres similar physics as Chapter 3 different numerical methods were explored and the calculations were taken into 3D in preparation to incorporate resistive effects for future work.

\subsection{Hydrodynamics}

In the study of fluid dynamics there exists several flow regimes. The most commonly studied are steady laminar flow which is relatively easy to analyse, and turbulent flow, often associated with statistical analysis and chaos mathematics. However, there is a rich space between the two regimes where the flow may transition from laminar into turbulent flow. This transition can occur due to a hydrodynamic instability (HI). Flow may be considered stable if it experiences a small perturbation but then returns to its pre-disturbed state or assumes a new steady configuration. However, if the perturbation grows in amplitude until it departs from its initial state and does not recover, then the flow is said to have experienced a hydrodynamic instability [4].

A hydrodynamic instability occurs when a flow experiences a series of bifurcations which carry it from its original stable state to one which shares no recognizable features. The points of bifurcations often occur at critical Reynolds numbers which 


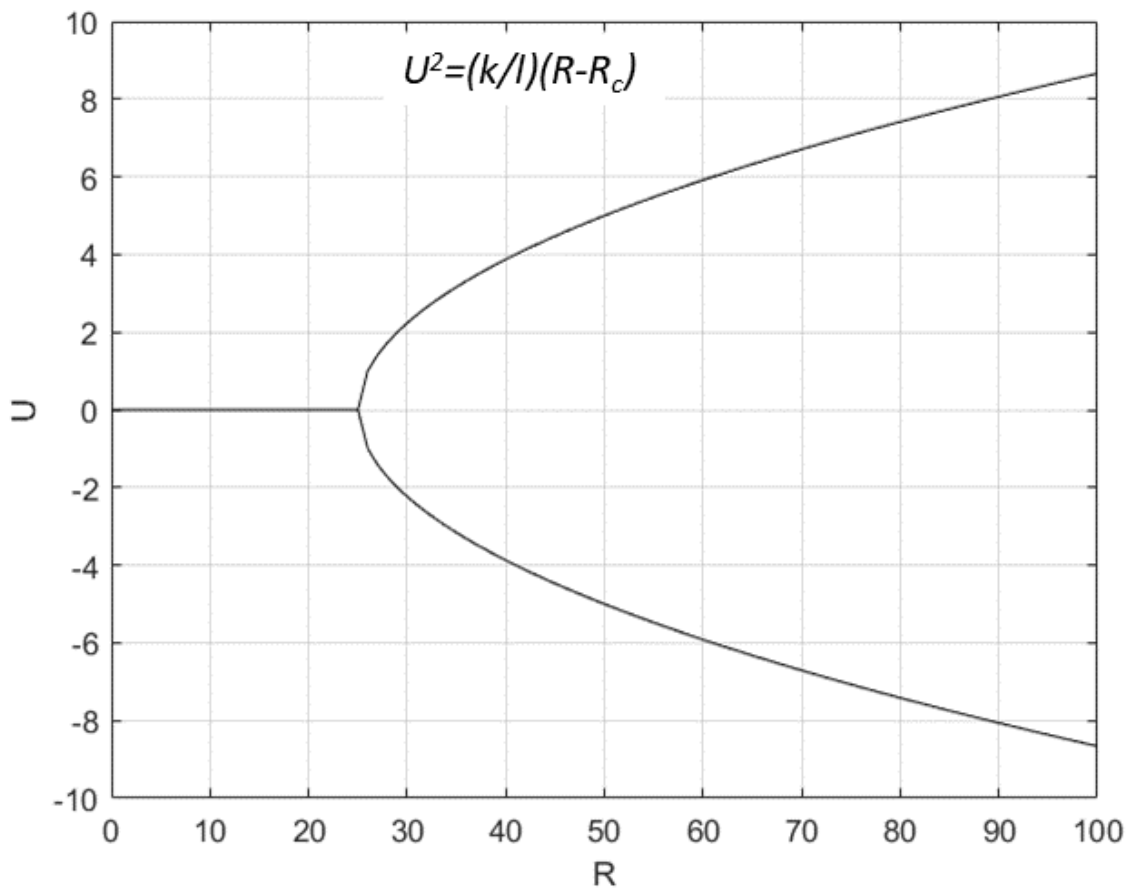

Figure 1.1: The pitchfork bifurcation illistrates how a flow can seem stable, then suddenly at some critical value $\left(R_{c}\right)$ bifurcation and go unsteady due to the existence of different flow solutions

depend on the kind of flowing being analyzed. For example, consider the well-known pitchfork bifurcation [4]. This can be modeled using Eq. 1.1. Here, $a=k\left(R-R_{c}\right)$ and is a function of $k, R$, and $R_{c}$ or the wavenumber, the Reynolds number, and the critical Reynolds number; $U$ is the flow velocity. This equation has three solutions: $U=0$ and $U= \pm a / l$ if $a / l>0$. Here one can see there is a "pitchfork" bifurcation $R=R_{c}, U=0$, illustrating that at $R=R_{c}$ the solution breaks symmetry. That is for $k l>0$ then there is a single unique solution $(U=0)$ for all $R<R_{c}$. However, there are two solutions for $R>R_{c}$ which continue to grow in amplitude, as shown in Fig. 1.1.

$$
a U-l U^{3}=0
$$


Unstable flow experiences bifurcations driving it to a state where it is difficult to predict its behavior with respect to time. While the previous example was simplified to a single bifurcation of three potential solutions, hydrodynamic instabilities can undergo copious bifurcations until they reach a truly turbulent state. Some of the most commonly studied hydrodynamic instabilities include the Kelvin-Helmholtz (KH) instability [5, 6], the Rayleigh-Taylor (RT) instability [7, 8], and the RM instability $[9,10]$. These are often studied together, as the RM may drive secondary RT and KH instabilities. While this work focuses on magnetohydrodynamic effects on the RM instability, some background on the KH and RT instabilities can be informative. The KH instability occurs when a shear layer develops along some interface between fluid layers. If surface tension effects are ignored, as is often done when studying gases, then the instability will develop regardless of the velocity difference. This is true in a two-fluid system or in a homogenous fluid, so long as there exists some perturbation separating regions of different velocities. However, if surface tension is included then small perturbations will be neglected and the two-fluid interface will be considered as marginally stable. The $\mathrm{KH}$ can also be stabilized across density gradients in a homogenous fluid or in a two-fluid system[4, 11].

Figure 1.2 A) shows an example of an atmospheric KH instability. Here a cloud-air interface has provided some pertubration between a density/shear layer and the resulting interface has experienced the KH instability forming wave-like structure which appear almost single-modal. As the KH continues to grow along the perturbation it begins to break down the single mode into a more multi-modal structure and thus the system approachs turbulence. The arrow over the image indicates the direction in which this transition is occuring.

The RT instability, Fig. 1.2 B) [1], is another instability which has a dependency upon density gradients. The RT instability manifests within systems where an ac- 


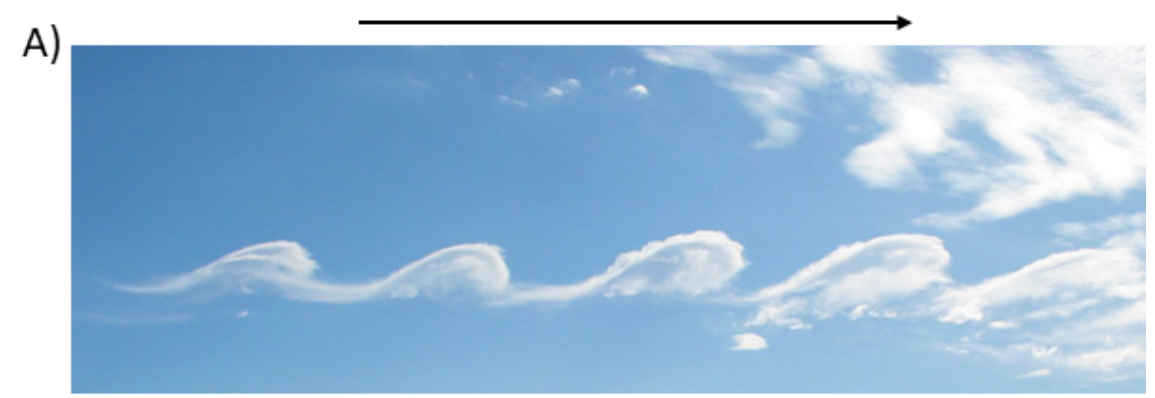

B)

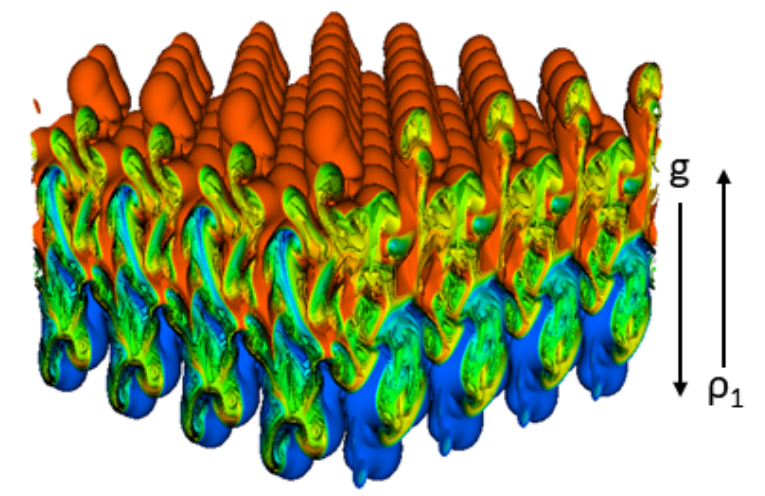

Figure 1.2: A) A Kelvin-Helmholtz instability has formed between the clouds and the surrounding air, driving the clouds to approach turbulence. Photo credit: Grahamuk at the English Language Wikipedia B) This is a mid-time simulation of a RayleighTaylor instability which has not yet transitioned to turbulence [1] 
celeration drives a lighter fluid into a heavier fluid. If one thinks of the ocean when trying to understand hydrodynamics they may initially picture the waters surface interacting with the air. If there exist some current in the water, it's not impossible to image a KH instability developing at this interface; similar to the instability in Fig. 1.2 A). However, this schematic can not support RT instability. If we could somehow freeze time for the water and air interface and place the water over the air such that gravity pulled the water down through the air (or boyancy drove the air up through the water) then a RT interface would develop along some small perturbation at the interface.

In nature the RT instability is often seen in mushroom clouds, atmospheric sciences, and in astrophysics. Figure 1.2 B) shows simulation results of a single mode RT instability forming mushroom-like structures, commonly associated with the RT and RM instabilities [9]. The arrows here indicate that gravity is acting downward, against the density gradient and thus the system is RT unstable.

When studying $\mathrm{HI}$ it is of interest to predict how the system may evolve with respect to time or initial perturbation. This can quickly go nonlinear as the system approaches turbulence, but the linear regime can be studied via linear stability theory and is helpful in understanding early HI growth. The linear growth rate of the RT instability can be calculated by Eq. 1.2 [12]. Where $S$ is the amplitude of the perturbation between the interface, $t$ is the physical time for the system, and $n$ is the growth factor. The growth factor, shown in Eq. 1.3, is dependent upon $g, A, k, \psi, \nu$, and $D$ or the acceleration driving the system, the Atwood number, the wavelength of the perturbation, a diffusion thinkness, the viscosity, and the diffusivity of the fluids. Equation 1.4 give the Atwood number as a function of the densities across the interface with the subscripts 1 and 2 representing the light and heavy fluids respectively. Equation 1.5 gives the diffusivity as a function of the Schmidt number. 
These parameters are both heavily dependent upon the fluids, or the thermodynamic state of the homegenous fluid, being considered. Equation 1.6 is based off experiments and is thought to be heavily dependent upon the perturbation and the fluids involved, however the form shown here is typically used in literature for a sinusoidal interface $[13,12]$.

$$
\begin{gathered}
S=\exp (n t) \\
n=\left(\frac{g A k}{\psi}+\nu^{2} k^{4}\right)^{1 / 2}-(\nu+D) k^{2} \\
A=\left|\frac{\rho_{2}-\rho_{1}}{\rho_{2}+\rho_{1}}\right| \\
D=\frac{\nu}{S c} \\
\psi=1+0.374 D k
\end{gathered}
$$

Traditionally the growth factor, Eq. 1.3, may be considered without viscous contributions. However, by considering these effects one may find a wavelength which will grow rapidly and drive the system to turbulence faster than other wavelengths. Likewise there will also exist some wavelength at which the perturbation amplitude will not grow; nor will any greater wavelength beyond this value grow to turbulence. This is similar to how surface tension or density gradients may work to dampen or stablize a potential KH instability.

The Richtmyer-Meshkov instability is similar, and sometimes considered a special 
case of, the RT instability. Instead of a constant acceleration the RM instability evolves from an impulsive acceleration. This changes $g$ in Eq. 1.3 to an impulse function, which changes from an initial value of 0 , some non-zero value, and back to 0. In this way the stability of a light fluid over a heavy fluid is removed; allowing the RM to evolve in systems that are RT stable. Since the RM can grow regardless of which fluid is 'on top', or is accelerated into the other fluid, the subscripts 1 and 2 in Eq. 1.4 now refer to the first fluid and the second fluid to be accelerated.

While equations 1.2 and 1.3 can help to describe how the amplitude of the interface evolves linearly it does not reveal the mechanism which acts to drive the systems RT or RM unstable. These HI result from three main ingredients: a pressure gradient, a density gradient, and some misalignment between them. The pressure gradient, provided by an impulsive acceleration for the RM, propagates through the misaligned density gradient and deposits vorticity. The dependence upon the interaction of these ingredients can be seen in the baroclinic term in the vorticity conservation equation, Eq. 1.7. The vorticity, $\omega$, is a function of $u, \nu, \rho$, and $P$, or the velocity, kinematic viscosity, density, and pressure while $t$ denotes time. This vorticity then acts to stretch the area of the interface, or the density gradient, and rapidly increases the mixing; driving a stable interface to eventually transition toward decaying turbulent mixing.

$$
\frac{\partial \vec{\omega}}{\partial t}+(\vec{u} \cdot \nabla) \vec{\omega}=(\vec{\omega} \cdot \nabla) \vec{u}-\vec{\omega}(\nabla \cdot \vec{u})+\left[\frac{1}{\rho^{2}} \nabla \rho \times \nabla P\right]_{\text {baroclinic }}
$$

The amount of vorticity deposition is directly related to the strength of the pressure and density gradients. The strength of the pressure gradient, often provided by a shock wave, can be represented by the Mach number, $M=u_{1} / a$, with a larger 
$M$ depositing more vorticity. $M$ is a function of the fluid velocity over the speed of sound in that fluid, $a$. The density gradient is given by the Atwood number, Eq. 1.4, shown above. Just as $M$ and $A$ represent the strength of the pressure and density gradients, the misalignment of the two can be considered as the linearity of the interface, $\eta_{0} / \lambda$, or the amplitude of the interface over the wavelength. It was shown by Richtmyer's [9] linear model, Eq. 1.8, that the lower this ratio is the weaker the instability that will form and vice-versa. In Eq. $1.8 n, k, g$, and $A$ represent the amplitude of the interface, the wavelength, the impulsive acceleration, and the Atwood number respectively.

$$
\frac{\partial^{2} n}{\partial t^{2}}=k g n A
$$

The dependency of the these three ingredients is shown in Fig. 1.3. Here a sinusoidal interface seperates two fluids providing a density gradient. A planar pressure gradient, here a shockwave represented by a line, moves towards the interface. After the two interact a transmitted wave moves across the density gradient into the second fluid depositing vorticity along the interface. This baroclinic vorticity works to stretch and deform the interface transitioning the interface through the linear RM regime, into nonlinear growth, and eventually into turbulence.

\subsubsection{Experiments and Simulations of the Richtmyer-Meshkov}

To better understand the RMI, experimentalists have studied various interface perturbations, species for the density gradient, and methods to create pressure gradients. While the most common way to create the pressure gradient experimentally is in a mechanical shock tube, which utilizes the release of high pressure into a lower pressure region; both laser-driven shock waves [14] and gravity-driven sleds $[15,16]$ have been 


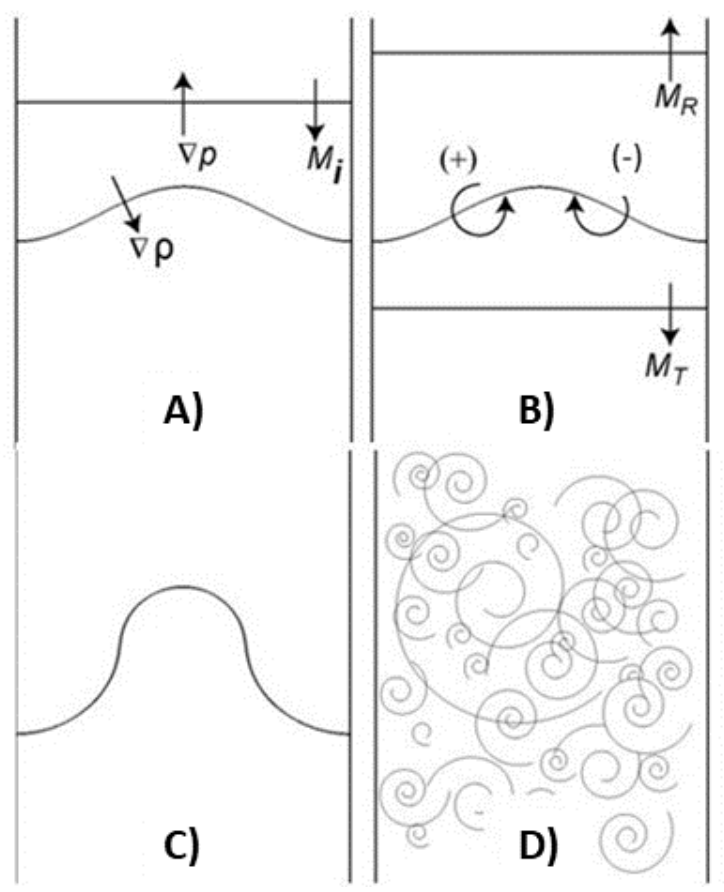

Figure 1.3: The evolution of a Richtmyer-Meshkov instability on a sinusoidal interface. A) The interface seperates a density gradient and is a perturbation which is misaligned with a pressure gradient. B) As the pressure gradient interacts with the interface baroclinic vorticity is deposited along the interface. C) The baroclinic vorticity drives the RM instability, transitioning the interface from linear growth and eventually into turbulence, D) 
utilized. Experimentalists have also studied a wide range of interface configurations. Gas-bubble interactions have been used to deposit a bubble of $\rho_{2}$ into a shock tube containing a gas at $\rho_{1}[17]$. Similarly, other groups have flown gas cylinders into a shock tube to create the density gradient $[18,19,20]$. Spherical and cylindrical interfaces both create a two-interface system as the shock wave must travel from one density, into the sphere/cylinder, and back out into the original fluid, and thus share similar theory. The work presented within chapters 3 and 4 will consider a cylindrical interface in a shock tube environment through simulations. More traditional wave-like interfaces have been studied as well, where some groups have generated a sinusoidal interface $[21,22,23]$ or used the shear-flow KH instability [24]. A half wavelength like inclined interface was also studied extensively experimentally and in simulations $[25,26,27,28]$.

In addition to experiments many groups have explored the RM through simulations. While much of this research focuses on the RM instability without MHD effects, these methods and codes are often used to inform researchers on fusion applications and astrophysics [29, 30]. Many of these codes solve the hydrodynamics through nominally second-order large-eddy simulations utilizing Eulerian [31, 32, 33] and arbitrary Lagrangian-Eulerian schemes [27, 34] for a wide range of perturbations and initial conditions. Other have used higher order methods [35, 36, 37].

In much of the research the RM instability is considered to occur between some fluid layers. It's common to assume the fluids as ideal gases to simplify the analysis. As such the fluids and plasma discussed in this work are considered as ideal gases using the equation of state shown in Eq. 1.9, where e is energy. Equations 1.10 through 1.19 are also used for the continuity, momentum, and energy of the fluids.

$$
P=\rho(\gamma-1) e
$$




$$
\begin{gathered}
\frac{\partial \rho}{\partial t}-\nabla(\rho \vec{u})=0 \\
\rho\left(\frac{\partial}{\partial t}+\vec{u} \cdot \nabla\right) \vec{u}=-\nabla P+\nabla \cdot \vec{\tau}+\rho \vec{g} \\
\frac{\partial e}{\partial t}+\nabla[(e+P) \vec{u}]=0
\end{gathered}
$$

\subsection{Magnetohydrodynamics}

As high energy density (HED) research continues to grow, magnetohydrodynamics (MHD) has become an important research field due to its presence in various applications. In astrophysics, where the physical medium is typically ionized and can experience magnetic fields, the inclusion of MHD effects can provide physicists with greater insight into cosmological evolution [38, 39]. Engineered HED systems, like Inertial Confinement Fusion (ICF) studied at the National Ignition Facility (NIF), typically experience an array of complex physical phenomena from shock driven accelerations to solid-plasma phase change. The transition to a plasma state alone encourages the consideration of MHD, but some systems may be further complicated by externally applied magnetic fields. For example, experiments performed at the Laboratory for Laser Energetics utilizing the Omega Laser on NIF capsules incorporated an external magnetic field [40], thus exhibiting MHD interactions between the plasma and the magnetic field. A promising potential alternative to ICF is Magnetized Liner Inertial Fusion (MagLIF ), where a target undergoes magnetically driven compression and then is laser heated to extreme temperatures while being magnetically confined $[41,42]$. Similar to the NIF and OMEGA experiments, MagLIF also 
experiences shock driven hydrodynamics, phase change to plasma, and an external magnetic field; thus, understanding how magnetohydrodynamics affect these instabilities can provide much needed insight into improving the experimental yield of these processes $[43,44,45]$.

In much of the research directed towards HED systems, however, a classic hydrodynamics approach is often taken. While this can provide important insight, once the system transitions into a plasma state the necessary physics required to accurately understand the system also transition from hydrodynamics into the study of electrically conducting fluids. Its important then to consider how nature may transition from hydrodynamics into MHD. One method to produce a plasma, formed by ionizing a gas, is to flow an electrical current through a normally insulating fluid. This current, induced by an electric potential between two electrodes, forms a discharge. To induce a discharge, a self-sustaining arc must first be generated between the electrodes. The electric potential required for the arc depends on the distance between the electrodes, $d$, the gas species, and the system pressure, $P$. This complex process is known as electric breakdown [46]. The breakdown of a gas is governed by Paschen's Law, Eq. 1.13. The voltage at which breakdown occurs, or $V_{B D}$, is a function of $A, \gamma_{s e}$, and $B$ or the saturation ionization in the gas at a particular electric field to pressure ratio, the secondary-electron-emmision coefficient, and the excitation and ionization energies. These values are often tabulated. Using Eq. 1.13 the breakdown voltage as a function of pressure and electrode gap can be plotted for multiple gas species. These are shown in Fig. 1.4 and are known as Paschen curves.

$$
V_{B D}=\frac{B(P d)}{\ln (A(P d))-\ln \left(1+1 /\left(\gamma_{s e}\right)\right)}
$$

From this figure it can be seen that if the gap distance is held cosntant then as 


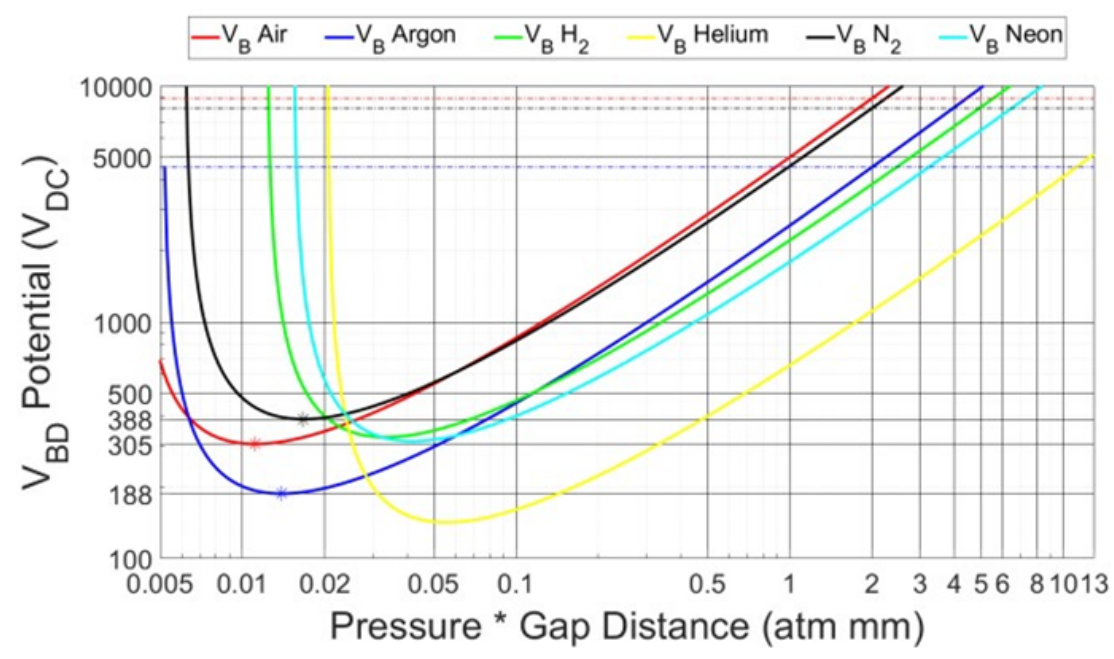

Figure 1.4: paschen curves for various gases; as the pressure and/or gap distance is increased so to is the voltage required for breakdown for each gas species

pressure increases so does the required breakdown voltage. Similarly if pressure is constant, then increasing gap distance increases the necessary breakdown voltage. Once the required breakdown voltage is achieved a spark forms. To generate a selfsustaining plasma the spark must transition into an arc. This transition is achieved nearly instantaneously at atmospheric pressure when a large current is induced between the electrodes, resulting in a self-sustaining arc discharge that ionizes the fluid and forms a plasma. The voltage-current (VI) characteristics pertaining to the regions of electric breakdown, spark formation, and arc establishment are shown in Fig. 1.5. For atmospheric-pressure plasmas, the electric potential between the anode and cathode increases to the threshold value of the breakdown voltage, from the left to the peak of the curve in Fig. 1.5. Once transition to an arc occurs, the required voltage decreases rapidly as the current increases rapidly [46].

Considering the additional physics in electrically conducting fluids, or plasma, is even more important in the presence of an external magnetic field, where MHD 


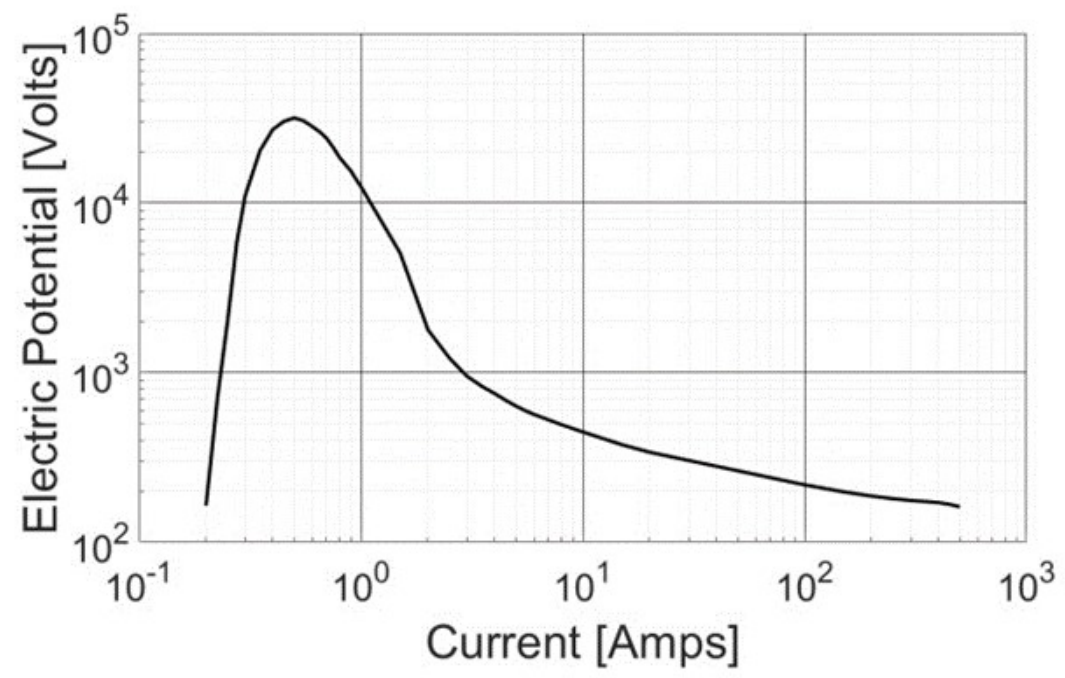

Figure 1.5: V-I characteristics for pre and post arc conditions. The arc occurs at the peak potential.

effects can cause significant departure from classic hydrodynamics [11]. To better illustrate the differences between the RM instability and the MHD-RM instability, Fig. 1.6 shows a simple schematic of their evolutions through time. Figure 1.6 A) shows the traditional RM instability with a cylindrical interface. Here a pressure gradient, shown as a shock wave, travels through a shock tube domain and eventually transits the interface. This interface provides the density gradient and is either another species with a different density or the same species at a different thermodynamic state. Typically, the shock wave deposits vorticity on the interface, then travels past the interface and down the domain while the interface moves at the post shock velocity and eventually transitions to turbulent mixing due to the vorticity deposition. However, this is not always the case in MHD.

The MHD-RM instability can show significant suppression of mixing [47, 48, 49]. This suppression can be attributed to waves, which exist in MHD but not in classic hydrodynamics, that work to remove vorticity from the interface. These waves are 
known to be both hydromagnetic and magnetosonic waves [50]; the effects of which can be seen in Fig. 1.6 B). In this figure waves form at the interface due to the presence of the magnetic field. As vorticity is deposited along the interface, a velocity gradient appears with components both perpendicular and parallel to the interface. These disturbances, or velocity components, are then propagated away from the interface due to the magnetic field as either Alfven waves, for the velocity along the magnetic field, or magnetosonic waves, for the velocity perpendicular to the magnetic field [51].

It is from the interaction of the motion of charge, either parallel or perpendicular to the magnetic field, that gives rise to the MHD waves. In fact, the MHD wave dispersion equations can be easily derived for either orientation [51]. While the derivation is not shown here, the dispersion relations are used to find the velocity at which the MHD waves propagate. A MHD wave will propagate perpendicular to a magnetic field at the Alfven velocity, given as $v_{A}=B / \sqrt{\mu_{0} \rho}$. This wave is known as the hydromagnetic wave and is a function of the magnetic field strength. Similarly, the MHD wave which propagates along a magnetic field line is called the magnetosonic wave, which can be calculated as Eq. 1.16. The velocity of this wave is in terms of the phase velocity, $\Omega / k$. This wave is sometimes called a fast hydromagnetic wave as it can be written as a function of the Alfven velocity, $v_{A}$. The magnetosonic velocity is also a function of the speed of light and the acoustic velocity or $c$, and $v_{s}$. It's important to note that the magnetosonic velocity will tend towards the acoustic velocity as $B$ goes to 0 . It is the hydromagnetic waves which suppress the RM instability in MHD by removing the vorticity from the interface. This is because the vortical motion acts to disturb the magnetic field and the magnetic tension force which arises, Eq. 1.14, acts to restore the field at the Alfven velocity. 


$$
\begin{gathered}
\vec{J} \times \vec{B}=\frac{(\vec{B} \cdot \nabla) \vec{B}}{\mu_{0}}-\nabla \frac{B^{2}}{2 \mu_{0}} \\
\nabla \cdot \vec{B}=0 \\
\frac{\Omega}{k}=\sqrt{c^{2} \frac{v_{s}^{2}+v_{A}^{2}}{c^{2}+v_{A}^{2}}}
\end{gathered}
$$

Thus, in the presence of a sufficiently strong magnetic field the RM instability in MHD is suppressed as the mechanism of growth is no longer able to act on the interface. Figure 1.6B) shows a magnetic field orientation perpendicular to the shock transit. For any field orientation both magnetosonic and Alfven waves will occur, acting to stretch and remove the vorticity from the interface, though the degree of and mechanisms for damping the RM instability change with orientation of the field to the shock direction.

Similarly, one can find the dispersion relation for these waves when considering the ideal MHD equations. The transition from classic hydrodynamics to MHD demands additions to the equations used to solve these systems. These ideal MHD equations are shown as Eqs. 1.17 through 1.20 including Eqs. 1.14 and 1.15 and are the continuity, momentum, energy, amperes law, and Gauss's law of magnetism respectively. While the continuity equation, Eq. 1.10, remains unchanged; the other equations are either modified or complete additions to the system of equations.

The momentum equation, Eq. 1.18, has the addition of the electromagnetic forces which are represented by the cross product of the current density, shown as $J$, and the magnetic field, $B$, over the speed of light, $c$. This term is the Lorentz force without an externally applied electric field. Amperes law expands the Lorentz force in as Eq. 


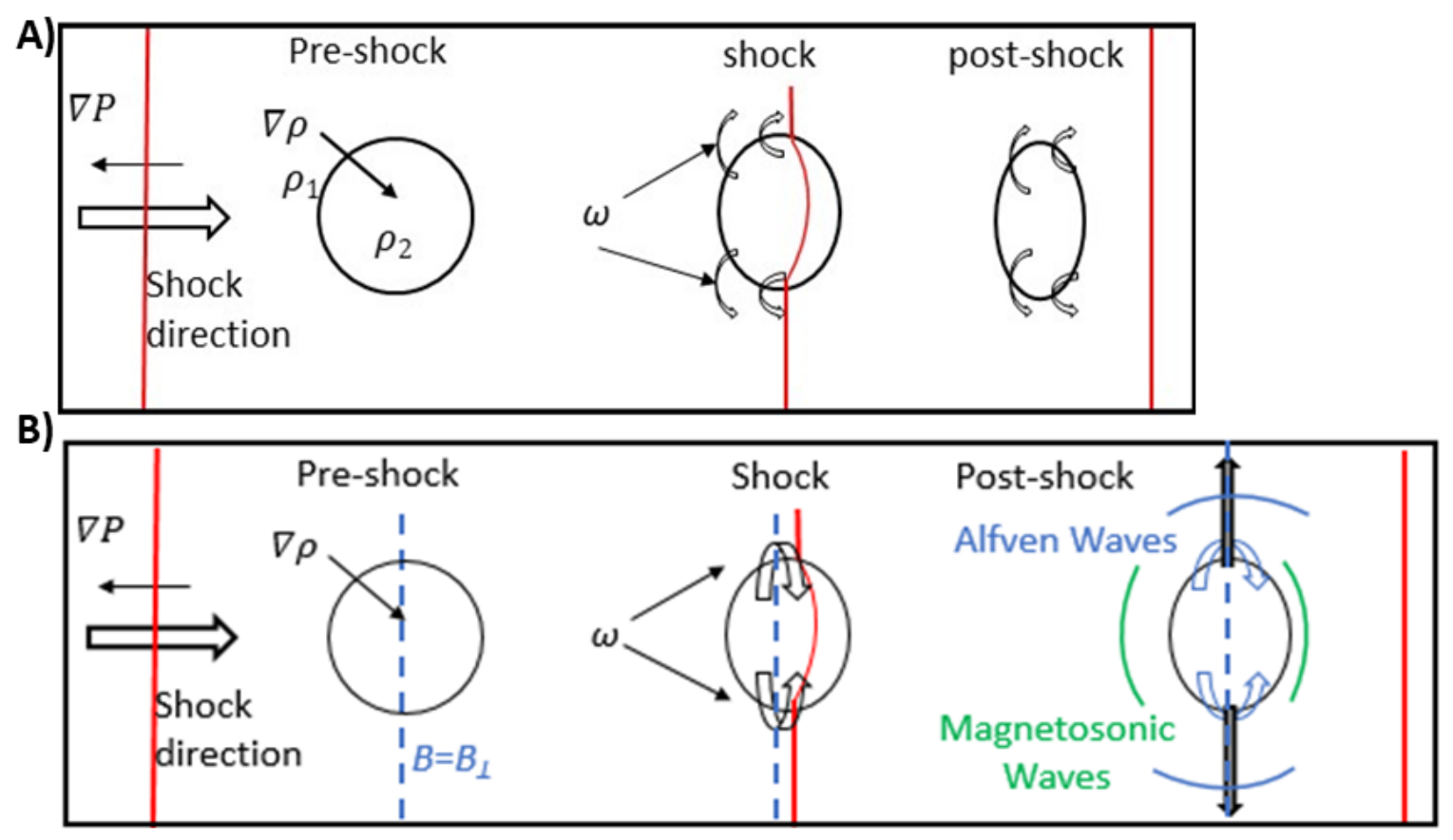

Figure 1.6: 2D schematic of the cylindrical Richtmyer-Meshkov Instability driven by a shock wave in A) traditional hydrodynamics and B) with magnetohydrodynamic effects.

1.20 to give Eq. 1.14. This form of the Lorentz force has a right-hand side whose first term gives the magnetic tension force, that acts to straighten any disturbed magnetic field lines giving rise to the MHD waves, and the magnetic pressure. Equation 1.19 contains $e$, which is the total energy per unit volume.

$$
\begin{gathered}
\frac{\partial \rho}{\partial t}-\nabla(\rho \vec{u})=0 \\
\rho\left(\frac{\partial}{\partial t}+\vec{u} \cdot \nabla\right) \vec{u}=\frac{1}{c} \vec{J} \times \vec{B}-\nabla P \\
\frac{\partial e}{\partial t}+\nabla\left[\left(e+P+\frac{B^{2}}{2}\right) \vec{u}-\vec{B} \vec{u} \vec{B}\right]=0
\end{gathered}
$$




$$
\vec{J}=\frac{c}{4 \pi} \nabla \times \vec{B}
$$

In consideration of the research done in literature previously, and with future hydrodynamic experiments in mind, Chapter 2 will present the creation of an atmospheric plasma torch for use as an interface for the RM instability in MHD. Chapter 3 will explore a cylindrical interface of perfectly conducting gases in the presence of a magnetic field through simulations. The simulations presented are 2D in cartesian coordinates and consider a 2D $(\mathrm{R}-\Theta)$ slice of a cylinder interface with a planar shock wave. In Chapter 3, instead of referring to the interface as a shocked-bubble the nomenclature of cylindrical will be adopted with consideration for previous RMI literature on both experiments and simulations. Chapter 4 will expand upon this work to $3 \mathrm{D}$ to study the RM instability with MHD effects. The nature of the cylinder is such that it provides a double interface $[18,19,20]$ and does not have a preferential direction with respect to the shock wave or the magnetic field; thus the cylindrical interface was chosen for its unique geometry. However, the growth of the cylindrical interface exhibits a dependence on the orientation of the magnetic field relative to the shock wave. In Fig. 1.7, the shock wave is shown traveling in the $Y$ direction. This means the site of maximum vorticity deposition is $|\alpha|=\pi / 2$. However, if the shock wave direction was changed such that it was traveling in the $X$ direction the maximum vorticity would occur on $|\alpha|=0$, or orthogonal to the shock wave direction. Assuming the interface is allowed to evolve uninterrupted by boundaries and magnetic fields the evolution of the cylindrical RM instability would be the same between these two cases.

However, in the presence of a magnetic field the evolution becomes suppressed and MHD waves work to carry the vorticity away in the direction of the magnetic 
field. If both the shock wave and magnetic fields are aligned, the Alfven waves work to transport the vorticity away in the direction of the flow (shock transit direction). This is shown in Fig. $1.7 \mathrm{~A}$ ), with the vorticity being stretched in $Y$, the direction of the shock wave motion. Similarly, Fig. $1.7 \mathrm{~B}$ ) presents a scenario where the shock wave is traveling in $Y$ and the magnetic field is in $X$, or perpendicular to the shock wave direction. Here the vorticity is carried perpendicularly away from and through the interface. Thus for other perturbations, such as the classically studied sine wave, one may be interested in transverse or normal field orientation relative to the interface; for the cylindrical interface it does not make sense to discuss normal and transverse directions to the interface but rather the orientation of the shock wave (pressure field) to the magnetic field. In this way the cylindrical interface is unique as it is independent of the direction of the shock wave and is only sensitive to the orientation of the fields relative to each other. While work has been done on a converging cylindrical and spherical interface, both the density and pressure gradients shared the radial geometry $[52,53]$. In addition to the interesting properties of the cylindrical interface with respect to the hydrodynamic and MHD effects, the cylindrical interface is also relatively simple to make for a plasma, as will be shown in Chapter 2.

\subsubsection{Literature Review for Plasma and Magnetohydrody- namics}

Magnetohydrodynamic instabilities involve the coupling of hydrodynamic instabilities with magnetic field effects in a conducting fluid. While experiments have been done which may include the MHD instabilities, these experiments were done in large-scale facilities (e.g. MagLIF [41, 42] and OMEGA [40] experiments) and were not neces- 


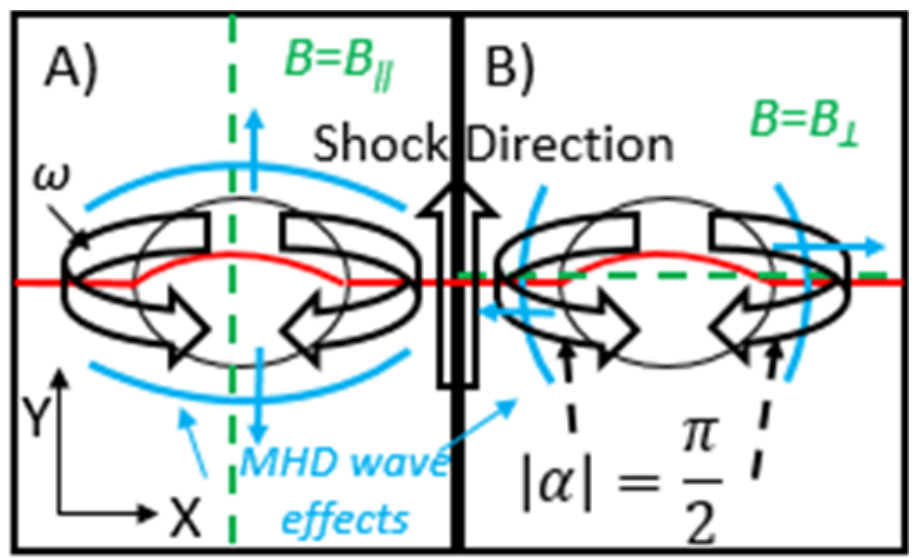

Figure 1.7: A 2D schematic representing the effects of the magnetic field orientation relative to the shock wave direction on vorticity. A) The magnetic field is parallel to the shock wave direction cause vortex translation parallel to the shock wave. B) The magnetic field is now perpendicular to the shock wave causing vortex translation transverse to the shock wave. In both magnetic field orientations, the site of maximum baroclinic vorticity deposition is $|\alpha|=p i / 2$.

sarily done to study the interaction of instabilities with MHD. However, considering the similarities between hydrodynamics and MHD, one should consider the types of experiments which could provide interfaces that could be considered in MHD. One approach to study these effects in a lower energy environment so is to impulsively accelerate (shock) an atmospheric-pressure plasma cylindrical interface (i.e. a plasma torch) using a shock tube facility with an applied strong external magnetic field. Thus this section will provide background on the atmospheric plasma sources which could be utilized in such experiments as well as simulations which have considered MHD effects on similar systems.

Since the mid-1950's, plasma torches have been studied for a wide variety of applications, including material processing for surface modification, biomedical treatment for wound healing and sterilization, metallurgy, metal cutting, waste management, laser and space technology, and scientific research of plasma chemistry and physics [46]. As such there exists extensive research on plasma torch devices, their design, 
and operation. Many of their applications require atmospheric operation, in which it is more difficult to ionize gases due to the substantial increase in required breakdown voltage. Previous studies have focused on the development and characterization of laminar atmospheric-pressure plasma torches (APPTs) and focused on optimizing voltage-current relationships, gas flow rates, and torch design parameters [54]. Understanding the effects of these parameters is imperative when developing APPT. Comparison of plasma characteristics may be evaluated using similarity theory, in which one experimental property is used to determine the effect of others without physical experimentation $[55,56]$. Plasma jet velocity measurements have been made using particle imaging methods by injecting particles into the plasma in order to understand plasma flow conditions $[57,58]$.

In addition to understanding the flow conditions, it's important to characterize the plasmas inherent electric properties. The optimal method often depends on the plasma application. For instance many low pressure plasmas utilize the Langmuir probe technique. In this technique a wire is held at some biased potential, represented by $V_{B}$, and is either inserted into the plasma $[59,51]$ or swept through the plasma [60]. The potential difference between the plasma and the probe generates a current, $i_{p}$ which is then recorded via a connected data acquisition system (DAQ), a simple schematic is shown in Fig. 1.8. This process is repeated as the probe potential is changed between some negative range to some positive range; often limited by the DC power supply used to bias the wire. As the potential is more negative, more ions will be drawn to the probe and vise versa. The $V_{B}$ range for the potential is application based, but can be determined by the ion and electron saturation currents, or $I_{i s}$ and $I_{e s}$ respectively. These are found by adjusting the potential on the probe more negative/positive until the current drawn due to the potential difference stops increasing/decreasing. Once this range is found a figure like the one shown in Fig. 


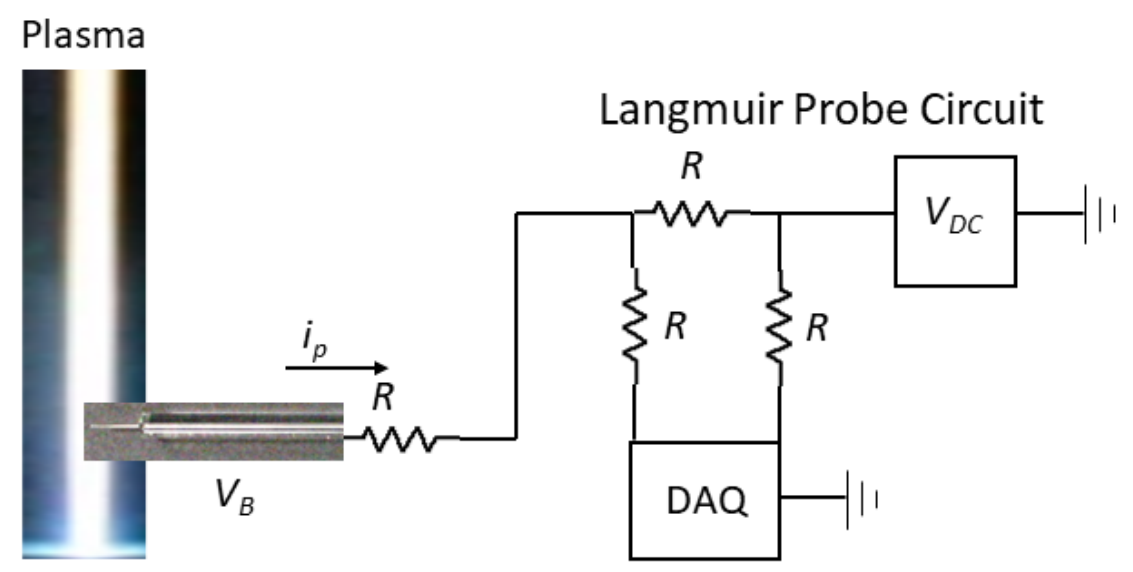

Figure 1.8: Single Langmuir probe schematic: A metal wire probe is insulated in capillary glass, biased to some known $\mathrm{DC}$ voltage, $V_{B}$, and then inserted into the plasma. The potential difference between the plasma and the probe drives current into the probe, $i_{p}$, which is read by the DAQ. Resistors are used to help control the voltage at the measurement points as needed. This process is repeated to acquire data on the electric properties of the plasma

1.9 can be generated and the floating potential, $V_{f}$, and the electron temperature, $T_{e}$, can be found. The floating potential, or the potential of the probe which draws 0 current, and the plasma potential $V_{p}$, the potential of the plasma itself, may seem as though they should be equal; however when the probe is inserted into the plasma at some potential a sheath forms which serves to change the local spacial electric field. The charge within this sheath then must be overcome to find the plasma potential, shown to occur near an inflection point in Fig. 1.9. For static or contained plasmas a stationary probe may be used. However, for flowing plasmas or plasmas whose electric characteristics may vary spatially sweeping probes may be beneficial [60].

Using the parameters gathered by the Langmui probe one may also calculate the electron temperature. From the electron temperature, and the assumption that the electron and ion temperatures are similar, one can calculate the bulk temperature of the plasma. This is advantageous for simulations for assigning the thermodynamic state of the plasma. To calculate the electron temperature from the Langmuir data 


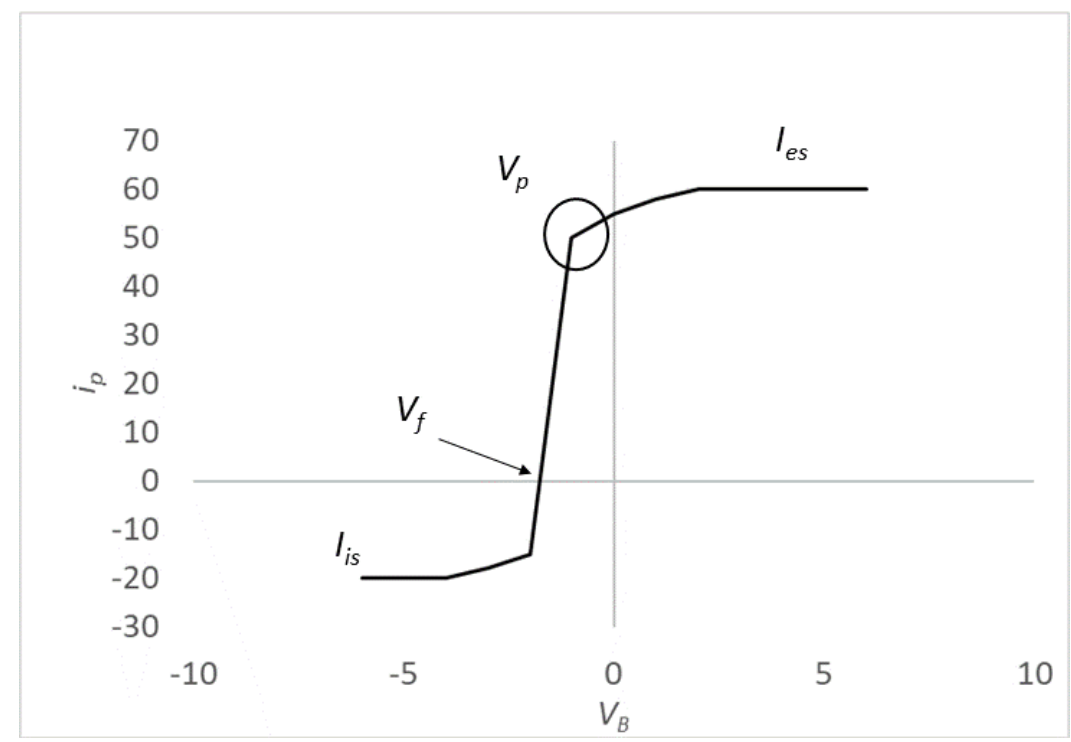

Figure 1.9: Idealized Langmuir probe data. Here one can see how the various properties can be extracted directly from the probe.

first one must subtract the ion saturation current everywhere. After this, take the natural log of the probe current and find the slope of the most linear region (neglecting the region past the plasma potential inflection). This slope is an estimate of the electron temperature [61]. Using this value in the boltzmann equation, Eq. 1.21 can help determine the number of charged species in the plasma, which in turn can be used in the Saha equation [51], Eq. 1.22 to determine bulk plasma temperature. The Boltzman relation gives the number density of the charged species, given as $n$ where the subscript $j$ is meant to represent either ions or electrons, as a function the total number density, the charge of the species, the potential of the species, the boltzmann constant, and the temperature of the charged species, or $n_{0}, q_{j}, \theta, K$, and $T_{j}$ respectively. The Saha equation then uses the number densities and the ionization energy of the gas, $U$, to solve for the bulk temperature of the gas, $T$.

$$
n_{j}=n_{0} \exp \left(\frac{-q_{j} \theta}{K T_{j}}\right)
$$




$$
\frac{n_{e}}{n_{0}}=2.4 \times 10^{21} \frac{T^{3 / 2}}{n_{e}} \exp \left(\frac{-U}{K T}\right)
$$

Despite the effectiveness of the langmuir probe at low pressures there are challenges with high pressure plasmas. First off, the langmuir probe uses a number of assumptions which fall apart at high pressures $[60,62,63]$. For instance, the langmuir probe assumes that the mean free path of the plasma is much greater than the probe radius [59]. While this may hold in low pressure systems, as the particle number density increases the mean free paths shrink significantly while the probe radius remains on the order of millimeters. Another assumption is that the degree of ionization is low, or that the plasma is quasi-nuetral. However, depending on how the plasma is generated it may have very high ioniziation near the electrodes. Despite the challenges, some groups have used langmuir probes for high pressure applications [60, 62, 63]. Langmuir probles can be single probe configurations, like Fig. 1.8 or can be expanded to have double $[64,65,66]$ or triple [67] probes. The additional probes work to eliminate noise from the plasma sheath by causing a smaller current drain and have been used successfully at high pressures.

While electrostatic probes may seem an easy solution, the act of inserting the probe may distrupt both electrical and flow characterstics of the plasma. As such, many noninvasive techniques have been developed to overcome the disadvantages of intrusive probes. These methods include quantitative shadowgraphy, Schlieren imaging, spectroscopic methods, and the atomic Boltzmann method, each of which have been used to calculate various plasma characteristics such as plasma temperature $[68,69,70,71,72]$. Quantitative shadowgraphy and Schlieren imaging have been used to determine the rotational temperatures of gas and visualize the hydrodynamic characteristics of plasma jets [69], [70]. 
Laser scattering methods (LSMs) are another type of nonintrusive optical diagnostic. LSMs have been used on atmospheric-pressure plasmas to investigate the gas temperature using Rayleigh scattering, electron temperature and electron density via Thomson scattering (TS), and molecular densities and rotational temperature by Raman scattering [68]. TS is used also to spatially and temporally resolve the electron density and energy distribution when the gas temperature is a few orders of magnitude less than the electron temperature [73]. Since atmospheric plasmas do not always operate within the laminar regime, plasma turbulence imaging has been performed using TS as well [74].

Significant contributions have been made towards understanding atmosphericpressure plasma through optical emission spectroscopic methods have been made in the last three decades $[75,76,77]$. These studies measured the temperature and composition of single-element or multi-element thermal plasmas by implementation and modification of the Fowler-Milne spectroscopic method using the Abel inversion technique. Although traditionally applied to free-burning arcs, this method proves to be an excellent diagnostic for non-transferred APPTs after some modifications [78, 79]. The spectroscopic determination of radial temperature distribution profiles is one of the most prevalent methods implemented for measuring atmospheric plasmas. Emission spectroscopy is based observing the intensity of emitted light for some spectral distribution of the plasma and is used to recover radial temperature distributions.

The Fowler-Milne spectroscopic technique, and its modifications, can be used to determine both the composition and temperature of multi-element thermal plasmas $[75,76,77,80,81,82]$. Other methods utilizing a monochromator have been performed utilizing emission spectroscopy [83]. These methods, however, can lack the ability to acquire space-time data instantaneously, simultaneously, or to a high degree of accuracy; radial profile temperature distributions traditionally require obtaining 
individual data points as a spectrometer or monochromator is incrementally swept across a plasma. The disadvantage here is low spatial and temporal resolution.

While no MHD-RM instability experiments have been done to date, there have been groups who have begun to study this instability through simulations and theory. Samtaney [47] studied an inclined MHD-RM instability, with a magnetic field parallel to the shock wave direction and compared it to an inclined RM instability without a magnetic field. In this work, it was shown that the magnetic field suppresses RM instability growth and mixing. Wheatley et al. $[48,49,50]$ and Mostert et al. $[52,53]$ continued this work and studied a sinusoidal interface in planar and converging cylindrical/spherical geometries. Across several articles these authors performed a full parametric study to include the effects of magnetic field orientation, increasing $M$, the ratio of $\eta$ to $\lambda$, Atwood number, and non-dimensional magnetic strength. The non-dimensional magnetic strength, shown in Eq. 1.23, represents the ratio of thermodynamic pressure to magnetic pressure and is a function of the magnetic permeability, $\mu_{0}$, and the magnetic field strength, $B$. They found that, while the Mach number and Atwood number effects were similar to those in classic hydrodynamics, by varying the orientation of the magnetic field to be normal or transverse to the interface the RMI in MHD would exhibit different qualitative evolution and suppressed growth rates; and that increasing the magnetic field strength further dampened the instability.

$$
\beta=\frac{2 P \mu_{0}}{B^{2}}
$$




\section{Chapter 2}

\section{The developement and characterization of an atmospheric non-transferred plasma source for magnetohydrodynamic experiments}

The development and diagnosis of an atmospheric pressure, non-transferred, DC arc plasma torch for investigating magnetohydrodynamic experiments is presented within this chapter. Utilizing a custom $50 \mathrm{kV} \mathrm{AC}$ starter circuit coupled with a high power DC power supply a long laminar plasma jet was successfully generated by adjusting voltage and current, gas flow rates, and the torch nozzle diameter and length. To allow for continuous torch operation copper, aluminum, and tungsten alloy electrode components are utilized in conjunction with an electrically isolated water cooling system. The $50 \mathrm{kV}$ AC starter circuit is coupled with a $15 \mathrm{~kW}$ DC power supply. Axial and tangential gas flow, with nitrogen as the working gas, is regulated to generate a stable laminar atmospheric plasma torch with a jet length of over $80 \mathrm{~cm}$ under 
ideal operating conditions. To visualize the plasma interface in MHD experiments a technique was developed to image the cylindrical plasma jet in both the $R$ - $Z$ plane (axial) and $R-\theta$ plane (cross sectional) using planar-laser Mie scattering from particles added to the surrounding gas. Plasma emission wavelengths are filtered out allowing scattered light from the particle laden-flow field illuminated at $532 \mathrm{~nm}$ by an Nd:YAG laser to be isolated. Plasma electron temperature and degree of ionization are estimated using a technique based on the Fowler-Milne spectroscopic method. Electron temperatures of up to $14,000 K$ and degree of ionization up to $27 \%$ at the torch exit were obtained. The classic Langmuir Probe technique was also considered and ultimately decided to have been unsuccessful, though the experiment is detailed here with suggestions and observations on how to improve the diagnostic tool.

\subsection{Developement of an Atmospheric Plasma Torch for Investigating Magnetohydrodynamic Insta- bilities}

Different methods have been implemented to produce atmospheric plasma, such as $\mathrm{RF}$ induction coils, transferred arcs (e.g., free-burning arcs), non-transferred arcs

(e.g., APPT), and plasma arc heaters. However, each of these methods must undergo electric breakdown to generate a plasma l[46]. Because the plasma torch developed for this work was designed to be implemented inside a shock tube, the plasma containing region must be sustained in an unobstructed atmospheric environment and is desired to possess a minimum length of $14.5 \mathrm{~cm}$, the height of the internal cross section of the Missouri Shock Tube [84]. Since non-transferred arc plasma torches provide the largest free plasma jet length this method was selected for our device.

In addition to the jet length, the operating conditions of the device are incredibly 
important for the planned hydrodynamic experiments. For example, many different gases and mixtures of gases have been utilized in the study of atmospheric pressure plasmas. In particular argon, air, helium, and oxygen are commonly used. For our purposes, nitrogen is ideal due to its ionized physical properties. Its limited and ideally located emission lines enable convenient diagnostics (discussed later in more detail in section 2.2.2). For nitrogen, a breakdown potential upwards of $10 \mathrm{kV}$ is required at atmospheric pressure with an electrode spacing of $2 \mathrm{~mm}$. This electrode spacing is radially, allowing for a jet diameter of $4 \mathrm{~mm}$ at the exit of the torch. This diameter was deemed acceptable for use in future shock tube experiments.

The V-I characteristics of the torch must also be considered in its design. Many studies have been performed on atmospheric pressure plasma torches specific to the voltage-current characteristic of the arc. Pan et al. examined the V-I characteristics of a laminar argon DC APPT utilizing arc voltages of 33-50 V, and arc currents of 75-220 A [85]. Planche et al. implemented arc currents from 200-600 A with arc voltages of 50-80 V to investigate plasma jet velocities [57]. Das et al. measured V-I characteristics of DC arc plasma utilizing arc voltages of 20-50 V and arc currents of 150-400 A [86]. It is known that as arc current increases, the arc voltage and plasma jet length also increase. Thus, an arc voltage of up to $100 \mathrm{~V}$, an arc current of up to 200 A was chosen to develop a laminar plasma jet with sufficient length to be implemented within the FMSTL shock tube for future investigation of the MHD RM instability.

Of equal importance to the V-I characteristics, the gas flow rates also govern the length and stability of the plasma jet. Both axial and tangential gas flows are used in the torch to achieve laminar flow operating conditions. The tangential gas flow rotates the arc attachment around the nozzle to ensure even erosion of the anode. Previous works used gas axial flow rates in the range of 5-27 SLM for generating a 
long laminar atmospheric pressure plasma jet [85, 87]. Digital mass flow regulators were used in this work to vary the ratio of axial and tangential gas flow as well as the total gas flow rate supplied to the torch.

\subsubsection{Atmospheric-Pressure Plasma Torch Design}

Although much research has been published on APPTs, the process of coupling a high voltage starter circuit necessary to meet the breakdown voltage and initiate a spark with a high current DC power source, needed to sustain the arc, is seldom discussed. This chapter will similarly neglect this. This was done for two reasons. One, this was not the focus of the work done by this author for the completion of their doctoral degree but rather by their colleague, and two the publication this chapter is based on has a detailed section covering these details. Instead this chapter will focus on work contributed by the author and that is pertinent to MHD RM instabilities. That said, the creation of the APPT necessitated the developement of an AC starter circuit which could be paired with a DC power supply. The AC circuit served to provide the high voltage required to break down the working gas. After a discharge was formed, the DC power supply was activated and the AC supply shut off. This allowed the DC supply to sustain the discharge at a much lower voltage and mitigated any fluctations that would be introduced by the AC signal.

To ensure the DC power supply would deliver 200 A to the torch during operation, water cooled power resistors (WCPRs) were implemented in series with the torch electrodes. The WCPRs were selected and wired in parallel such that they were capable of handling a $200 \mathrm{~A}$ current with an equivalent total resistance of $0.23 \Omega$. The WCPRs stabilized the V-I requirements of the torch during the spark-to-arc transition where there is a sharp, instantaneous, decrease in voltage that is beyond 
the slew rate (measurement rate) of the power supply. Without this components, the decrease in voltage would result in a low DC potential and failure of the spark-to-arc transition. For more information, please see [2].

When designing the plasma torch careful consideration was given to safety, repeatability, and efficiency. The major components of the torch are split into two sections, the anode (top) section and the cathode (bottom) section. The two sections were designed to be electrically isolated using alumina insulating disks. This ensured that electric breakdown occurred between the anode and cathode in the arc chamber section rather than spuriously occurring between assembly components. As a point of safety, the entirety of the cathode section is housed inside the anode section, which is operated at the ground potential, such that during operation, only the ground potential was physically accessible. Figure 2.1(A) shows a cross section of the torch design.

Material properties are important to arc generation and specific materials are desired for the anode and cathode components. The use of materials with low boiling points and thus higher current densities will aid in the transmission of electrons through the arc as the anode and cathode sections are heated. Additionally, materials with higher electrical resistivity result in faster material erosion rates. This can be counteracted by high gas flow rates which decrease cathode erosion. A thoriated tungsten rod was used for our cathode and our anode comprised of Copper 182 alloy. The remaining components of the torch body (not in contact with the arc) were fabricated from 6061-T6 aluminum.

Modular sections were used in the torch to allow for efficient design modifications and repair. To optimize the plasma jet length the modular design was leveraged to test different nozzle diameters, ranging from 4-10 mm, and lengths, from 15-80 mm. Additionally, the copper alloy sections were designed as press fit inserts to the top 


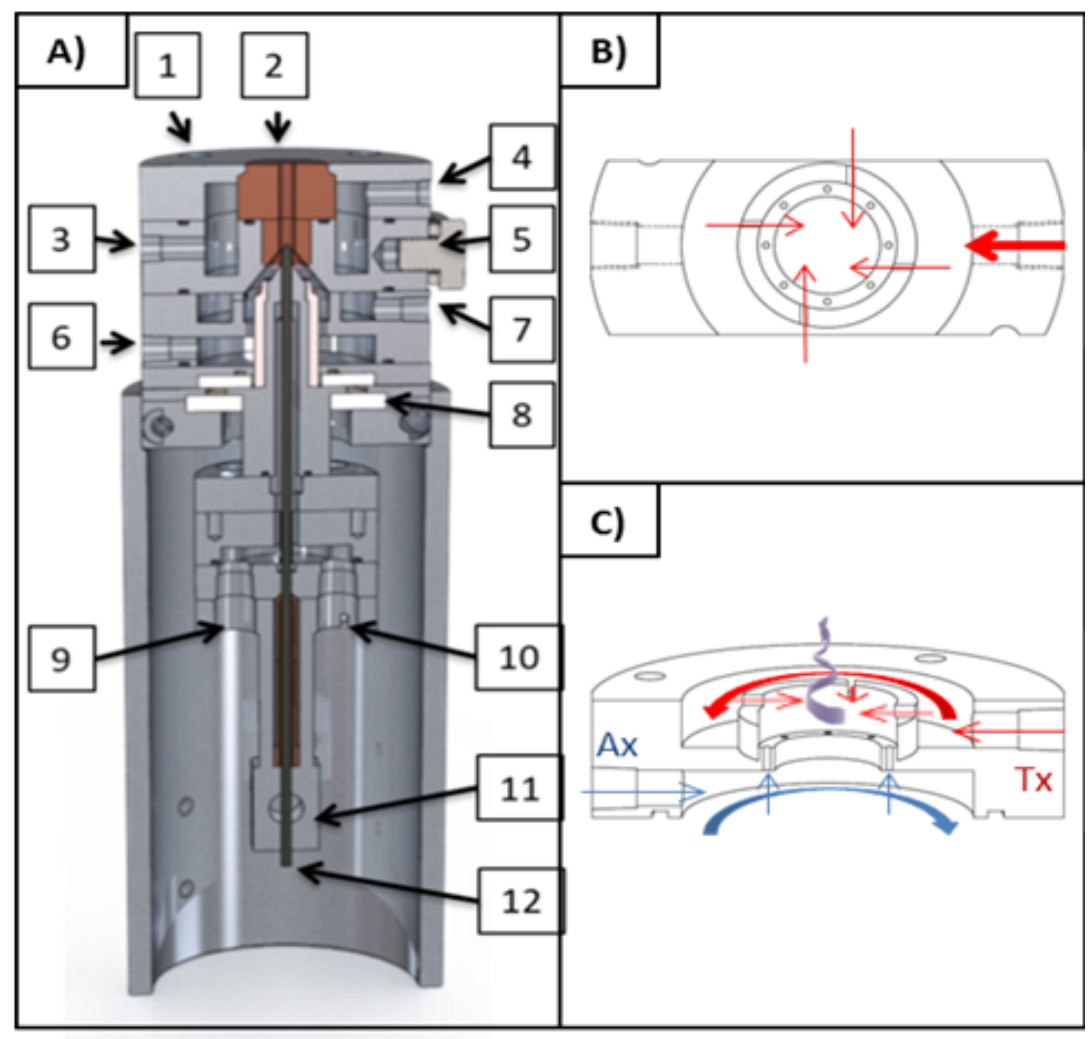

Figure 2.1: (A) APPT cut-out diagram 1) anode, 2) nozzle, 3) anode water cooling inlet, 4) anode water cooling outlet, 5) High current DC in $(+), 6)$ axial gas (blue) inlet, 7) tangential gas (red) inlet, 8) alumina washers, 9) cathode water cooling inlet, 10) cathode water cooling outlet, 11) High current DC out (-), 12) thoriated tungsten cathode. (B) Top view of axial and tangential gas flow component. (C) Isometric cutout representation of axial (blue) and tangential (red) gas flow paths. 
and bottom sections of the anode. Thus, the anode could be replaced without having to replace the entirety of the anode section ensuring repeatable operation as erosion occurred.

Given the large amount of heat transfer that occurs from the plasma to the anode and cathode components, it is impossible to operate the torch for long periods of time, given material limitations (i.e., melting temperature), without an active cooling system. This active cooling is achieved in our torch by water cooling loops within both sections. Since the anode section is driven to a ground potential a once-through cooling circuit is used where tap water is filtered, flowed through the section and dumped to a drain. The cathode section, at some positive DC voltage, requires a more sophisticated cooling approach. An electrically isolated, closed-loop deionized water cooling system, due to its lower than tap water electrical conductivity, is used to ensure that the electrical potential of the cathode is not transmitted through the water cooling loop, (i.e., outside the cathode section). The selection of nitrogen as the working gas also provides additional cooling capacity, as diatomic nitrogen has a higher specific heat than many other commonly used gases (e.g., argon). Previous works utilized water cooling flow rates of 10-20 SLM while observing plasma torch electrical characteristics $[55,86]$. Valincius et al. investigated thermal characteristics of a plasma torch finding that the temperature rise of the cathode was much smaller than that of the anode [88].

A final torch design was selected with a nozzle length of $15 \mathrm{~mm}$ and diameter of $4 \mathrm{~mm}$. Various gas (tangential and axial) flow rates and arc currents were tested to achieve the maximum plasma jet length. Axial gas is injected into the anode section and flows upwards around the cathode section through flow ports which are parallel to the nozzle central axis. Tangential gas flow is also injected in the anode section through axially offset flow ports, perpendicular to the nozzle central axis, where it 
mixes with the axial gas from below. The axial gas provides the majority of the working gas to be ionize and its flow rate is the main contributor in determining the stability of the plasma. The tangential gas subsequently aids in even distribution of anode erosion and ablation effects from the arc attachment within the nozzle. This can be visualized in Fig. 2.1 (B) and (C). A regiment of diagnostic techniques, discussed later, were used to quantify the effect on the plasma jet length and ionized region length. Additional measurements were performed to estimate the degree of ionization and plasma temperature at the optimized flow conditions.

\subsection{Characterization of an Atmospheric Plasma Torch for Investigating Magnetohydrodynamic Insta- bilities}

In addition to creating the plasma source, diagnostics were also explored. This diagnostics were created so that the electric and flow properties of the plasma could be measured and qualified. The electric properties are useful in helping future experimentalists to understand how the plasma might be effected by external magnetic fields during instability experiments. The flow properties similarly are important in understanding the initial conditions to the hydrodynamic experiments. Two methods used are used to analyse the electric properties of the plasma: the invasive Langmuir probe technique and the spectroscopic based Fowler-Milne technique which is modifed via the Abel inversion technique. This will be discussed more in section 2.2.1. The spectroscopic method is also briefly discussed. More details can be found in [2]. Two qualitative measurements were also used to try to understand the plasma jet shape as an interface for hydrodynamic experiments. The plasma emissions were first imaged without a filter in order to capture the height and radius of the visible emissions. 
However, it becomes difficult to get exact measurements due to light saturation and so filtering must occur for exact plasma diameters (in $R-\theta$ ) and thickness/heights (in $R-Z$ ). The particle scattering-Mie method was combined with an optical filter used to filter out emissions to capture qualitative images of the plasma interface. This is useful for hydrodynamic experiments and for determining how the interface might evolve post-shock in experiments or for prescribing the interface in simulation space.

\subsubsection{The Langmuir Probe}

Enthalpy probes are among the earliest diagnostics used in plasma experiments. Historically, a probe was placed within a plasma volume and the current induced between the plasma at some voltage, $V_{f}$, and the grounded probe would be recorded. It was thought that this was the plasmas potential, but as the technique developed it was later discovered that this was the floating potential [51]. Langmuir then developed the technique further by biasing the probe to some known voltage, $V_{B}$, allowing experimentalists to gather data and calculate electron temperatures, number densities, and the plasma V-I characteristics. While much of this work was done for low pressure plasmas, some groups have modified the langmuir probe for high pressure applications.

To explore the plasma V-I characteristics a langmuir probe circuit was developed, Fig. 2.2. This circuit utilized several resistors to step down the voltage as it was read by a DAQ (LabJack U6 DAQ). A DC Power supply (CSII12001X from Circuit Specialists), used to bias the probe had an output of $\pm 120 \mathrm{~V}$ with a max current output of $1 \mathrm{~A}$. Tungsten wire with $0.256 \mathrm{~mm}$ diameter was used for the probe and sheathed in capillary glass. Approximately $1 \mathrm{~mm}$ of tungsten was exposed to act as the probe surface while the capillary glass was used both to shield the remaining tungesten 

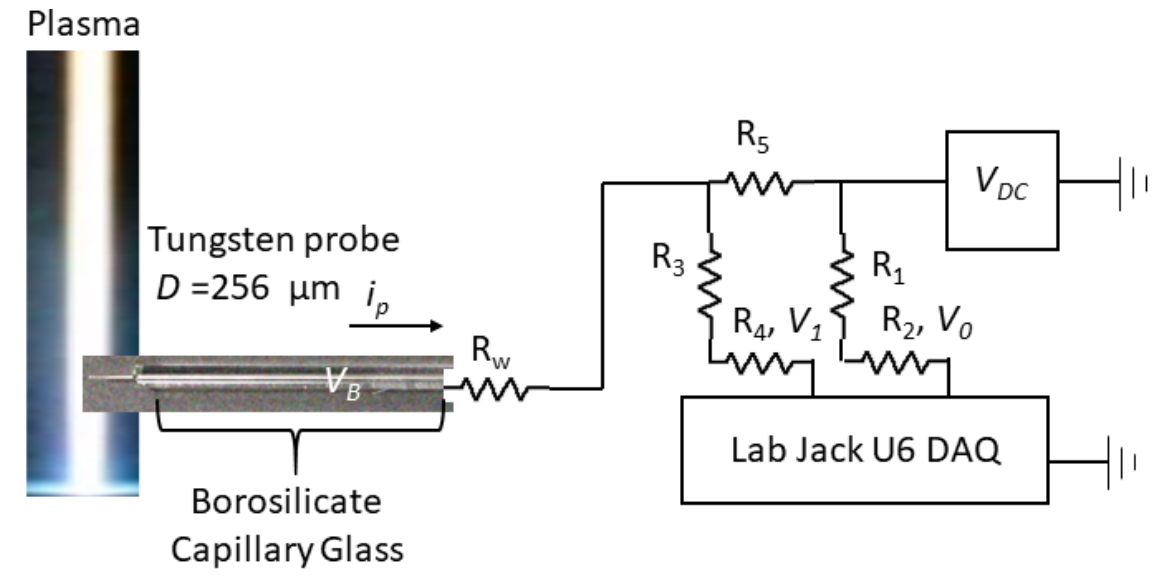

Figure 2.2: Langmuir circuit diagram. Here the tungsten probe with boroscilicate capillaruy glass is placed inside the plasma. The probe, set at some biased potential from the DC power supply, induces a current from the potential difference. This induced current is read by the LabJack U6 DAQ at the potential difference across $R_{5}$. The voltage dividers comprised of $R_{3}, R_{4}$ and $R_{1}, R_{2}$ are used to step down the voltage for acceptable levels for the DAQ

Table 2.1: Langmuir Circuit Operating Parameters

\begin{tabular}{l|r}
\hline DC Voltage: & $\pm 120 \mathrm{~V}$ \\
$R_{1} \approx R_{3}:$ & $\sim 1000 \Omega$ \\
$R_{2} \approx R_{4}:$ & $\sim 81 \Omega$ \\
$R_{5}:$ & $\sim 5 \Omega$ \\
$R_{w}:$ & $\sim 4 \Omega$ \\
\hline
\end{tabular}

wire from developing local charge sheiths which could disrupt the measurement and to help thermally protect the wire due to its low thermal conductivity. Tungsten was selected for it high melting point, $\sim 3700 \mathrm{~K}$. Table 2.1 shows the set resistor values as well as the wire resistance, $R_{w}$, and the power supply range. The capillary glass is commonly used in literature for the shielding purposes. The glass tube used in these experiments is borosilicate with an outer diameter of $2.034 \mathrm{~mm}$ and an inner diameter of $0.28-0.286 \mathrm{~mm}$. The melting temperature of borosilicate is $\sim 1100 \mathrm{~K}$.

The langmuir probe experiments were run with a sweeping configuration. This 
allowed the probe to move through the plasma in order to collect charge data in the $\mathrm{X}-\mathrm{Y}$ plane (corresponding to $R-\theta$ ). To accomplish this the probe was attached to a series of stepper motors which allowed computer controlled motion in X,Y, and Z. The $\mathrm{Z}$ motor would be fixed per run to allow for consistent measurements at some fixed torch height. The probe would sweep through the plasma with a velocity of $50 \mathrm{~mm} / \mathrm{s}$ to partially prevent the tungest from being consumed as well as to acquire data across the diameter of the torch. This velocity was found to be sufficient to prevent notable tungsten consumption, and any slower velocity resulted in complete obliteration of the thin tungesten wire. The data was acquired at a $12.5 \mathrm{kHz}$ frequency, allowing approximately 1000 measurements across the $4 \mathrm{~mm}$ jet diameter. The results from these experiments are shown in section 2.3.2.

\subsubsection{Spectral Methods}

To our knowledge, none of the methods used to diagnose and characterize an atmospheric pressure plasma torch are able to provide instantaneous $2 \mathrm{D}$ measurements of temperature and degree of ionization simultaneously. A method to do so, referred to as the 2D Monochromatic Imagery Method (2DMIM), was developed in [2]. The 2DMIM is based off the Fowler-Milne spectroscopic method and determines the radial temperature distrubution by measuring the line emission intensity produced by the plasma at a known axial height.

Traditionally, intensity measurements are taken at specific spatial locations by spectrometers by scanning through a range of wavelengths. Additional points along the radial direction of the plasma are chosen and the process repeated to formulate a 1D intensity plot. Alternatively, the intensity at a specific wavelength can be measured by a monochromator as it spatially scans across one dimension of a plasma. 
Both methods lack the ability to be either highly spatially resolved, temporally resolved, or both. This is the foundation that the 2DMIM is built upon. By utilizing a high resolution CCD camera affixed with a narrow bandpass filter to image a single emission line of the torch, an instantaneous 2D intensity measurement is acquired. The 2DMIM allows for a precise depiction of the plasma flow field, with each pixel of data corresponding to the intensity of radiated light emitted from the torch both axially and radially.

For spectroscopic analysis of a single species gas the total line emission coefficeint of a specific wavelength must be determined. This coefficient varies with wavelength, thus having some apriori knowledge about what wavelengths may be emitted is necessary. The total line emission can be calculated from Eq. 2.1. Here it can be seen that the total line emission coefficient, $\epsilon_{n m}$, is inversly proportional to the wavelength, $\lambda_{n} m$, of the emitted radition of species $j$ at a specific temperature $T$. This equation represents a transition of electrons from upper energy level $m$ to a lower level $n$, where $h$, $c, g_{m}, A_{n} m, n_{j}, Q_{j}, E_{m}$ and $k_{B}$ are Planck's constant, the speed of light in a vacuum, the statistical weight of the upper energy level $m$, the spontaneous emission transitional probability from the upper to lower energy level, the atomic partition function of species $j$ [89], the excitation energy of upper level $m$, and Boltzmann's constant respectively. $\epsilon_{n m}$ is calculated over a range of temperatures and normalized such that the maximum emission coefficient is 1 . The temperature this occurs at is deemed the "normal temperature". This assumes that the plasma is in local thermodynamic equilibrium (LTE) and has a maximum well defined temperature corresponding to a peak emission coefficient.

$$
\epsilon_{n m}=\frac{h c}{4 \pi \lambda_{n m}} g_{m} A_{n m} \frac{n_{j}(T)}{Q_{j}(T)} \exp \left(\frac{-E_{m}}{k_{B} T}\right)
$$


To use 2DMIM a measurement of the plasmas emitted intensity at the same specific wavelength as the total line emission coefficient is also necessary. To get this, a $2 \mathrm{D}$ chord-integrated intensity profile is experimentally measured in the $\mathrm{X}-\mathrm{Y}$ plane using a CCD camera at some height Z. This profile is used to determine the normalized emission coefficient in the R-Z plane by performing an Abel inversion. In this process, the measured chord-integrated intensity (X-Y plane) is transformed into the radial distribution function of the emission coefficient. This process assumes that the plasma is optically thin, rotationally symmetric, and the emitted radiation is isotropic; thus, no absorption within the plasma occurs. Abel inversion recovers three-dimensional data from the measured chord-integrated two-dimensional data.

The chord integrated intensity, $I(x)$ must be expressed as a continuous function and is given by Eq. 2.2, which is then analytically inverted to obtain the emission coefficient given by Eq. 2.3. Similarly to the calculation involving Eq. 2.1, the emission coefficient from 2.3 is normalized. This data exists per pixel per row of data received from the CCD image. The $2 \mathrm{DMIM}$ is then used to recover a $2 \mathrm{D} \mathrm{R}-\mathrm{Z}$ profile of the emission from the APPT by incorporating Abel inversion through generation of an upper triangular area matrix constructed by geometric relations developed in literature $[90,2]$. With the normalized radial distribution of the emission coefficient per row per pixel and the total emission coefficient vs. temperature profile calcualted, 2D spectroscopic measurements can be made.

$$
\begin{gathered}
I(x)=2 \int_{x}^{R} \frac{\epsilon(r) r}{\sqrt{\left(x^{2}-r^{2}\right)}} d x \\
\epsilon(r)=-\frac{1}{\pi} \int_{r}^{R} \frac{I^{\prime}(x)}{\sqrt{\left(x^{2}-r^{2}\right)}} d x
\end{gathered}
$$

To determine the best wavelength to use for the 2DMIM, the emission line spectral 


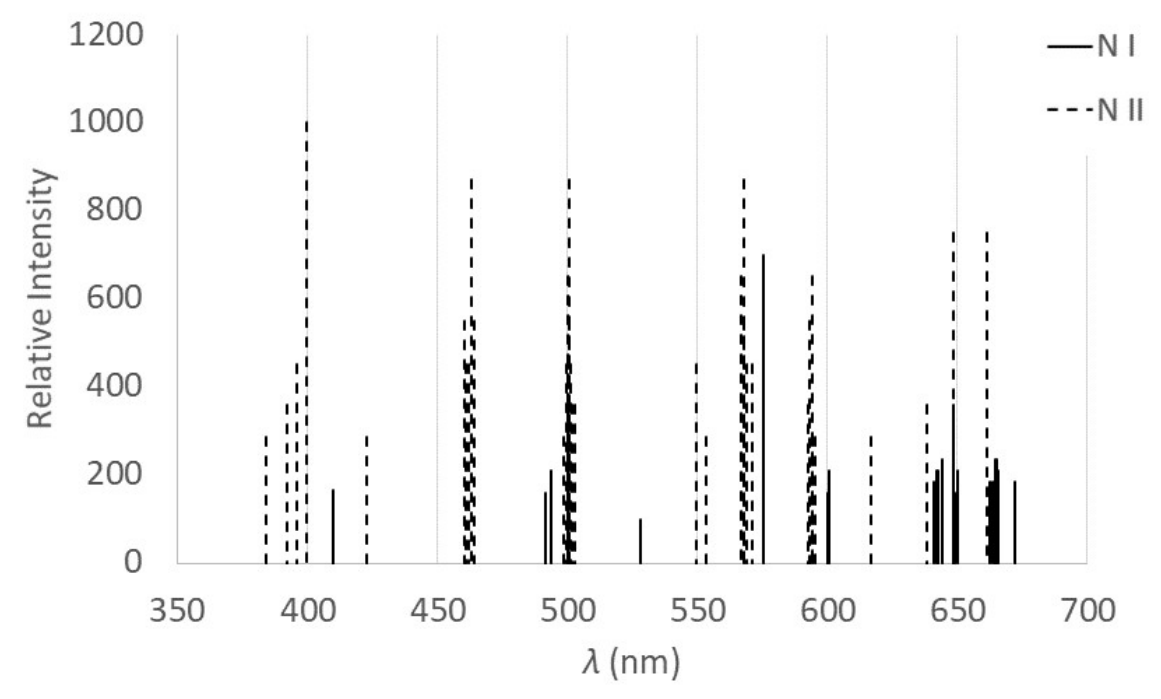

Figure 2.3: Dissociated Nitrogen (N-I) and singly ionized Nitrogen (N-II) emission wavelengths vs intensity

distribution intensity plot, shown in Fig. 2.3, was generated from the tabulated values of NIST for dissociated and singly ionized nitrogen [91]. To measure a single wavelength from the spectral distribution of the APPT, a single line emission must be filtered out. The line must be completely isolated to ensure that the emission coefficient is not influenced by neighboring emission lines (either singly-ionized or doubly-ionized emission lines). From Fig. 2.3, the $528.12 \mathrm{~nm}$ N-I nitrogen emission line is a good choice based on spectral isolation. The closest neighboring emission lines are for singly ionized plasma and are sufficiently far away $(\sim 20 \mathrm{~nm})$ to be eliminated by optical filter.

To acquire the 2D chord-integrated intensity emitted by the APPT, a TSI, Inc. 29MP CCD camera was effectively converted into a 2D monochromator. The camera was affixed with an AF Nikkor 50mm lens with aperture set to f/22 and operated in 12 bit RAW mode and affixed with diopter lenses and an Andover Corporation 532 $\mathrm{nm}$ bandpass filter with a $\pm 10 \mathrm{~nm}$ wavelength range (FWHM). This filter transmitted 
the $528.12 \mathrm{~nm}$ emission line while reducing all other lines by a factor of $10^{4}$ or greater (OD4). This optical system was implemented to ensure that a maximum amount of the CCD camera's sensor gathered intensity data produced by the torch while still satisfying the parallel ray projection criterion, in order to achieve the spatiallyresolved motivation for 2DMIM. The camera was controlled by utilizing the imaging software Insight $4 \mathrm{G}$ and the exposure time was limited to $100 \mu s$. Both the aperture and exposure were chosen to prevent overexposure whilst achieving a high signal to noise ratio.

\subsubsection{Visual Methods for Hydrodynamic Experiments}

In addition to using spectroscopic measurements for plasma characteristics its also important to be able to qualitatively visualize the plasma. This is useful for capturing any hydrodynamic growth which may manifest itself during the RM or the MHD RM instabilities. A common optical diagnostic which allows experimentalists to capture both qualitative images and some useful flow characteristics is particle imaging velocimetry (PIV). PIV uses scattered light reflected from a particle field suspended in a gas. The particles in the gas phase are usually considered small enough to have negligible lag and do not disrupt the gas flow. Images are acquired at two times with a small $\delta t$ and statistical correlations are performed to determine the particle velocity field.

Typically this method requires particles to be injected either into the working gas, here plasma, or into the surrounding gas. For this work seeing the plasma phase presents a particular challenge. As mentioned previously the plasma consumed a thin tungesten wire, with a melting point greater than $3695 K$. This makes finding small particles which could survive the plasma environment difficult. There is also the 
matter of the electric interaction between any metal and the plasma which would have to be considered, making electrically conducting particles undesirable. As such, the non-ionized gas surrounding the plasma was seeded with non-conducting titaniumdioxide particles, particle diameters between $0.3 \mu \mathrm{m}$ and $1.0 \mu \mathrm{m}$, which were chosen based on their high melting and vaporization temperatures, $\sim 2,116 K$ and $\sim 3,245 K$ respectively. While this does not visualize the plasma itself, it does visualize the flow field surrounding the plasma giving potential statistics for edge vorticities and velocities which correspond well to the plasma flow characteristics.

The work presented in this chapter is simply to visualize the particle field and therefore the plasma (the non-illuminated region). In the future this technique will be further developed to acquire velocities at the plasma-gas interface. For now, the method images the plasma interface morphology in the $R-\theta$ plane within the shocktube facility for future shock-driven MHD-RM instability experiments. To develop this diagnostic technique, visualization of both the axial $(R-Z)$ and cross sectional $(R-\theta)$ planes were performed by imaging the particle-laden flow field surrounding the plasma while filtering out the plasma's emissions. The plasma's emissions were blocked by fitting the 29 MP CCD camera with a ThorLabs $532 \pm 1 \mathrm{~nm}$ laser line filter that transmitted only $532 \mathrm{~nm}$ laser light reflected from the particles. To illuminate the particles, a Nd:YAG laser, frequency doubled to $532 \mathrm{~nm}(\approx 200 \mathrm{~mJ} /$ pulse), was used with supplementary optics to generate a thin laser sheet to illuminate either the axial or cross-sectional plane. This technique had to be developed to be used in the Missouri Shock Tube Facility, as such an acryllic mock-tube was developed with a similar cross sectional area. Non-reflecting laser paper was used to eliminate reflections from the plastic and surrounding metal and the torch was mounted to the mock-tube for testing. This is shown in Fig. 2.4.

In the previous section, section 2.2.2, the CCD camera was made into a monochro- 
A)

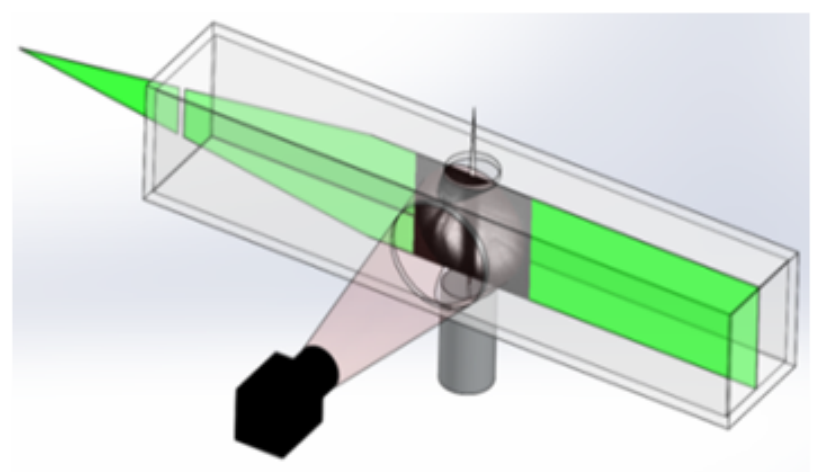

B)

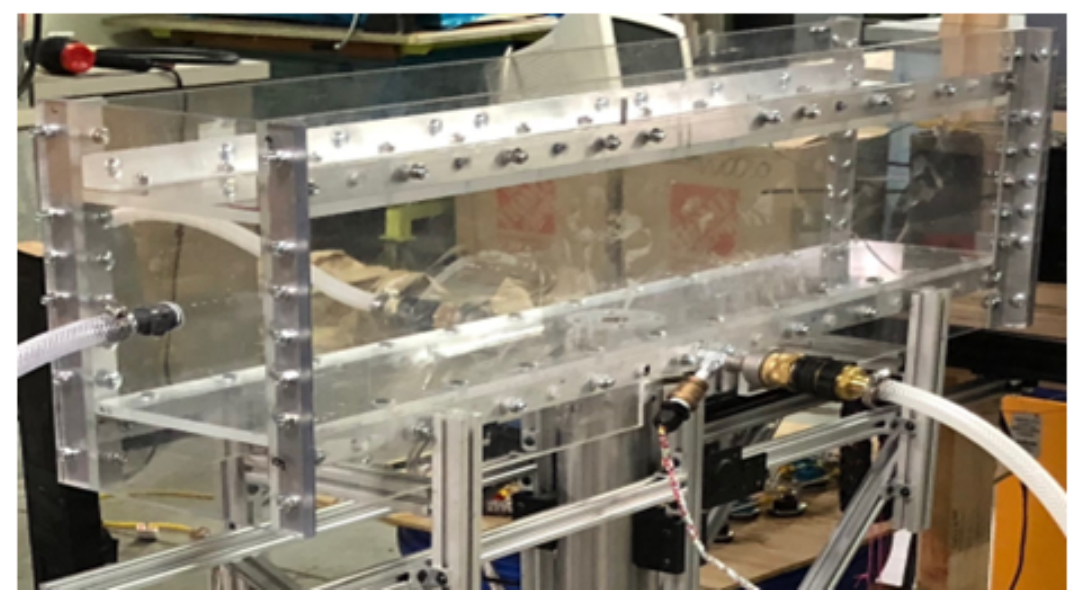

Figure 2.4: A) Conceptual representation of solid particle PIV test with R-Z Laser Plane illuminated B) Constructed Mock-Tube for PIV testing 
mator via the use of an Andover Corporation $532 \pm 10 \mathrm{~nm}$ wavelength bandpass filter. The purpose of this filter was to eliminate competing emission lines from the plasma source so that the 2DMIM could be used to find temperature and charge density. The purpose of this filter is to allow a high intensity signal from the plasma emissions to be seen by the camera. While this may seem beneficial for imaging the particle field as well, as the particles reflect the $532 \mathrm{~nm}$ Nd:YAG laser, the intensity of the plasma is many times greater than the intensity of the laser. This can be mitigated by using short wavelengths or small imaging exposure times, which will increase the intensity of the laser relative to the plasma. As such, another filter will be utilized to capture the scattered light from the particles and reduce the emissions from the plasma. A Thor Labs $532 \pm 1 \mathrm{~nm}$ wavelength bandpass filter will be used for imaging the interface. These two filters were tested using a variable wavelength light source to monitor what wavelengths were visable at what relative intensities, the results shown in Fig. 2.5. Here the solid lines represent the manufacturers curves while the shapes denote experimental data, this data was taken on broad wavelength spectrometers. The N-I emission line at $528.12 \mathrm{~nm}$ is also recordered. It can be seen that the Thor labs sees less than $1 \%$ emission of the N-I line, while experimental data indicates the Andover filter can see $\sim 20 \%$ of the relative intensity. Both allow over $50 \%$ relative intensity of $532 \mathrm{~nm}$. As such, for a better interface photo the Thor Labs filter will be used while the Andover filter will be used for 2DMIM. 


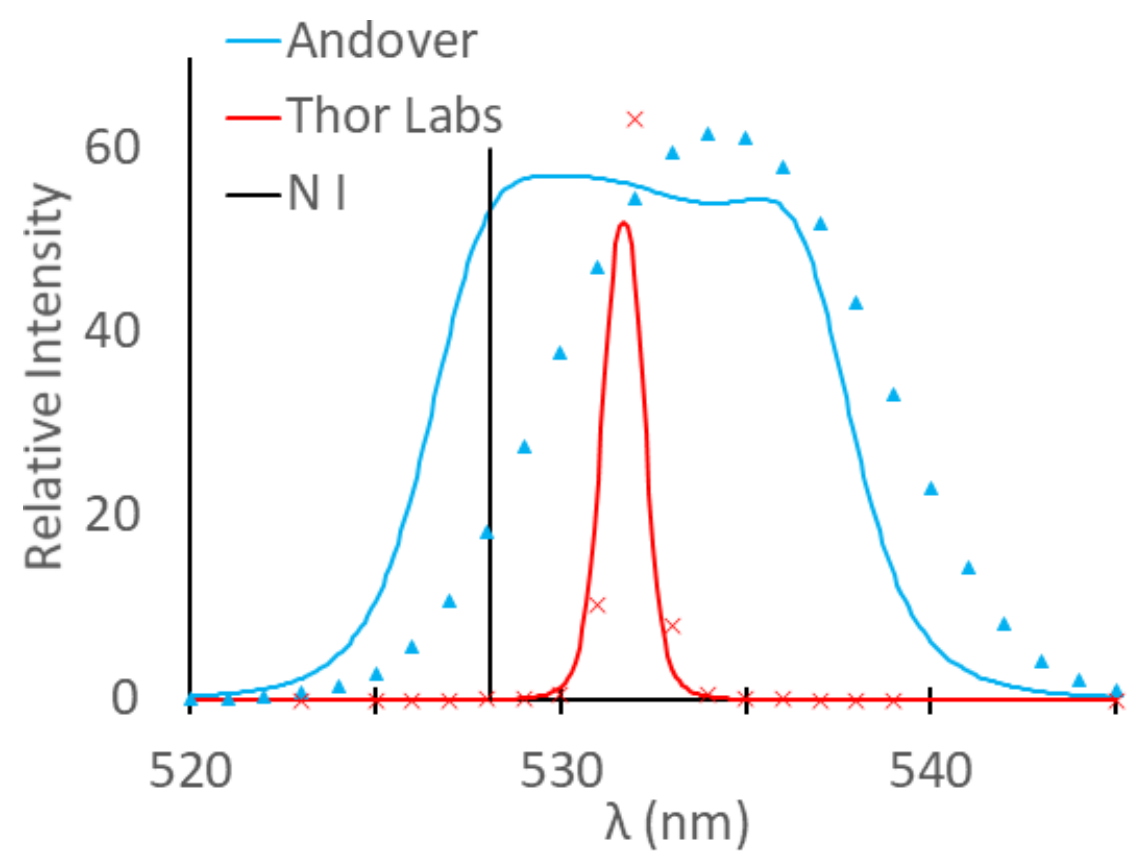

Figure 2.5: Comparison of the relative intensity at various wavelengths viewable through the (blue) Andover $532 \pm 10 \mathrm{~nm}$ wavelength filter and the (red) Thor Labs $532 \pm 1 \mathrm{~nm}$ wavelength filter. The solid lines indicate manufacturers specifications while the shapes represent experimentally measured data. The N-I $528.12 \mathrm{~nm}$ emission line, desirable for the 2DMIM, is also shown here 
Table 2.2: APPT Operating Parameters

\begin{tabular}{l|r} 
Arc Voltage: & $40-80 \mathrm{~V}$ \\
Arc Current: & $100-200 \mathrm{~A}$ \\
Axial Gas Flow Rate: & $1-10 \mathrm{SLM}$ \\
Tangential Gas Flow Rate: & $0.4-2.5 \mathrm{SLM}$ \\
& $10 \%-50 \%$ of total flow \\
\hline
\end{tabular}

\section{$2.3 \quad$ Results}

\subsubsection{Operating conditions of a nitrogen APPT}

There are three basic components utilized when developing and diagnosing an APPT, arc voltage, arc current, and gas flow rate (both axial and tangential flow rates). The parameters used are shown in 2.2. These three parameters were optimized to produce a stable laminar APPT with the longest jet length possible. The voltage and current in this table are limited by the $15 \mathrm{~kW} \mathrm{DC} \mathrm{power} \mathrm{supply.} \mathrm{That} \mathrm{is,} \mathrm{if} \mathrm{the} \mathrm{current} \mathrm{is} \mathrm{set}$ to $200 \mathrm{~A}$, the voltage can approach $80 \mathrm{~V}$.

Jet length was first measured using visible spectrum emissions, imaged by a DSLR camera. Fig. 2.6 shows a visible spectrum emission image of the torch in operation (right) and a calibration image for measuring the torch height (left). The image on the right was taken when the torch operated with an arc current of $170 \mathrm{~A}$, an arc voltage of $49 \mathrm{~V}$, and gas flow rates of 3.2 SLM axial and 0.6 SLM tangential. As shown in the right image, two distinct regions are visible. Region 1 depicts a luminous core surrounded by a luminous aureola, and thus represents the plasma region. The plasma region contains both ions and electrons, and is dominated by the various types of collisions, obeying the local quasineutrality assumption. Region 2 hosts both excited atoms and collisions but does not constitute a plasma, as there are no longer photons emitted at wavelengths corresponding to the dissociated or ionized 


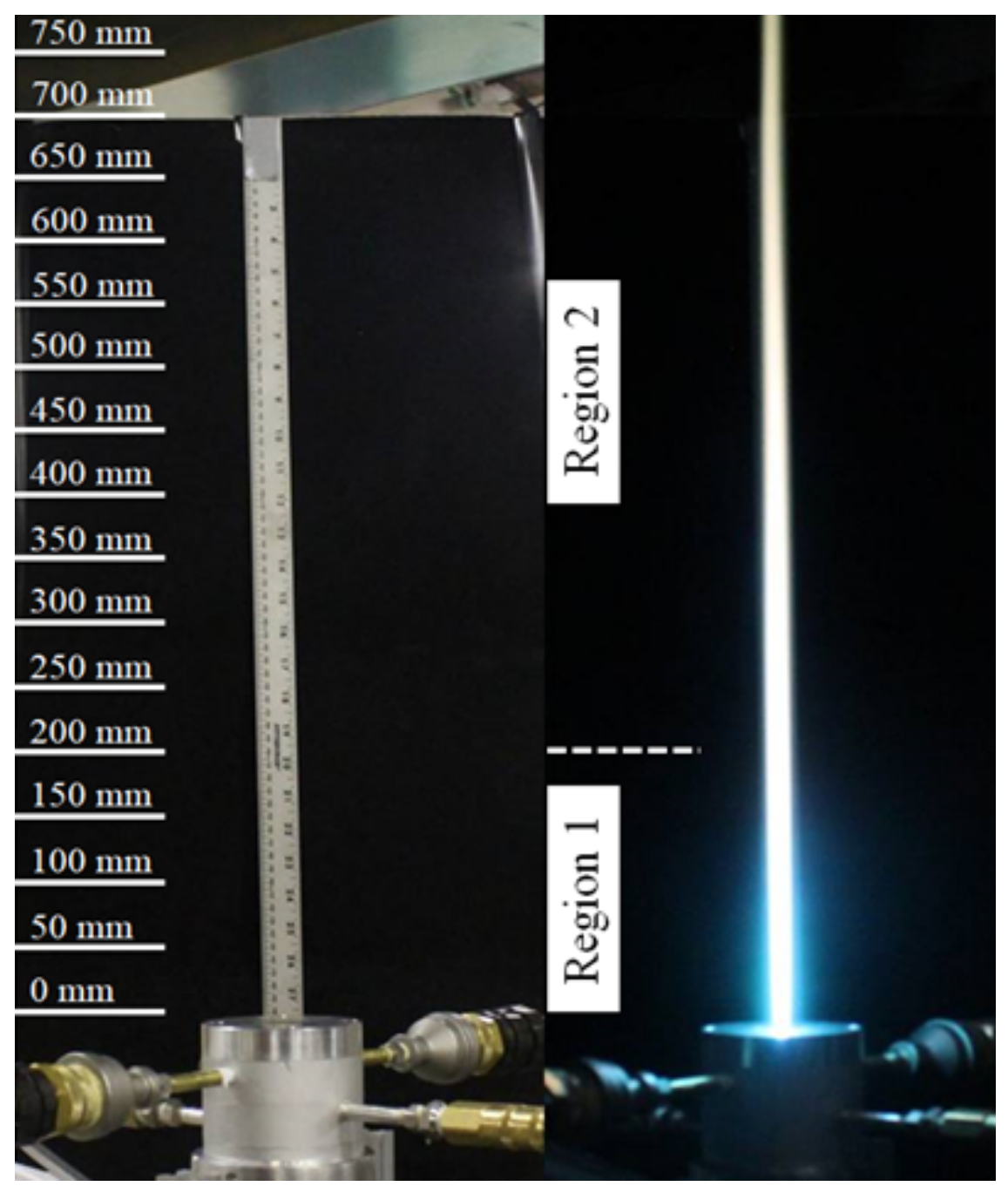

Figure 2.6: Calibration image (left) and APPT image (right) showing ionized region jet length (Region 1) at $20 \mathrm{~cm}$ and total jet length (Region 2) at $80 \mathrm{~cm}$ in length.

emission spectrum. This region 'glows' due to the hot incandescent gas. Ionized or dissociated molecules could exists in minute quantities beyond the bounds of region 2 but are undetectable by the current experimental setup.

Often distinguishing between what the plasma region and the incandenscent region is forgone because total jet length is denoted as plasma length. To separate these regions, images were taken with a DSLR camera for diagnosing the total jet length while simultaneously capturing images of singly ionized nitrogen emissions at 528.12 


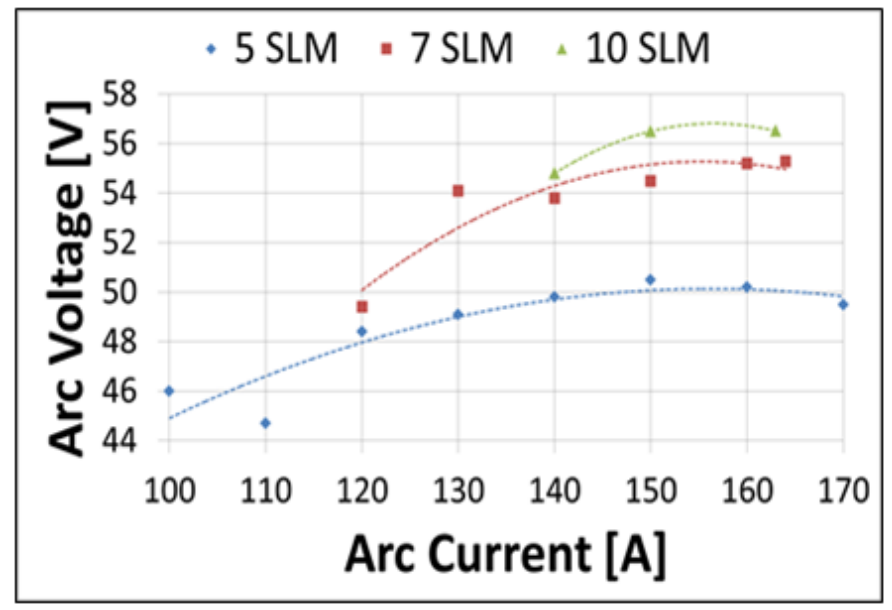

Figure 2.7: The voltage-current characteristics with respect to gas flow rate; as the current is increased the voltage increases as well

nm using a CCD camera and the 2DMIM to measure what is deemed the ionized region jet length. The ionized region jet length was determined by locating the highest point within the intensity field, captured by the CCD camera, that satisfied a minimum signal-to-noise ratio of $\mathrm{S} / \mathrm{N}=10$, that occurred consistently within a $10 \times 10$ pixel grid.

Shown in Fig. 2.7 are the V-I characteristics of the APPT for three gas flow rates. The three gas flow rates were 5, 7, and 10 SLM axial flow with the tangential flow rate kept constant at 1 SLM. The arc current was set by the HCDC power supply and the corresponding arc voltage was recorded. As seen in Fig. 2.7, increasing either the constant arc current or the gas flow rate increases the over all power into the flow.

Fig. 2.8 displays both the total jet length and the ionized region jet length as the axial gas flow rate was increased. The tangential gas flow rate and the power delivered to the arc were both held constant at 1 SLM and $15 \mathrm{~kW}$, respectively. The power was held constant by operating the Hgh Current Direct Current (HCDC) power supply in constant power mode. As the axial gas flow rate increased, the total jet length increased up to $3 \mathrm{SLM}$, followed by a diminishing return thereafter. This was 


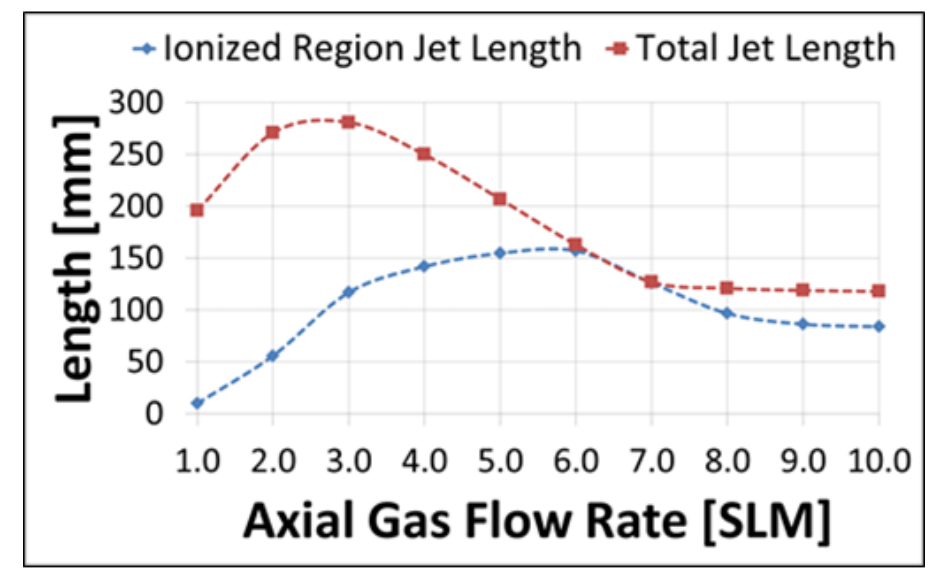

Figure 2.8: The total and ionized jet length with respect to increasing axial gas flow; as the gas flow increases the total and ionized region lengths converge. Similarly after a maximum axial flow rate of $\sim 6$ SLM both lengths begin to diminish due to turbulent effects

visually determined to be the result of a transition between laminar to transitional to turbulent flow regimes. Although the total jet length began to decrease as the axial flow rate increased past 3 SLM, the ionized region jet length increased until it became nearly equivalent to the total jet length at axial flow rates of 6-7 SLM. Axial flow rates of 7 SLM or greater resulted in turbulent effects dominating the flow causing an overall decrease in total jet length.

The last study performed when developing the APPT was to determine how the ratio of axial to tangential gas flow rates affected the total and ionized region jet lengths. This was accomplished by operating the torch HCDC power supply in maximum constant power mode and keeping the overall gas flow rate constant while varying the ratio of axial to tangential gas flow rates from $10 \%$ to $50 \%$. The resulting plot for using total gas flow rates of 3 SLM and 5 SLM is shown in Fig. 2.9. As this plot shows the jet length was not strongly affected by the flow ratio, though the longest jet lengths were achieved at a ratio of $70 \%$ axial to $30 \%$ tangential gas flow rate. 


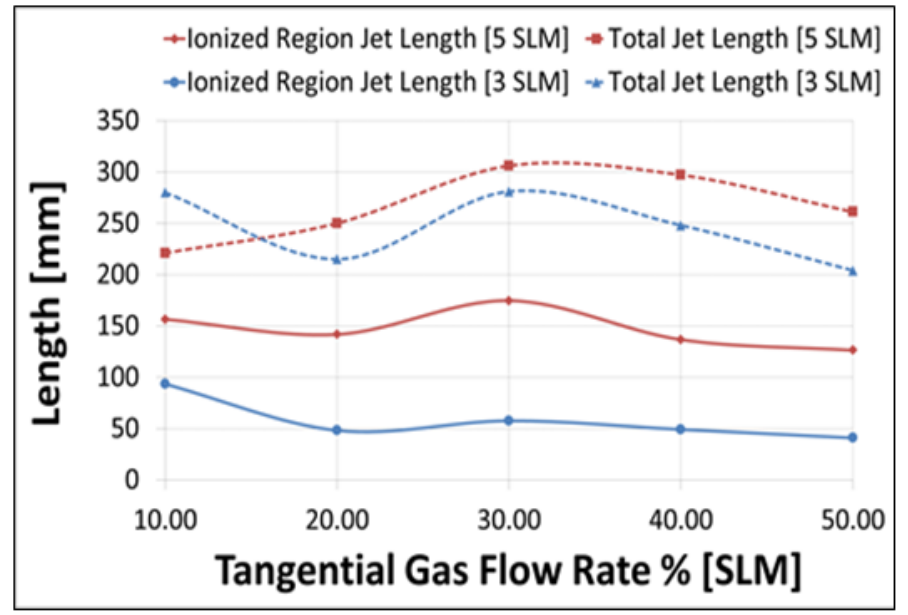

Figure 2.9: Ionized and Total Jet lengths with respect to tangential gas flow rates

While performing these studies other important factors, specifically repeatability of jet length observations, deserve consideration. The total jet length as shown in Fig. 2.6 does not coincide with the plots produced in Fig. 2.8 and Fig. 2.9. This is because the experiments in Fig. 2.6 utilized a newly constructed nozzle anode section and cathode tip, not yet subjected to ablation and erosion effects, which produces a longer more laminar jet. As the APPT is operated, the initial jet length decreases, but quickly ( $10 \mathrm{~min}$ ) settles into a stable condition where reproducible lengths are observable for many hours of operation. While the results portrayed in this diagnostic section are similar to results published in literature, the ability to implement fresh components for each jet length data point would be extremely arduous. Should new components be implemented, the results will scale accordingly; ultimately yielding the same overall trends for jet length optimization and ionized region vs. total jet length. Future design modifications may be made to maintain this initial longer total jet length. However, the ionized region, necessary for future MHD-RMI experiments, is not significantly affected by overall jet length. Thus, constantly implementing fresh components may not be beneficial. 


\subsubsection{Langmuir Probe Results}

It was mentioned previously that the Langmuir probe draws a current from the plasma due to the potential difference between the initial biased probe voltage, $V_{B}$ and the plasma while the data is read in by the DAQ. The measured data does not create the traditional langmuir curve, shown in Fig. 1.9. Instead, the initial data the voltage change with respect to time. Since the probe is sweeping, the initial signal gathered by the DAQ is the voltage bias to the probe as measured across resistors 1-5, Fig. 2.2. The signal changes as the sweeping probe enters into the plasma increasing as the probe moves from a less charged region into the center of the torch, and then decreases as the probe exits the center until it exits the plasma and returns to the bias voltage. An example of the data draw in this way is shown in Fig. 2.10 for a height of $25 \mathrm{~mm}$ in Z. Here two curves represent the signals drawn at different charges. The red line and left y-axis represent an experiment with a $V_{B}=10 \mathrm{~V}$. As the positively charged probe enters the plasma it draws negative charged species, decreasing the overall voltage signal. Similarly, the blue line and right y-axis represent a run with $V_{B}=-9 \mathrm{~V}$. As the negatively charged probe enters the plasma it draws a small amount of positive charge increasing its over all signal. The negatively charged probe draws only enough ions to increase its signal by less than $0.01 \mathrm{~V}$, meanwhile the positively charged probe draws enough electrons to drop its voltage a little over $0.5 \mathrm{~V}$. This may indicate that the floating potential is more negative than positive.

The probe was swept through the plasma, at some set height $\mathrm{Z}$, for a range of potentials a number of times and the curves shown in Fig. 2.10 were averaged together at each $V_{B}$. The local extreme of each curve was then taken to represent the maximum voltage at each point. The measured voltage, $V_{1}$, and $V_{B}$ were differenced and the circuit, Fig. 2.2, was used to calculate the necessary current drawn to produce the 


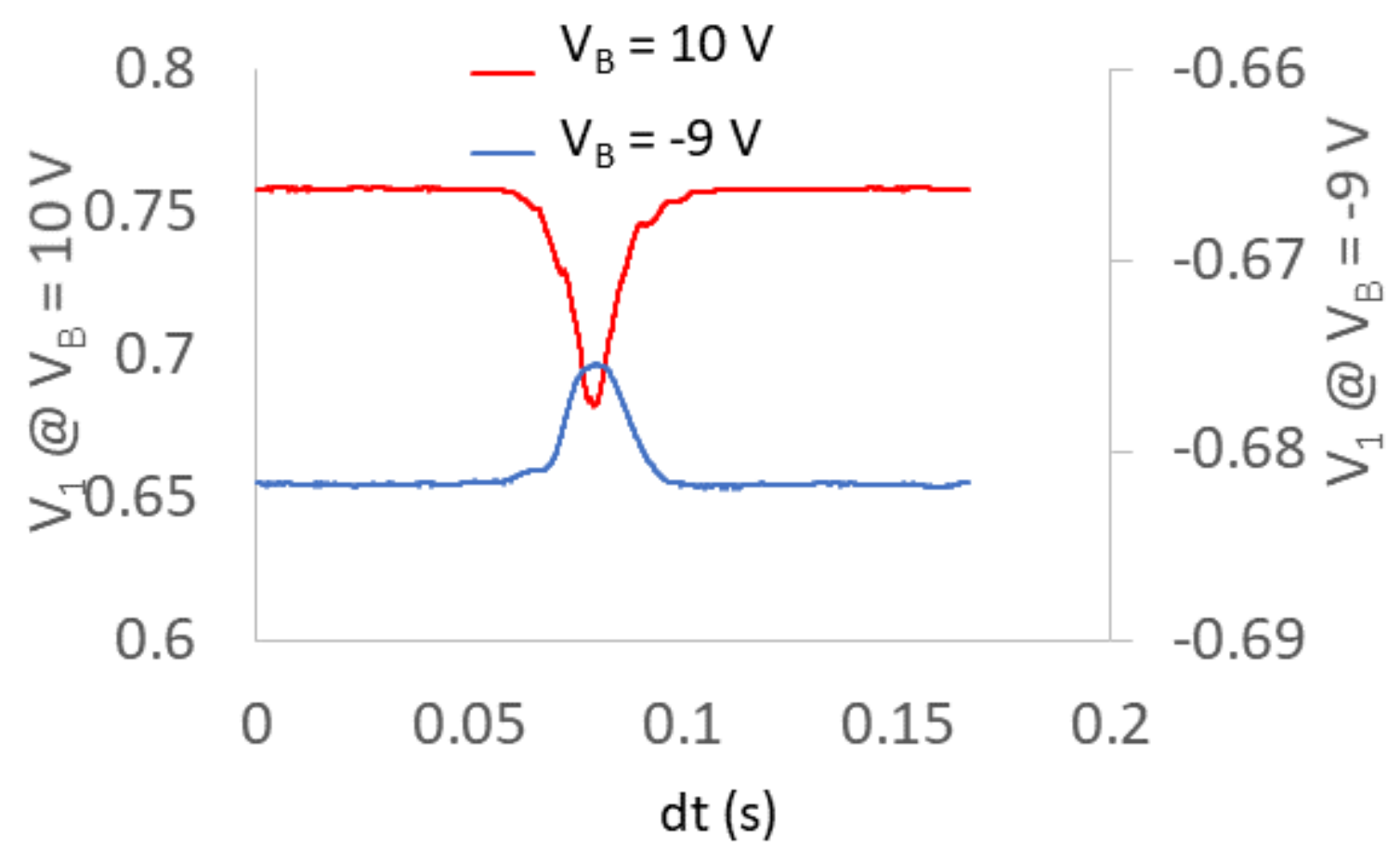

Figure 2.10: Langmuir probe data. These plots show the change in potential measured at the probe due to the current draw when $V_{B}=10 \mathrm{~V}$ (Red) and $V_{B}=-9 \mathrm{~V}$ (Blue). Here it can be seen that a positively biased probe draws negative current and decreases the overall potential while a negative probe draws positive current and increases the overall potential while the probe enters the plasma 
measured voltages. This extreme value was plotted against the initial $V_{B}$ in Fig. 2.11 A). This plot is supposed to be similar to the idealized Langmuir curve, Fig. 1.9. From this plot, one should be able to identify the ion and electron saturation currents as well as the plasma and floating potentials. However, the data gathered during these experiments make identifying these parameters difficult. From the plot it appears that neither of the saturation currents have been reached. The data is unsteady in both the ion saturation and electron saturation regions. For the electron saturation region, its been discussed that at high pressure applications the smaller mean free paths may cause the electron saturation current to be difficult to obtain. This is because as the probe draws negative charged species to its sheath, the positive ions begin to react. Normally, large mean free paths would mean that the positive particles couldn't set up their own space charge, but with the higher number density available to atmospheric plasmas the positve ions can draw additional electrons. Since the electrons have high mobility, they have the ability to affect the probe current as they're drawn to and through the positive sheath. As such, with increasing positive charge the langmuir probe may setup several layers of space charges, drawing an ever increasing electron current.

In addition to this complication, the ion saturation current has also not been found. Unlike the electron current, this parameter is easier to obtain in high pressure applications due to the ions low mobility. However, it was estimated from other published research that the max current drawn to the probe would be under \pm 1 A. As such, a simple DC power supply with a 1 A limit was purchased to bias the probe. From this data it can be seen that the current can exceed the equipments limitation, and it was indeed observed in experiments that when the current in the circuit rose above $1 \mathrm{~A}$, the power supply dropped its voltage and entered a constant power mode. This had the effect of disrupting the local charge around the probe and 
could greatly affect the data. As such, neither of the saturation currents were reliably found. Similarly the floating potential, or the $V_{B}$ which draws 0 current, is difficult to observe due to large number of voltages which gathered 0 current. Similary there is no apparent knee.

Ignoring for a moment that the data may not be entirely accurate, we can persue another avenue for analysis. Once the currents in Fig. 2.11 A) are found, the minimum current is subtracted from the data such that the curve is shifted up. The natural $\log$ of the currents is then taken and plotted against the biased voltage, Fig. 2.11 B). This plot removes the ion saturation current and instead should depict a linear region between where the voltage increases and draws some mixed current and the "knee", or the plasma potential. If this region is indeed linear, the inverse slope of this region can be used to determine the electron temperature in electron-volts. The conversion then can be made to kelvin. Figure 2.11 B) shows three different regions in an attempt to identify this temperature. The orange region is what would be the linear region, if the gray region were the "knee" or the plasma potential. The blue region indicates the unused section of the current. From this experiment, its clear that while current can be drawn and the bounds of the torch identified (Fig. 2.10) more work needs to be done to correctly calculate the V-I characterstics of the plasma with this method.

\subsubsection{D Monochromatic Imaging Method Results}

To fully utilize the 2DMIM, the computed total line emission coefficient must be plotted versus temperature, eq. 2.1. The normalized peak can then be used to find the normal temprature of the assumed charged species. These temperatures can also be correlated to the mol fraction of neutral, dissociated, and charged components of 

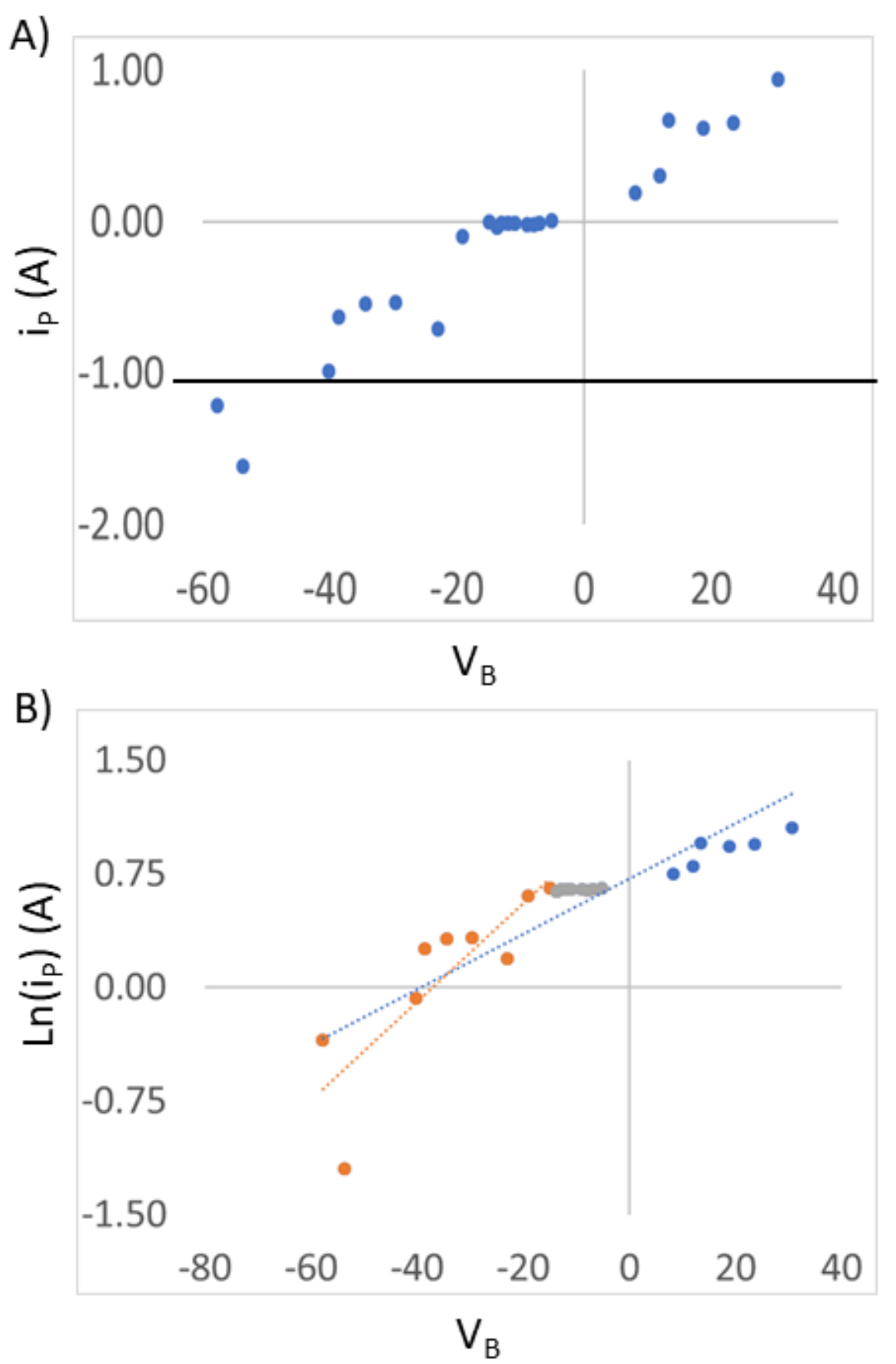

Figure 2.11: Top: Raw Langmuir Data, Bottom: The log natural of translated Langmuir data. The data has been shifted up by the estimated ion saturation current in an effort to find the electron temperature. Here the data can be seen to be scattered and difficult to correlate with regards to the idealized langmuir data previously shown 
the plasma species, allowing one to estimate what percent ionization exists at the temperatures. Its also necessary to get a calibration image from a transferred torch. These steps were not covered in the previous discussion and thus wont be presented here. However, they are important to the method and additional information can be found in the paper this section is based off of [2].

Once the optical system is calibrated, spatially resolved 2-D temperature and degree of ionization profiles could be made for the APPT in the non-transferred arc configuration. Performing the Abel inversion[2] on the intensity image was done per row of data such that the maximum intensity value was taken to correspond to the centerline axis of the torch. The left and right side of the image (relative to the centerline of the torch) were then Abel inverted separately so as to preserve some of the torch asymmetry. The left image in Fig. 2.12 shows the bandpass filtered image (528.12 nm line emission), temperature (center) and degree of ionization (right) profiles for the APPT operating at 2.1 SLM axial and 0.9 SLM tangential gas flow rates with an arc current of $200 \mathrm{~A}$. These parameters were chosen to ensure that the entirety of the plasma containing region would be captured and to ensure a high level of spatial resolution ( $540 \times 2100$ pixels) would be achieved. Ionized region jet lengths greater than the one imaged in Fig. 2.12 would ultimately result in the entirety of the plasma containing region not being captured by the calibrated optical system. Larger torches were imaged but the entirety of the ionized region was not visible.

The total jet length achieved for these conditions was $280 \mathrm{~mm}$. However, the ionized region jet length was only $64 \mathrm{~mm}$ for this specific case, as shown in Fig. 2.12. As the plasma is ejected out of the nozzle, the intensity values measured decreases both axially and radially. This is due to recombination, de-excitation, and diffusion effects that return the ionized gas $(\mathrm{N}++\mathrm{e}-)$ to its dissociated (atomic nitrogen, $\mathrm{N}$ ) and then initial (molecular nitrogen, N2) state. The maximum plasma temperature occurred 


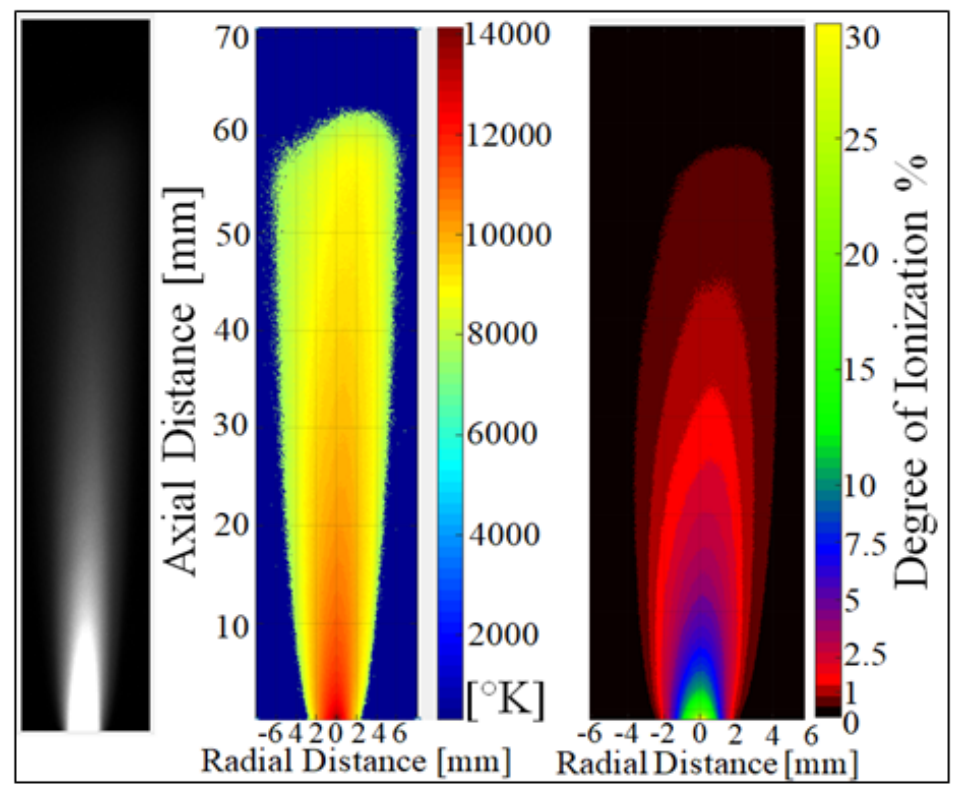

Figure 2.12: The 2DMIM results showing the monochromatic raw image (Left), the $R-Z$ temperature distribution (Middle), and the degree of ionization as a function of temperature (Right) for axial and tangential flow rates of 2.1 and 0.9 SLM respectively and an arc current of $200 \mathrm{~A}$

at the exit of the nozzle and was found to be nearly that of the normal temperature, $14,000 \mathrm{~K}$ with a maximum degree of ionization of $\sim 27 \%$. This is to be expected as arc root attachment within the nozzle is very close to the exit. Additionally, the radial plasma temperature profile just above the nozzle exit is shown in Fig. 2.13. Previous monochromatic and spectroscopic investigations of APPTs have depicted a Gaussian profile when examining the radial temperature distribution. Implementing the 2DMIM however allows for more accurately defined profile to be achieved that shows a non-Gaussian profile. At the edge of the plasma-atmosphere interface there is a steep defined gradient where the temperature drops sharply from $\sim 7450 \mathrm{~K}$ to equilibrium with atmospheric (room) temperature $\sim 300 \mathrm{~K}$. This is because as the temperature drops radially the charged and dissociated nitrogen no longer has the energy necessary to exist in an unstable state. Thus, as it cools it recombines to form 


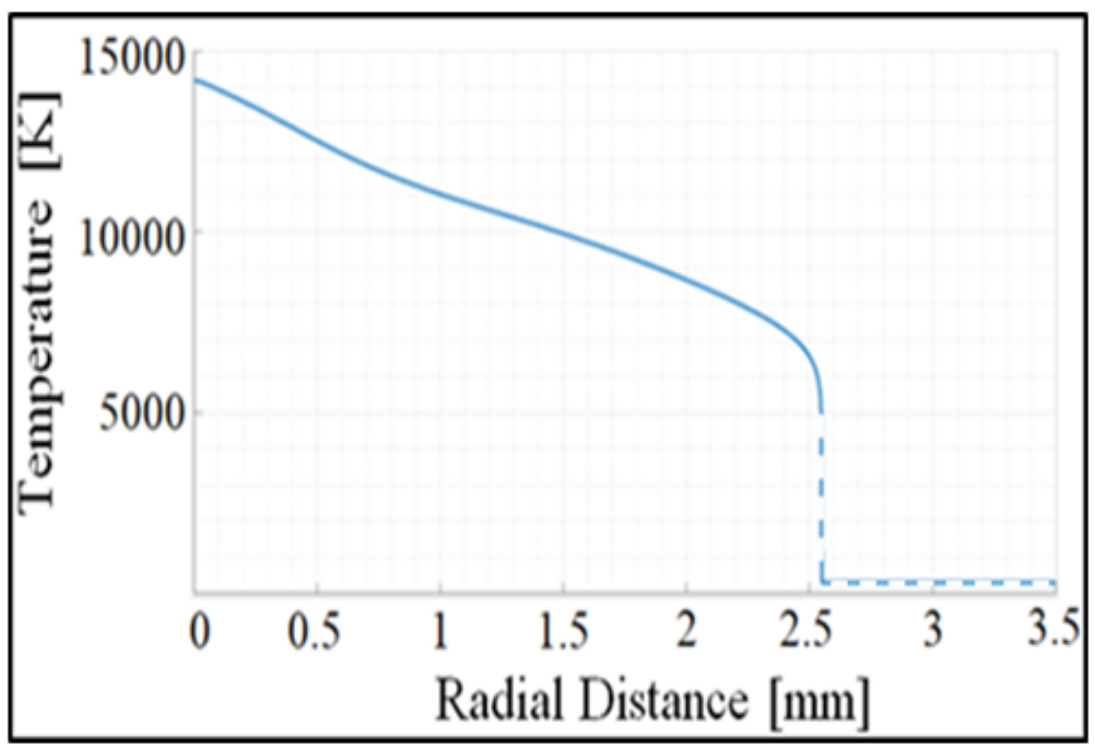

Figure 2.13: Radial Temperature profile at nozzle exit for APPT experiment shown in Fig. 2.12. Dashed line indicates plasma temperature measurements with unresolvable N-I concentrations

stable molecular nitrogen and there is no longer any significant presence of emitting electrons or ions.

\subsubsection{Plasma Imaging Results}

The plasma emission was found to be much greater than the illuminated particle field over a longer exposure $(100+\mu \mathrm{s})$ as seen in Fig. $2.14(\mathrm{a})$, necessitating the use of a narrow bandpass filter. Keeping the CCD camera exposure (duration of intensity data acquisition) to $100 \mu \mathrm{s}$ (near the limit of the camera) also minimized the continuous plasma emission intensity integrated over the duration of the exposure. Since the duration of the laser pulse, and thus particle field illumination time, is approximately $4 \mathrm{~ns}$, the reflected laser signal compared to the plasma emissions will increase as the exposure time is decreased to 4 ns. From Fig. 2.14 (b), it can be seen that plasma emission is still visible while using the $532 \pm 10$ filter but that there is a large reduction 


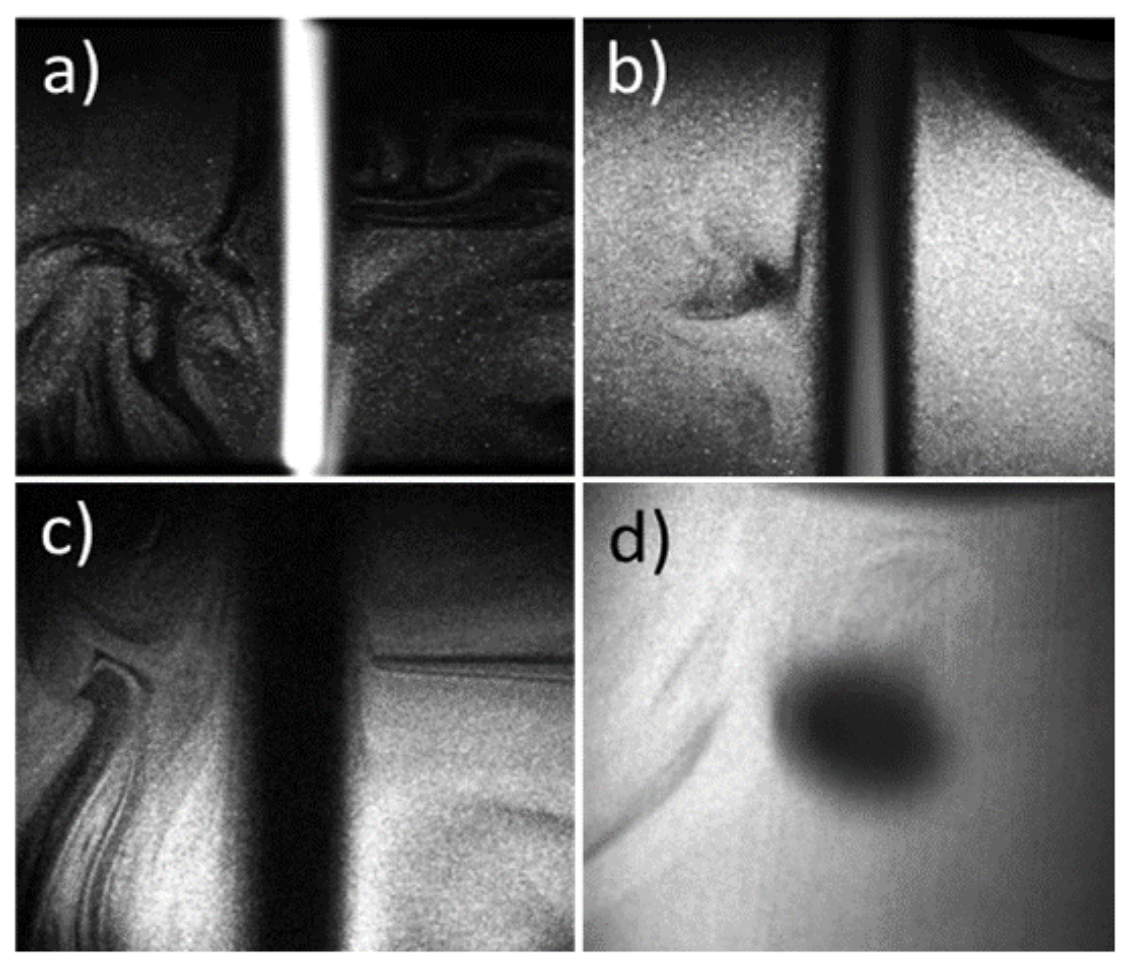

Figure 2.14: Plasma-Particle Interface Imaging results: a) $R-Z$ plane imaged without any filter b) $R-Z$ plane imaged with $532 \pm 10 \mathrm{~nm}$ Andover bandpass filter c) $R-Z$ plane imaged with $532 \pm 1 \mathrm{~nm}$ Thor Labs bandpass filter d) $R-\theta$ plane imaged with 532 Thor Labs bandpass filter. Both c) and d) give the best results for eliminating the emissions from the plasma for future hydrodynamic experiments

in the illumination intensity of the plasma compared to using no filter, Fig. 2.14 (a). Here, the laser illuminated particle field intensity is on the same order to that of the plasma emission intensity.

Additionally, there is a boundary layer evident between the plasma and the surrounding particle-laden atmosphere. This is consistent with the results shown in Fig. 2.13 where the particles that would otherwise be present, have vaporized due to the high temperature imposed on them by the plasma. The particles melting temperature is $2,116 \mathrm{~K}$ while the vaporization temperature $3,245 \mathrm{~K}$. Using simple $1 \mathrm{D}$ calculation for particle heating and phase change the particle life is estimated to be on the order 
of $100 \mu \mathrm{s}[92,93]$.

The elimination of the plasma emission is visible in Fig. 2.14 (c), where the dark column in the middle of the figure is the plasma flow region. Additionally, the illuminated particles appear only in a circular section of the camera field-of-view with the narrow bandpass filter affixed even though particles in the full field-of-view are illuminated by the laser sheet. This is attributed to the angle of incidence of scattered (reflected) light from the particles (rays), as the wavelengths passed by the narrow bandpass filter shift towards the blue spectrum (the center wavelength of $532 \mathrm{~nm}$ is blocked) as the angle of incident light decreases. Thus, less particle illumination (i.e. laser light reflected off the particles as convergent or divergent rays) is passed through the laser line filter in regions away from the center of the field-of-view. In Fig. 2.14 (ac), there are also structures formed outside the plasma boundary (shown in dashed red lines) which occur due to particle injection into the atmospheric surroundings within the MT section.

To visualize the $R-\theta$ plane, the laser sheet was rotated $90^{\circ}$, the camera repositioned to capture the new laser plane, with the result shown in Fig. 2.14 (d). As with many PIV experiments implemented to investigate hydrodynamic instabilities such as the RM instability, a cross sectional image of the $R-\theta$ plane is desired to capture the instability as it evolves in time. Since the $R-\theta$ plane is not able to be captured with the camera positioned directly above the plasma jet column, the image was acquired at an off-axis angle from the plasma column (perpendicular to the laser sheet) and the image was corrected using for angle. Therefore, developing this method can be used for PIV measurements in future MHD-RM instability experiments once implemented in the FMSTL shock tube. 


\subsection{Conclusions}

The development and diagnosis of a non-transferred DC arc atmospheric pressure plasma torch was presented in this chapter. A long laminar torch was developed and gas flow rates, arc currents, and arc voltages were explored. It was found that the jet length and total power are functions of axial flow rate. It was also found that the total jet length can be divided into two regions, a plasma region and an incandescent region. As the axial flow rate is increased the plasma region grows as the total jet length decreases until the torch transitions to turbulence and both decrease in size. Tangential flow was also explored and found to have a minimal effect.

Diagnosis of the plasma was performed utilizing a single langmuir probe, 2D Monochromatic Imaging Method, and a particle Mie scattering method (particle image velocimetry). While the Langmuir Probe measurements were unsuccessful, the experiment as a whole seemed promising. It is recommended that the equipment be upgraded so that the plasma parameters can be found. It may also be beneficial to persue a double langmuir probe configuration, so that the biased and measure voltages are based on the circuit and may provide more stable readings. Despite the langmuir probes failure, the 2DMIM was able to provide electron temperatures and charge density. This data can be used for future experiments and simulations. Similarly, the PIV-like Me scattering images can be used to capture the interface in $R-Z$ and $R-\theta$ and are ready for use. Once MHD-RM instability experiments are run this method can be converted to full PIV and provide data to validate simulations. 


\section{Chapter 3}

\section{D Simulations of the Magnetohydrodynamic Effects in a Shock-Accelerated Gas Cylinder}

This chapter presents 2D simulations on a cylindrical Richtmyer-Meshkov Instability in Magnetohydrodynamics. It was adapted from a publication in Physical Review Fluids, [3]. Three studies are presented in an effort to quantify and qualify the evolution of the MHD RM instability by varying the magnetic field orientation, strength of the magnetic field, and strength of the shock wave driving the instability. The orientations considered herein are either parallel or perpendicular to the shock wave motion. The second study varies the magnetic fields between 100, 250, and 500 gauss $(\mathrm{G})$; while the third study considers incident shock wave Mach numbers $M=1.2, M=1.66$, and $M=2.2$. These parameter ranges were selected to be easily achievable in experiments while the interface perturbation was selected such that its evolution is independent of either the shock wave or magnetic field orientations independently. It was found that the MHD RM instability evolution is dependent upon the magnetic field orientation relative to the shock transit direction as well as their 
individual magnitudes. This is because the mechanism of suppression, attributed to Alfven waves, is a function of the magnetic field strength and the orientation of the magnetic field while the mechanism of RM instability evolution, baroclinic vorticity deposition, is a function of the Mach number. Stronger magnetic fields were found to provide greater mixing suppression and have significant effects on RM instability-like interface morphology. Finally, increasing the shock wave strength generated competing effects between higher RM instability vorticity deposition and greater vorticity removal from the interface by faster Alfven waves.

In consideration of the research done in literature previously and with future hydrodynamic experiments in mind, this section will explore a cylindrical interface of perfectly conducting gases in the presence of a magnetic field through simulations. The simulations presented are $2 \mathrm{D}$ in cartesian coordinates and consider a $2 \mathrm{D}(\mathrm{R}$ $\Theta)$ slice of a cylinder interface with a planar shock wave. The nomenclature of cylindrical will be adopted with consideration for previous RM instability literature on both experiments and simulations. The simulation conditions were selected to be achievable in experiments at the University of Missouri Fluid Mixing Shock Tube Laboratory (FMSTL) [84]; additionally the cylindrical interface was chosen for its unique geometry. The nature of the cylinder is such that it provides a double interface $[18,19,20]$ and does not have a preferential direction with respect to the shock wave or the magnetic field. However, the growth of the cylindrical interface exhibits a dependence on the orientation of the magnetic field relative to the shock wave. In Fig. 1.7, the shock wave is shown traveling in the $Y$ direction. This means the site of maximum vorticity deposition is $|\alpha|=\pi / 2$. However, if the shock wave direction was changed such that it was traveling in $X$ the maximum vorticity would occur on $|\alpha|=0$, or orthogonal to the shock wave direction. Assuming the interface is allowed to evolve uninterrupted by boundaries and magnetic fields the evolution of 
the cylindrical RM instability would be the same between these two cases.

\subsection{Computational Enviroment: FLAG}

The 2D simulations presented in this work were performed using the hydrocode FLAG [94, 95]. FLAG is a multi-material multi-physics hydrodynamics code developed at Los Alamos National Laboratory (LANL). FLAG includes a fully unstructured grid mesh, allowing an arbitrary polyhedral mesh, and can function as an Arbitrary Lagrangian-Eulerian (ALE) code utilizing a Lagrangian hydrodynamics step followed by an optional relaxation and remapping step [96, 97]. The relaxation algorithm in the current work is a Laplacian-type smoothing, and is performed every cycle. The FLAG remapping step uses the Flux Corrected Transport (FCT) algorithm of Boris and Book $[96,98]$ to achieve second-order accuracy for smooth solutions, while preserving monotonicity at discontinuities and ensuring conservation. The implementation is directionally unsplit in 2D/3D, based on Zalesak [99] and limited gradients are computed with the unstructured Barth-Jespersen limiter [100]. Experience suggests both FCT and gradient-limiting are necessary for high-strain-rate flows on highly deformed meshes. Multi-material interfaces are reconstructed using Youngs' volume of fluid method (VOF) [101].

FLAG utilizes the ideal MHD equations, shown in the Introduction as Eqs. 1.10 through 1.15 and utilizes a poisson solver for divergence control. In addition to treating the materials as perfect conductors, FLAG also treats all fluids as ideal gases, gamma law equation of state shown as Eq. 1.9. While FLAG has resistivity capabilities, this work does not consider two fluid MHD, resistivity, or Hall effects. 


\subsubsection{Simulation Parameters and Initial Conditions}

The simulation parameters for all cases are given in Table 3.1. All simulations consider a cylindrical interface with a diameter of $2 \mathrm{~cm}$. This interface is perfectly conducting nitrogen gas at $2500 \mathrm{~K}$. The interface bulk temperature was selected in regards to the process of ionizing nitrogen at atmospheric conditions. The interface is surrounded by air at $300 \mathrm{~K}$, the unshocked region is initialized at standard atmospheric pressure. Figure 3.1, an annotated pseudo-color of plasma mass fraction (species 2 per the Atwood number definition) given as $\Upsilon_{P}$, shows the initial conditions common to all simulations. Here the shock wave is represented by a planar black line and with an arrow showing the direction of motion. A diffuse interface was chosen to due to the large temperature difference between the interface and its surroundings. This figure also has a thin line visible in the diffusion layer which represents the 5\% plasma species contour line and is visible in all images for clarity. While this figure shows the interface, it does not show the boundaries in either the $X$ or $Y$ directions. This slice shows the interface at time $t=0$ and is a $5 \mathrm{~cm}$ by $5 \mathrm{~cm}$ area centered around the interface, this scheme will be used repeatedly throughout this work. The full domain is $20 \mathrm{~cm}$ by $2000 \mathrm{~cm}$ with reflecting boundaries in $X$ and $Y$ respectively. The resolution in all simulations is 100 nodes per centimeter (200 nodes per cylinder diameter) and all images are visualized using the computational post-processing software ENSIGHT, developed by Computational Engineering International, Inc.

To investigate the effects of the magnetic field on the MHD RM instability, simulations were conducted over a wide range of parameters. The parameter ranges were selected with the design of future experiments in mind. The first parameter considered is the magnetic field orientation where the traditional RM instability, $B=0$ is compared to cases with a perpendicular, $B=B_{\perp}$, or parallel magnetic field, $B=B_{\|}$, 
Table 3.1: Initial Conditions for 2D Simulations

\begin{tabular}{|c|c|c|c|}
\hline 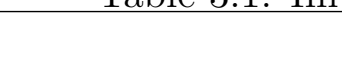 & $B$ & $B_{\|}$ & $B_{\perp}$ \\
\hline$B(\mathrm{G})$ & 0 & $100,250,500$ & $100,250,500$ \\
\hline$\beta$ & - & $2550,410,100$ & $2550,410,100$ \\
\hline$P_{0}(\mathrm{~Pa})$ & & 101,325 & \\
\hline$T_{1}(\mathrm{~K})$ & & 300 & \\
\hline$T_{2}(\mathrm{~K})$ & & 2500 & \\
\hline$\rho_{\text {air }}\left(\mathrm{kgm}^{-3}\right)$ & & 1.12 & \\
\hline$\rho_{N_{2}}\left(k g m^{-3}\right)$ & & 0.136 & \\
\hline$A$ & 0.79 & & \\
\hline$M$ & 1.2 & 1.66 & 2.2 \\
\hline$U_{p s}\left(\mathrm{~ms}^{-1}\right)+/-1 \%$ & 108 & 311 & 514 \\
\hline$D_{i}(\mathrm{~cm})$ & & 2 & \\
\hline
\end{tabular}

of 500 Gauss $(\mathrm{G})$. These cases all share $M=1.66$, a Mach number realizable within our shock tube [84], and $A=0.79$. This study is presented in Section 3.2.1. After the orientation effects are presented, the magnetic field strengths for both orientations are varied as 100, 250, and $500 \mathrm{G}$. This range of field strengths was selected for two reasons; first, this range is easily achievable for experimentalists even when considering a shock tube domain, and second, if observable suppression occurs at weak magnetic fields in ideal MHD, it's possible that even with resistivity effects a strong magnetic field is unnecessary to suppress the instability. The results for this study are shown in Section 3.2.2. Similar to Section 3.2.1 this section has $M=1.66$ and $A=0.79$.

The final parameter investigated within this work is the Mach number. The two previous studies explored the effects of the magnetic field, but it is also of interest to see how the hydrodynamics variables may affect the evolution of this interface in MHD. Sections 3.2.1 and 3.2.2 utilize $M=1.66$, and so this study will expand the Mach numbers to include $M=1.2$ and $M=2.2$. These numbers were selected as they are within the range of our shock tube, and the post-shock velocity increases by approximately $200 \mathrm{~ms}^{-1}$ with increasing $M$. This study will only consider the $B=0 \mathrm{G}$ 


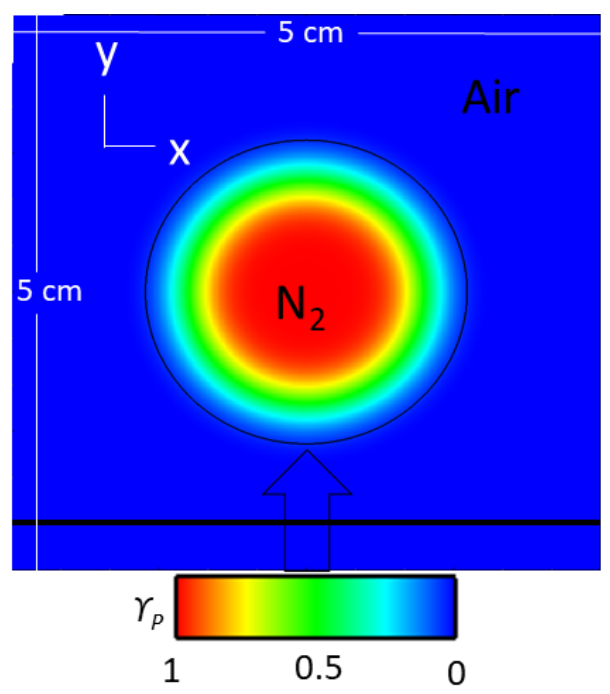

Figure 3.1: 2D species pseudo-color representing all initial conditions for this work. Here the shock wave is traveling in the $+Y$-direction and the interface considered as a $2 \mathrm{~cm}$ diameter cylinder of $N_{2}$ at $T=2500 \mathrm{~K}$. The magnetic field, if present may be parallel (along $\mathrm{Y}$ ) or perpendicular (along $\mathrm{X}$ ) to the direction of propagation of the shock and is not shown here. The image is centered about $y=0$.

and $B=B_{\perp}=500 \mathrm{G}$ cases. As this work seeks to study a shock driven hydrodynamic instability in magnetohydrodynamics, it should be noted that the inclusion of MHD effects can alter the shock jump equations due to the addition of the magnetic pressure term [102]. However, the initially single directional magnetic fields presented here do not meaningfully change the post shock conditions. This was observed by looking at the post shock scalar fields for temperature and pressure, which vary up to $3 \%$ from the expected conditions, and the post shock velocities, which vary up to $2 \%$. Therefore, the authors feel confident in comparing $500 \mathrm{G}$ magnetic fields cases to the traditional RM instability at different Mach numbers in this work.

When discussing the results, a naming convention becomes necessary in the face of such a wide parameter space. As such, the following naming convention will be used. Each case will be denoted in the following format $M \# B_{\text {dir }} \#$ where $M \#$ indicates the Mach number and the \# is replaced by a 1.2, 1.7, or 2.2 for a Mach number of 1.2 , 
1.66, or 2.2 respectively. The second half of the nomenclature represents the magnetic field. Here, dir can be either $\perp$ or $\|$ to represent a field perpendicular or parallel to the shock wave direction. The \# will be either 10, 25, or 50 for a field of 100, 250, or 500 G. Lastly, there are cases where the magnetic field will be set to 0 to recover the traditional RM instability and will be denoted by $B 0$. For example, $M 1.7 B 0$ stands for a case which considers a Mach number of 1.66 and no magnetic field.

\subsection{Results}

For a traditional cylindrical RM instability with these initial conditions, $M 1.7 B 0$, significant RM instability developement occurs as early as $t=0.5 \mathrm{~ms}$ post shock. However, it is desirable for each case to be considered using a standard temporal scale, especially across the various Mach numbers. As such, a non-dimensional time common in RM instability literature will be used for all cases. This time, given in Eq. 3.1 , is a function of time, the interface amplitude, and the shock wave transit speed or $t, \eta_{0}$, and $w_{i}$ respectively. Since the interface is a cylinder the interface amplitude is replaced by its diameter, thus $\eta_{0}=d$. Therefore, all simulation results will be presented using $\tau$ for consistency.

$$
\tau=\frac{t w_{i}}{d}
$$

\subsubsection{Magnetic Field Orientation}

The first study presented in this work will compare the $M 1.7 B_{\perp} 50$ and $M 1.7 B_{\|} 50$ cases to the traditional RM instability case denoted as $M 1.7 B 0$. Figure 3.2 contains the species pseudo-color with respect to $\tau$ and has been annotated in order to aid 
discussion. At early time $M 1.7 B 0$ shows two large vortices and a thin bubble front. As the simulation progresses, this bubble front dissipates and the vortices begin mixing. By late time, the tight vortex structures have experienced significant mixing leaving almost no pure plasma species. The most similar MHD RM instability case is $M 1.7 B_{\|} 50$, where the magnetic field is parallel to the shock direction. This case shows similar evolution at early time but with less developed vortices and a thicker bubble front. At mid-time, large vortex structures have formed but exhibit less mixing and a larger area where the plasma species is preserved when compared to $M 1.7 B 0$. There is also a morphological difference in the vortices, as tear drop like spikes form near the leading edge of the interface. At late time, the vortices and spike-like structures have been stretched as the interface grows. However, there is a significant reduction in species fraction dissipation, which suggests a reduction in mixing with the surrounding gas.

The middle column shows results from $M 1.7 B_{\perp} 50$. Immediately a difference can be seen between this case and the other two as early as $\tau \approx 3$. Here, a thick bubble front is present with almost no vortex development. By mid-time the morphology is significantly different than the other two cases. No vortex features are present, instead there is a thick bubble front structure. There is also significantly less mixing, with a strong concentration of pure plasma. At this time, the bubble front also exhibits a convex leading edge and a concave trailing edge. By late time the configuration of these regions have reversed. That is, the interface has inverted itself. Similar to mid-time, there is almost no change in the species pseudo-color to suggest additional mixing. Therefore, both magnetic field orientations show a decrease in qualitative mixing and RM instability evolution when compared to the traditional RM instability, with $M 1.7 B_{\|} 50$ showing similar morphology with reduced qualitative mixing and $M 1.7 B_{\perp} 50$ showing no discernible evidence of the RM instability. 


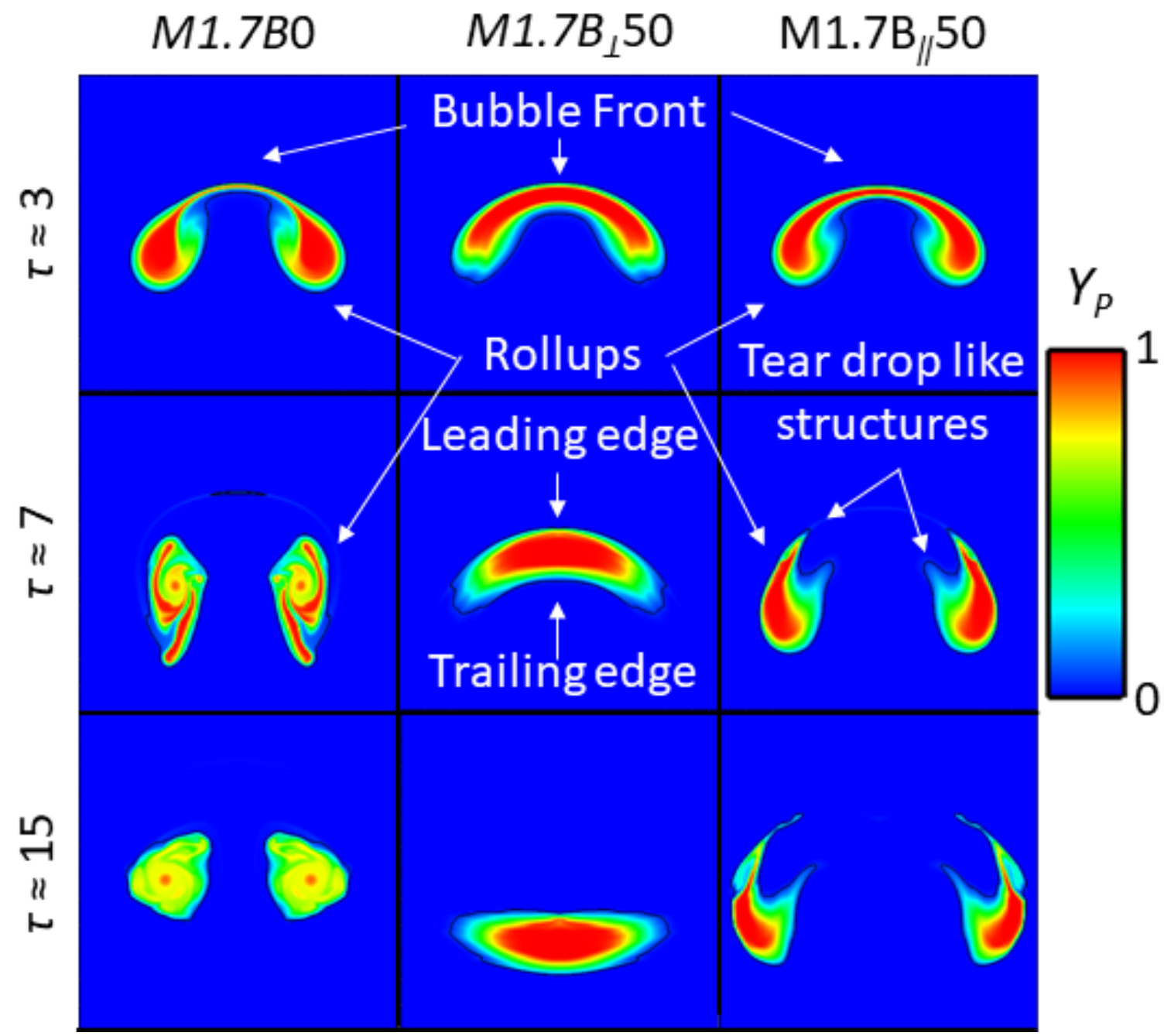

Figure 3.2: Magnetic orientation study; pseudo-color of species for the $M 1.7 B 0$, $M 1.7 B_{\perp} 50$, and $M 1.7 B_{\|} 50$ cases with respect to $\tau$. The images are centered about $y$, respective to $\tau$ at $y(\tau \approx 3)=3.11 \mathrm{~cm}, y(\tau \approx 7)=7.64 \mathrm{~cm}$, and $y(\tau \approx 15)=15.55$ $\mathrm{cm}$. 
It's common when considering the RM instability to try to quantify the mixing. However, this can be a challenge as one must determine the amount of mixed fluid versus the amount of entrainment in the system. The mixedness parameter, given in Eq. (3.2) is often used in RTI literature [103], and is considered as the ratio of the mixing half-width layer, $h$, to the half-width homogeneous mixing layer, $h_{\text {hom }}$, or simply the ratio of mixing to entrainment. These widths are functions of the mass fraction of the plasma species, $\Upsilon_{P}$, where an overbar and ' denote an average and a fluctuating component respectively. The mixedness is bounded between 0 , for a case in which no mixing has occurred, and 1 where two species have mixed completely. To calculate $\Xi$ the averages were taken in $Y$ while the integrals were taken in $X$. This parameter is shown in Fig. 3.3. Since the qualitative evolution is indicative of mixing, it is expected that the more RM instability-like evolution that is observed the higher the mixedness as time advances. However, in Fig .3.3 each case shows an initial decreasing mixedness. Just after the passage of the shock wave the interface exhibits large scale mixing, or entrainment, due to compression. However, as vorticity becomes active on the interface a traditional RM instability will experience greater small scale mixing and the mixedness will trend towards. Conversely, more suppressed cases which do not experience small scale mixing may either exhibit a shallow positive slope or stagnant mixedness behavior. Therefore, $M 1.7 B 0$, or the traditional RM instability, should exhibit the highest mixedness. From Fig. 3.3 this is indeed the case. It follows then that since $M 1.7 B_{\|} 50$ is more similar to $M 1.7 B 0$ it would have the second highest mixedness while $M 1.7 B_{\perp} 50$ has the least. From Fig. 3.2 it was expected that the mixing had stopped for $M 1.7 B_{\perp} 50$ by $\tau \approx 7$ with only an interface inversion occuring between $\tau \approx 7$ and $\tau \approx 15$; indeed the mixedness for this case is stagnant. The authors would like to note that while mixedness is a quantity sensitive to grid resolution, all simulations presented in this work share a resolution of 100 


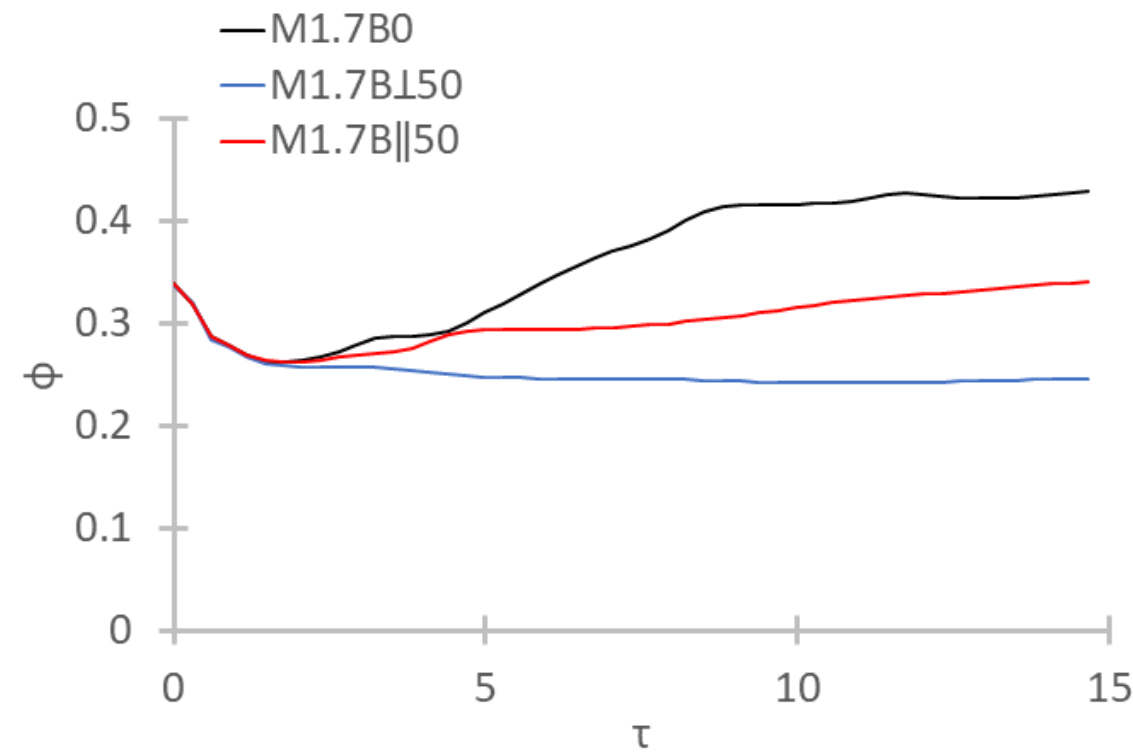

Figure 3.3: Mixedness, $\Xi$, with respect to $\tau$ for the magnetic orientation study.

nodes per diameter. Therefore, while mixedness may scale with the resolution the general trends are expected to hold so long as each simulation is compared on a fair grid. It should also be noted that the authors do not expect to be able to converge integral measurements in a 2D geometry, and thus to get reliable results one should run 3D simulations. Thus, the mixedness plots should be considered for their trends only.

$$
\Phi=\frac{h}{h_{\text {hom }}}=1-\frac{\int_{-L}^{L} \overline{\Upsilon_{P}^{\prime} \Upsilon_{P}^{\prime}} d x}{\int_{-L}^{L} \overline{\Upsilon_{P}}\left(1-\overline{\Upsilon_{P}}\right) d x}
$$

In order to understand the observed differences in both qualitative and quantitative mixing between the cases, one can look at the mechanism responsible for the evolution of the RM instability; that is vorticity. The vorticity is calculated using Eq. 3.3. When considering the effects of MHD waves, it is important to visualize the vorticity and explore whether that vorticity is causing mixing. To generate RM instability mixing the vorticity must act on the gradient of species. Thus, a new pa- 
rameter was created by taking the magnitude of the species gradient and multiplying it by the magnitude of the in-plane vorticity, as shown in Eq. 3.4. This parameter, $\Xi$, is shown at late time in Fig. 3.4 along side $\omega$.

$$
\begin{aligned}
& \vec{\omega}=\nabla \times \vec{u} \\
& \Xi=\left|\nabla \Upsilon_{P}\right||\omega|
\end{aligned}
$$

In Fig. $3.4 \omega$ is shown at times $\tau \approx 3,7$, and 15 while $\Xi$ is shown only at late time. For $M 1.7 B 0$ the vorticity is very neatly organized with similar behavior to the corresponding species pseudo-color. It was previously discussed that the maximum vorticity will be deposited at two sites normal to the shock wave direction. Its also known that the vorticity will be advected by the MHD waves along the field lines. $M 1.7 B_{\|} 50$ shows this through a stretched vortex field. The $M 1.7 B_{\|} 50$ case has a similar vorticity field to $M 1.7 B 0$; that is two vortices at $\pm \pi / 2$. However, the vorticity has been stretched and organized into layers as opposed to cores and can be seen to be acting over a larger area than the interface. The vorticity only appreciably intersects the interface on the outside perimeter of the leading edge near the tear drop like spikes. This stretched, less organized vortex field can explain both the stretching of the interface and the reduction in mixing.

The vorticity field in $M 1.7 B_{\|} 50$ exhibits a stretched field as predicted by previous discussion, thus one might expect the perpendicular magnetic field to move the vorticity normal to the shock wave direction in $M 1.7 B_{\perp} 50$ causing vortex competition. This would not only remove the vorticity from the interface faster than in $M 1.7 B_{\|} 50$, which advects tangentially in such a way as to interact with the interface over a longer time, but it could cause vorticity to compete within the interface and 
potentially cancel. While vortex competition may also occur in a parallel field case, the lack of vortex stretching in the direction of the shock wave propogation in the perpendicular field may lead to greater vortex cancellation. In $M 1.7 B_{\perp} 50$ the signs of vorticity have switched, that is the positive vorticity is on the right of the interface and the negative on the left, suggesting vortex advection across the interface. The vorticity also appears to be weaker than in the other two cases, also suggesting some mechanism to reduce the total vorticity.

In an effort to illuminate the effects of the vorticity advection $\Xi$ visualizes the interaction of vorticity and the species gradient, which is the source of mixing, and is shown in the second row of Fig. 3.4. $M 1.7 B 0$ shows a $\Xi$ field very similar to the vorticity and species. There is a strong $\Xi$ core where the plasma mass fraction approaches one and the vorticity is the strongest, and layers of $\Xi$ where vorticity works to mix the interface with the surrounding species; driving the species gradient to decay. The results of the stretched vorticity field in $M 1.7 B_{\|} 50$ can also be seen in $\Xi$ to cause mixing in the outer leading edges of the rollup structures. Here moderate vorticity acts on a very strong species gradient creating $\Xi$ twice the magnitude experienced in $M 1.7 B 0$. Despite its strength, $\Xi$ only works on the outer edge of the interface, causing the interface to stretch but not mix to levels seen in $M 1.7 B 0$.

In $M 1.7 B_{\perp} 50, \Xi$ is a full order of magnitude smaller than the other cases. Here, despite there being a large species gradient, that is almost no mixing has occurred, creating a large area with a steep species gradient. There is also almost no vorticity acting on the interface. In fact, the majority of the $\Xi$ which can be seen in this case appears at the leading and trailing edges of the interface, the areas where the inversion is most apparent. This also explains why the vortex features do not exist in this case; there is no vorticity acting on the edges of the interface where the vortices would form. 


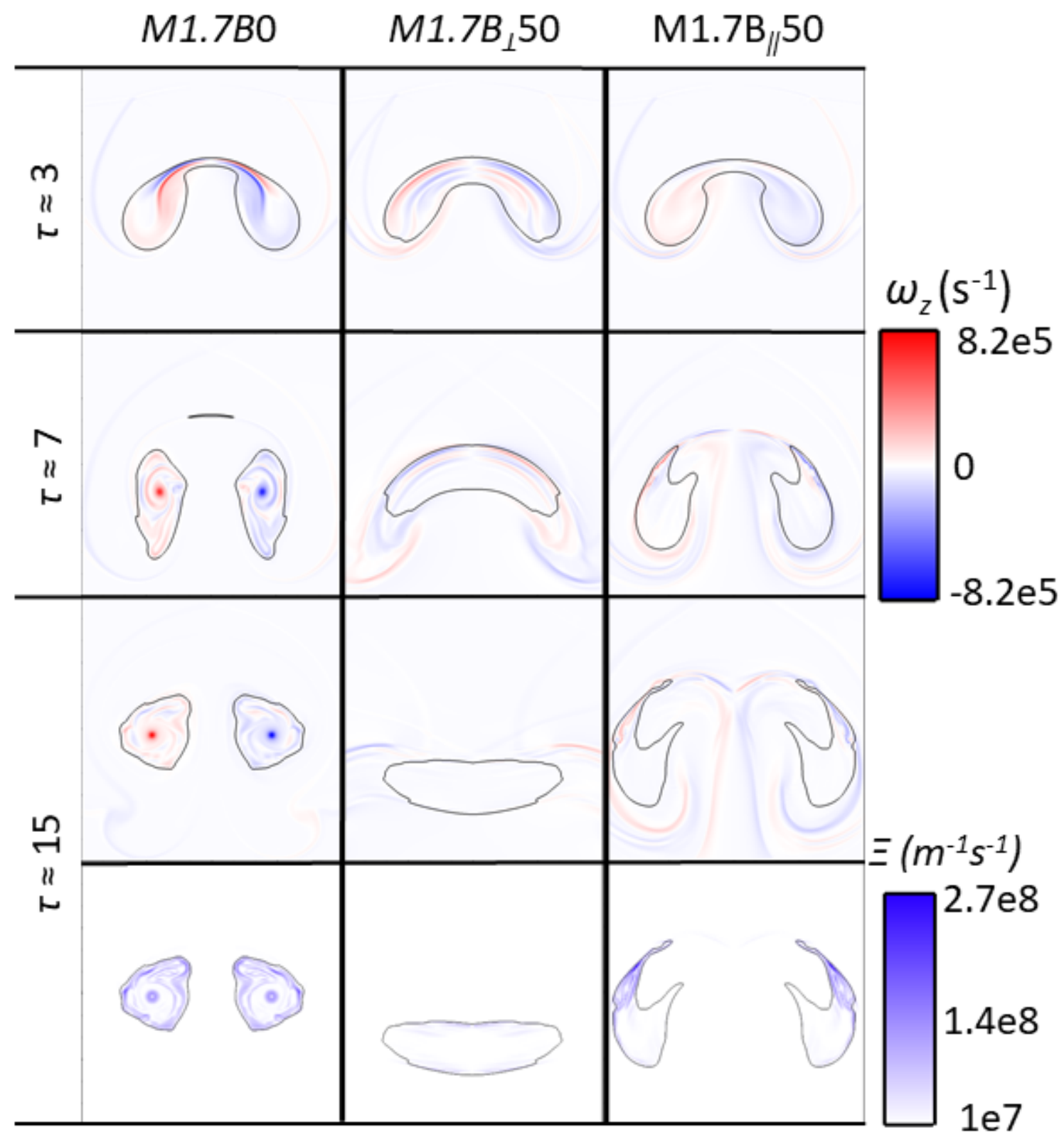

Figure 3.4: Late time pseudo-colors for $\omega$ and $\Xi$ for the magnetic orientation study. The images are centered about $y$, respective to $\tau$ at $y(\tau \approx 3)=3.11 \mathrm{~cm}, y(\tau \approx 7)=$ $7.64 \mathrm{~cm}$, and $y(\tau \approx 15)=15.55 \mathrm{~cm}$. 
To quantify the energy in the vorticity enstrophy is used, calculated as shown in Eq. 3.5. Enstrophy is typically integrated across the entire simulation domain, as the enstrophy exists at the interface for a RM instability and thus must be an accurate representation of the energy working to drive the instability. However, in MHD it has been shown that the vorticity advects, leaving the interface without mixing. As such, it is important to see what differences the area of integration can show for the enstrophy. This can be illuminated by Fig. 3.5. In this figure, two enstrophy values are plotted for $M 1.7 B 0, M 1.7 B_{\|} 50$, and $M 1.7 B_{\perp} 50$. The first enstrophy value, the local enstrophy, is denoted by the case name with a succeeding $R$. To calculate this value, the integral in Eq. 3.5 is taken across the same reduced area shown in images, a $5 \mathrm{~cm}$ by $5 \mathrm{~cm}$ square centered about the interface. The other quantity, labeled with a $T$ implies the traditional enstrophy taken across the entire domain.

Comparing the reduced area enstrophy between cases, it can be seen that $M 1.7 B 0$ has the most enstrophy, which can be expected as the traditional RM instability exhibits the most mixing and the highest vorticity. $M 1.7 B_{\|} 50$ has less enstrophy in early time but this steadily increases until it reaches similar levels to $M 1.7 B 0$. Similar to the previous discussion, $M 1.7 B_{\perp} 50$ shows an overall net decrease through time when compared to the other two cases. By late time the local enstrophy tends to low levels as the vorticity is carried away from the interface. Since this is a large reduction in local enstrophy it becomes necessary to consider the total enstrophy to see if the difference between the cases is due to the reduced area or the MHD effects. Here, it can be seen that the $M 1.7 B 0$ and $M 1.7 B_{\|} 50$ cases have similar enstrophy when compared to their reduced area values. In fact, it becomes difficult to see any difference for $M 1.7 B 0$ which is to be expected as there is no magnetic field to carry the vorticity away. In the $M 1.7 B_{\perp} 50$ case some of the lost local enstrophy is recovered by integrating across the entire domain, but a significant reduction in total enstrophy 
is observed compared to $M 1.7 B 0$ and $M 1.7 B_{\|} 50$. Both MHD RM instability cases exhibit less total enstrophy than the RM instability suggesting that the MHD waves cause vortex competition with the greatest effect in $M 1.7 B_{\perp} 50$ due to the normal direction of the Alfven velocity.

The magnitudes of integral mixing measurements, such as mixedness and enstrophy, are grid dependent and are difficult to achieve convergence with a limited resolution [104]. Previous work using a similar ALE code has shown that a resolution of over 1400 zones/diameter is necessary for convergence of enstrophy in inviscid simulations of a shock-cylinder problem like that presented here [105]. Despite this requirement for high resolition simulations and experimental comparisons have shown that even low resolution studies capture the morphology of the interface well and reproduce the effect of changing the Atwood number [106]. In addition, 2D simulations show similar results to 3D simulations for ealy time but they over predict vorticity by approximately $10 \%$ at late times [107].

$$
\epsilon=\int \vec{\omega} \vec{\omega} d A
$$

In effort to explain why the interface mixing has differed from the traditional RM instability in the MHD cases the vorticity has been studied. However, the movement of the vorticity, highlighted by the reduced area enstrophy, can offer a complicated story to consider. In an effort to capture the relationship between the species gradient and vorticity, $\Xi$ was used to give a qualitative metric at a single time in Fig. 3.4. To try to quantify this over time a correlation coefficient was created to relate the species gradient and vorticity as shown in Eq. 3.6. In this equation, the numerator is similar to $\Xi$ and is in fact the origin of this parameter.

The correlation coefficient relating the species gradient and vorticity, here out 


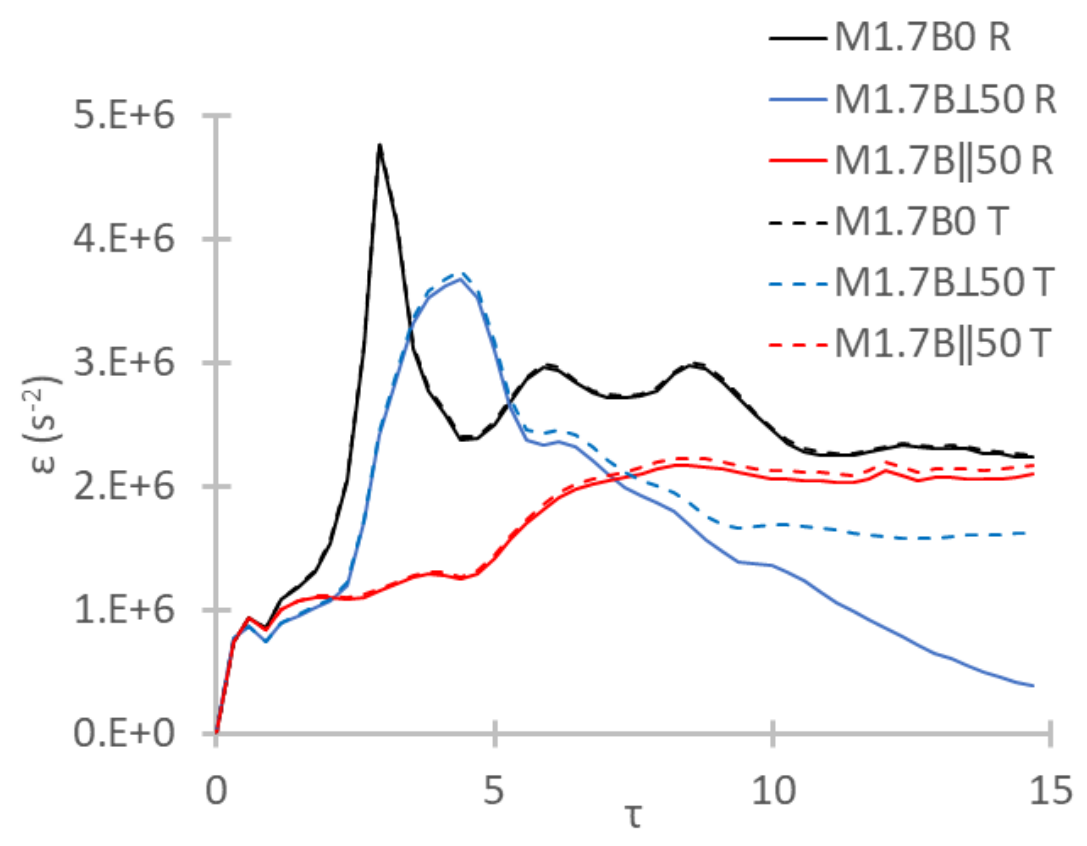

Figure 3.5: The total and reduced area (local) enstrophy for the $M 1.7 B 0, M 1.7 B_{\perp} 5$, and $M 1.7 B_{\|} 5$ cases.

referred to simply as the correlation coefficient, is plotted in Fig. 3.6. M1.7B0 exhibits the highest correlation between vorticity and the species gradient throughout time. However, this correlation decreases with respect to time. This is due to the nature of mixing, as vorticity acts on the interface it breaks down the species gradient and thus tends towards a lower correlation. The correlation for $M 1.7 B_{\|} 50$ also shows similar behavior to $M 1.7 B 0$ although less correlated through time. As opposed to mixing, the decrease of the correlation coefficient for $M 1.7 B_{\|} 50$ can be attributed to the vorticity advection away from the interface. Here, the vorticity acts to elongate the interface but doesn't cause mixing every where. $M 1.7 B_{\perp} 50$ shows significantly less correlation at late time than the other cases. This is due to the vorticity motion along the magnetic field lines rather than mixing, which drops the correlation coefficient to a value lower than 0.1 . 


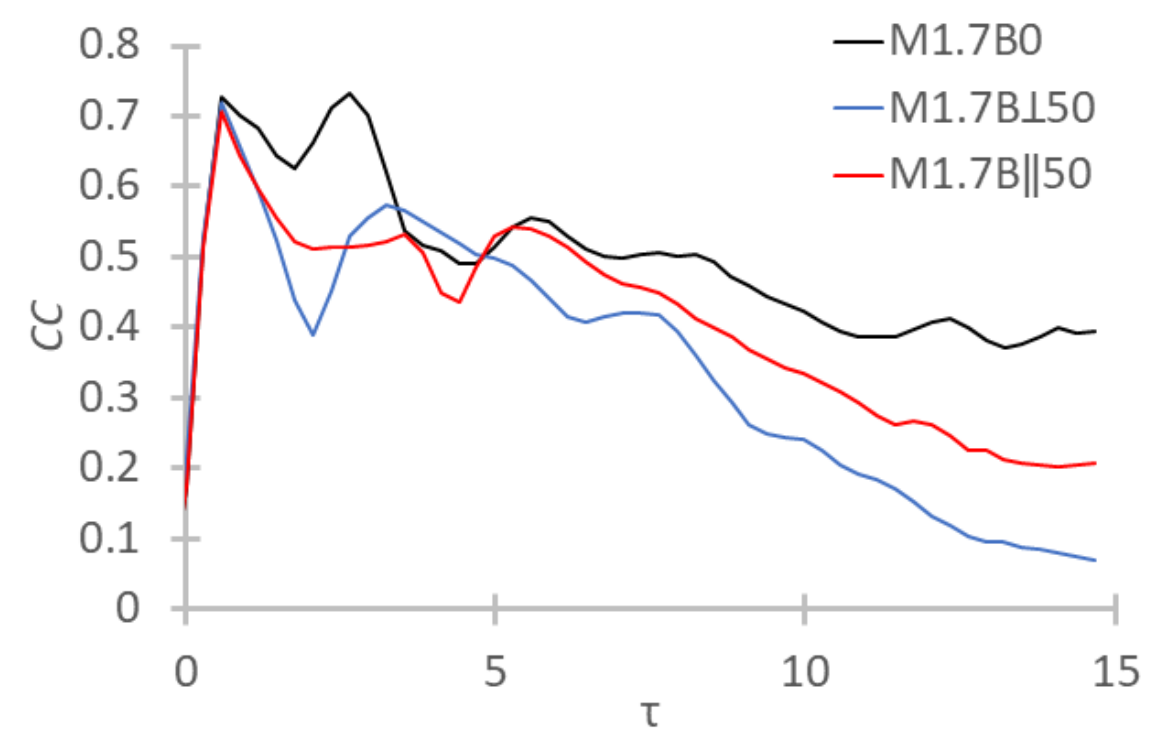

Figure 3.6: The correlation coefficient with respect to $\tau$ for the magnetic orientation study.

$$
C C=\frac{\left\langle\left|\nabla \Upsilon_{P}\right||\omega|\right\rangle}{\sqrt{\left\langle\left|\nabla \Upsilon_{P}\right|^{2}\right\rangle\left\langle|\omega|^{2}\right\rangle}}
$$

The following sections will only consider the species and vorticity pseudo-colors qualitatively and the correlation coefficient and the mixedness plots quantitatively. Through the observation of these parameters one can infer the others. For instance, along with the knowledge presented in this section, one can infer the interaction of the vorticity with the species gradient, $\Xi$, by observing the mixedness and the vorticity field. If the mixedness increases and there is vorticity present at the interface, then it would be expected $\Xi$ would show the interaction of the species gradient and the vorticity. The enstrophy in Fig. 3.5 was used to show that the energy in the vorticity decreases in the presence of a magnetic field, and that one can quantify the energy of the vorticity acting on the interface by considering a small area about the interface. However, when considering the species psuedo-color and the mixedness, one can infer the species gradient behavior through time. Thus, any differences between 
the mixedness and the correlation coefficient must be due to the behavior of the vorticity, which can be shown via the vorticity psuedo-color.

\subsubsection{Magnetic Field Strength}

The previous section showed that both the presence of a magnetic field and the orientation of said field effects the evolution of the MHD RM instability. Therefore, it is of interest to vary the strength of the initialized magnetic field, which controls the dispersion of the MHD waves via the Alfven velocity. Thus, this section includes both the parallel and perpendicular magnetic configurations and varies them from 100, 250, and 500 G. Figure 3.7 shows species pseudo-color plots for the parallel field configuration. It is expected that the weaker the magnetic field, the more similar to the traditional RM instability the instability will be. The weaker magnetic fields will remove vorticity from the interface at a slower rate allowing more time for the baroclinic vorticity to cause mixing. It was previously shown that at early times $M 1.7 B_{\|} 50$ was similar to $M 1.7 B 0$ it follows that both $M 1.7 B_{\|} 10$ and $M 1.7 B_{\|} 25$ will

exhibit vortex-like features. In fact, at $\tau \approx 3$ these two cases look very similar to $M 1.7 B 0$. However, by mid-time the field strength effects become noticeable. Here, $M 1.7 B_{\|} 10$ shows similar organization to the traditional RM instability but with a much tighter interface and no spike-like structure emerging on the leading edge. This organization continues through late time with tight vortices that have more pure plasma species than the traditional case. Thus, while $M 1.7 B_{\|} 10$ is very similar to $M 1.7 B 0$ it still has reduced mixing.

$M 1.7 B_{\|} 25$ shows a similar instability to $M 1.7 B_{\|} 50$, mid-time tear drop like spikes are developed. However, in $M 1.7 B_{\|} 25$ these spikes are on the inner leading edge. This trend continues into late time with the elongation of these spikes and the preservation 
of the plasma species. That is, as the parallel magnetic field strength is increased, the RM instability mixing is further decreased.

Tabling the discussion on the parallel field for a moment, consider Fig. 3.8. This figure contains a similar study as Fig. 3.7 but for the perpendicular field. Similar to the parallel field study, the early time weaker magnetic field cases show stronger similarities to $M 1.7 B 0$. That is, $M 1.7 B_{\perp} 10$ and $M 1.7 B_{\perp} 25$ both have clear vortex features and a thin bubble front. However, with increasing magnetic field strength the vortices are reduced in size and the bubble front is thickened. Unlike $M 1.7 B_{\|} 10$, $M 1.7 B_{\perp} 10$ shows significant departure from the RM instability by mid-time. Here $M 1.7 B_{\perp} 10$ shows flattened vortex structures connected by a diffusive bubble front, a component which is negligible in $M 1.7 B 0$. At late time, in $M 1.7 B_{\perp} 10$ the bubble front prevails, albeit stretched and diffusive. The vortex structures have thinned as mixing has worked to diffuse the plasma species, but the morphology and mixing is significantly different even in the presence of this weak perpendicular magnetic field. This evolution is as different from the traditional RM instability as the evolution in $M 1.7 B_{\perp} 50$. However, instead of obvious suppression this interface appears very diffusive and stretched. Qualitatively it is difficult to claim that $M 1.7 B_{\perp} 10$ is suppressed compared to $M 1.7 B 0$, although it is clearly effected by the magnetic field.

$M 1.7 B_{\perp} 25$ is more similar to $M 1.7 B_{\perp} 50$ exhibiting a strong bubble front at midtime. Unlike $M 1.7 B_{\perp} 50 M 1.7 B_{\perp} 25$ has significant curvature, which resembles the mushroom shape in $M 1.7 B_{\perp} 10$. The vortices which were present at early time have almost vanished, with thin finger-like structures shown peeling back at mid-time. At late time these structures are gone and only a sharp point remains on either side of the interface. At this time $M 1.7 B_{\perp} 25$ has a significant reduction in mixing from $M 1.7 B_{\perp} 10$ though more curvature than $M 1.7 B_{\perp} 50$. While $M 1.7 B_{\perp} 25$ has not inverted it's leading and trailing edges, the finger-like structures which formed in 


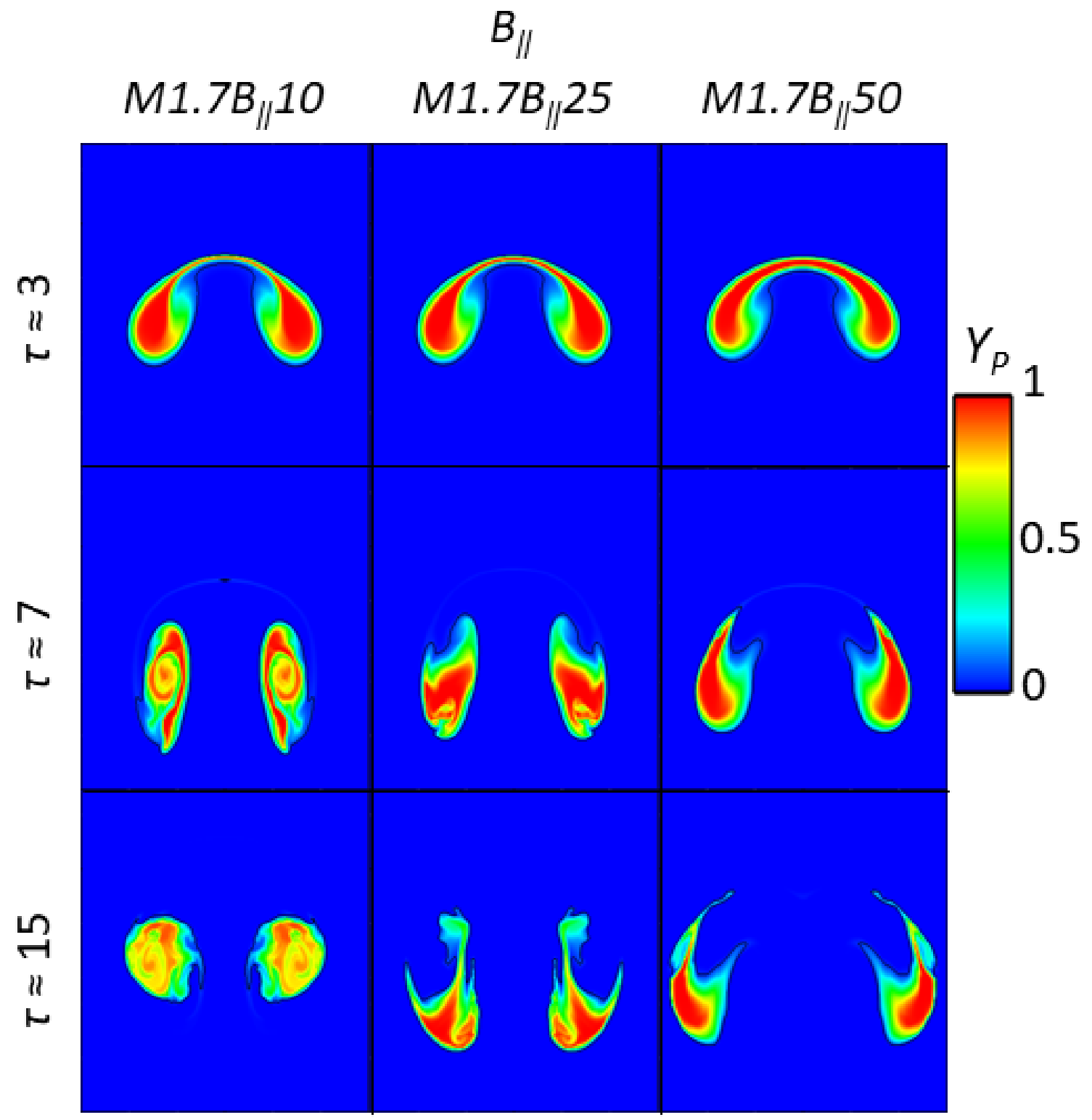

Figure 3.7: Magnetic strength study; Pseudo-color of species for the parallel magnetic field cases with respect to $\tau$. The images are centered about $y$, respective to $\tau$ at $y(\tau \approx 3)=3.11 \mathrm{~cm}, y(\tau \approx 7)=7.64 \mathrm{~cm}$, and $y(\tau \approx 15)=15.55 \mathrm{~cm}$. 
place of vortices have seemingly been inverted. This is likely the same mechanism that works to invert the interface in $M 1.7 B_{\perp} 50$, albeit weaker due to the weaker magnetic field.

Figure 3.9 shows the mixedness for the magnetic field strength study. Similar to the qualitative mixing in Figure $3.7, M 1.7 B_{\|} 10$ shows the most similar mixedness to $M 1.7 B 0$. Both cases steady out around $\tau \approx 7$, however $M 1.7 B_{\|} 10$ shows a reduction throughout time. Interestingly the 250 and $500 \mathrm{G}$ fields exhibit similar mixedness history for the parallel and perpendicular fields respectively. This indicates that while increasing the strength of a magnetic field may change the morphology of the species and vorticity fields, there may be a limit to its effect on mixing. That is, the MHD waves are a function of the Alfven velocity, which in turn is a function of the magnetic field strength. Despite any macroscopic changes to the interface, the mixedness suggests that the maximum suppression, within the range of these magnetic field strengths, can happen as low as $250 \mathrm{G}$ for either field orientation with no additional effect made apparent by doubling the magnetic field strength.

Perhaps the most interesting case in this section is $M 1.7 B_{\perp} 10$. Figure 3.8 shows a significantly stretched interface throughout time. This is the only case in which a thin bubble front connects to vortex features at late times. Qualitatively it's difficult to know if the regions where the plasma species fraction is more concentrated have a greater area than other cases, but the mixedness seems to suggest that $M 1.7 B_{\perp} 10$ has superior mixing even compared to the traditional RM instability. This is because the vortex field is stretched and weakened by the magnetic field, stretching the interface without breaking down the bubble front or organizing vortices fully. This creates a larger mixing surface area, thus leading to greater mixedness, or a larger ratio of mixing to entrainment. This condition may be temporary, as the bubble front separates with time, leading to a mixedness more similar to the other cases at late 


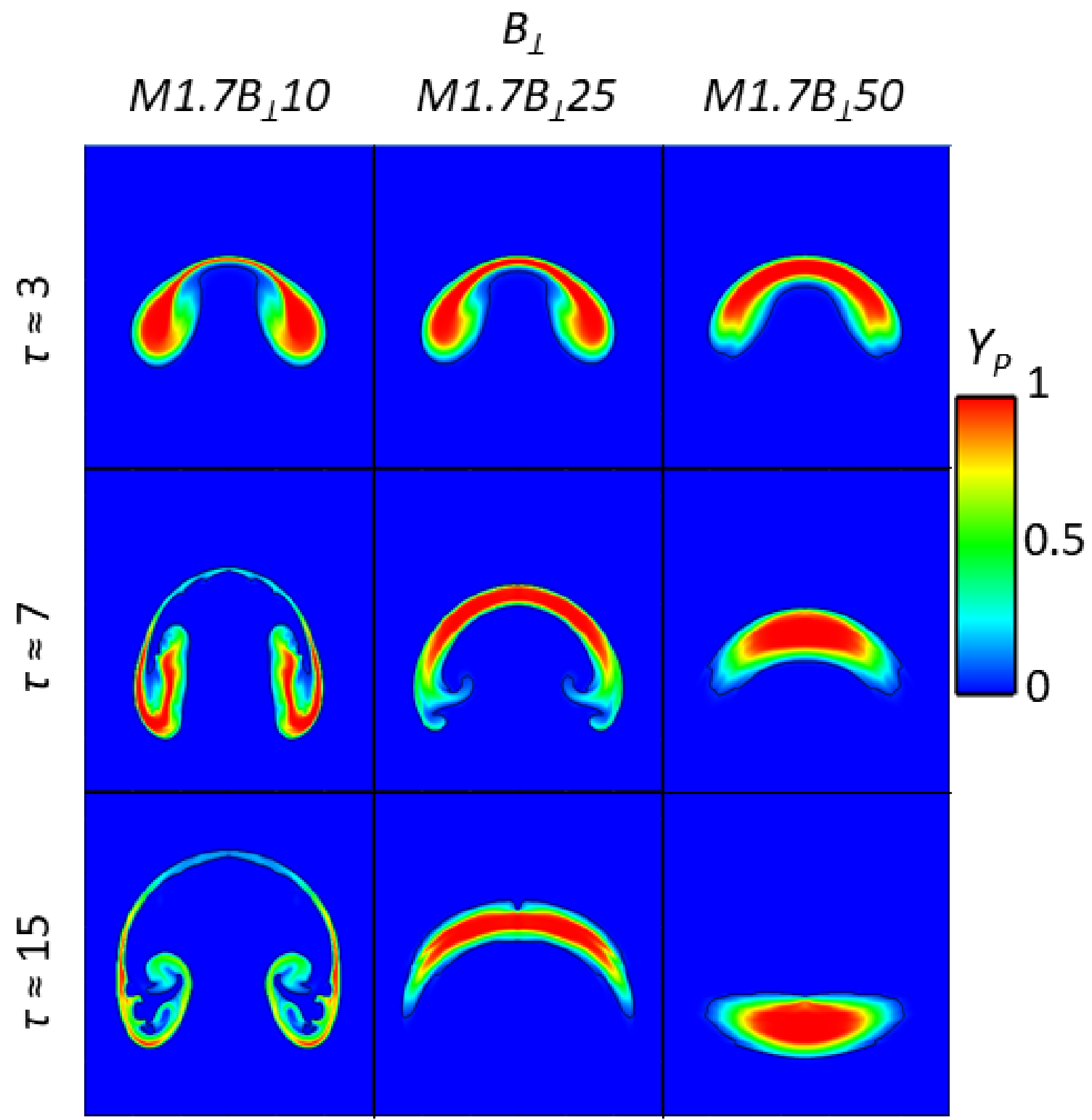

Figure 3.8: Magnetic strength study; pseudo-color of species for the perpendicular magnetic field cases with respect to $\tau$. The images are centered about $y$, respective to $\tau$ at $y(\tau \approx 3)=3.11 \mathrm{~cm}, y(\tau \approx 7)=7.64 \mathrm{~cm}$, and $y(\tau \approx 15)=15.55 \mathrm{~cm}$. 


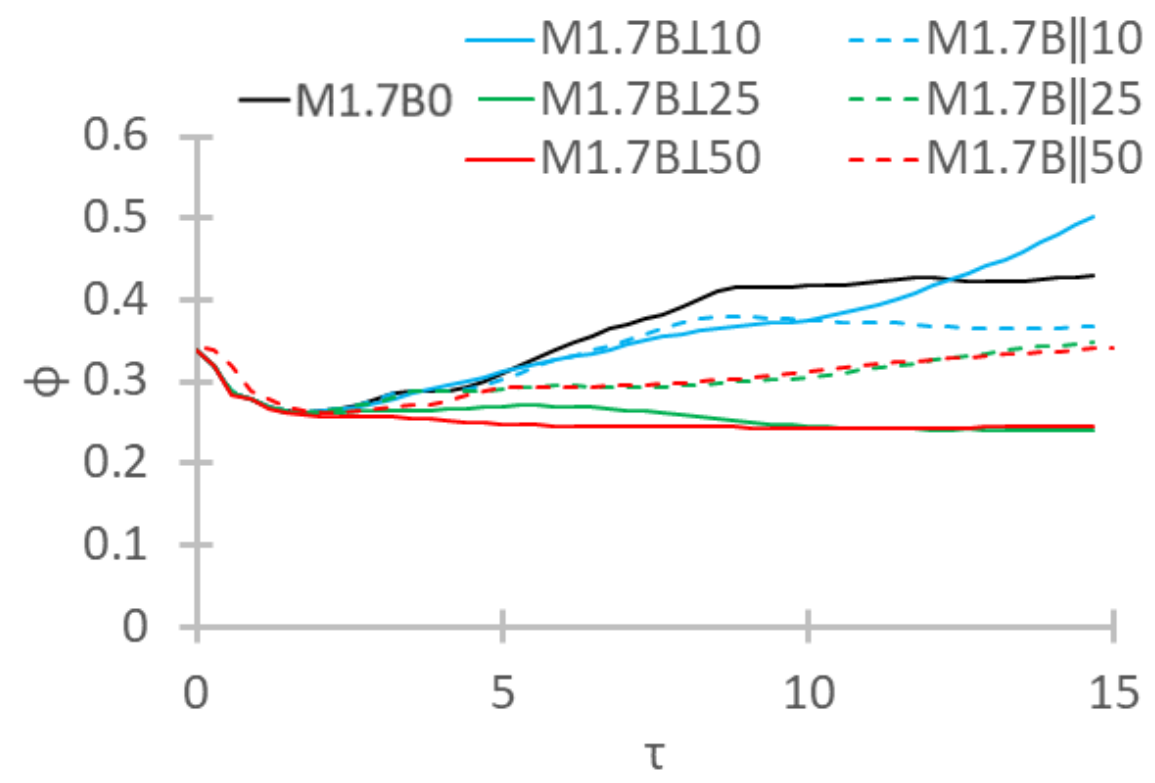

Figure 3.9: The mixedness for the magnetic strength study. Here $B 0$ is included to highlight the effects of the magnetic field.

times.

To understand the qualitative and quantitative mixing differences in figures 3.7, 3.8, and 3.9; the late time vorticity field is shown in Fig. 3.10. For both fields, the organization of the vortex field increasingly breaks down with stronger field strengths. As discussed earlier, this is because the vorticity is being carried away from the interface along the magnetic field lines eventually causing vortex competition. For the parallel cases, the vorticity field takes on a mushroom shape with strong organized vortex cores. For $M 1.7 B_{\|} 10$ the magnetic field is weak enough that the majority of the vorticity is not removed from the interface and thus causes RM instability mixing. As the magnetic field strength increases, the vorticity advects further away from the interface. In $M 1.7 B_{\|} 25$ the organized vorticity seems to form more sheet-like structures and is pushed into the stem region causing the inner teardrop like spikes to form. This vorticity is moved further away in $M 1.7 B_{\|} 50$. Evidence of the inner spike can be seen more clearly here, however as the vorticity is pushed away further 


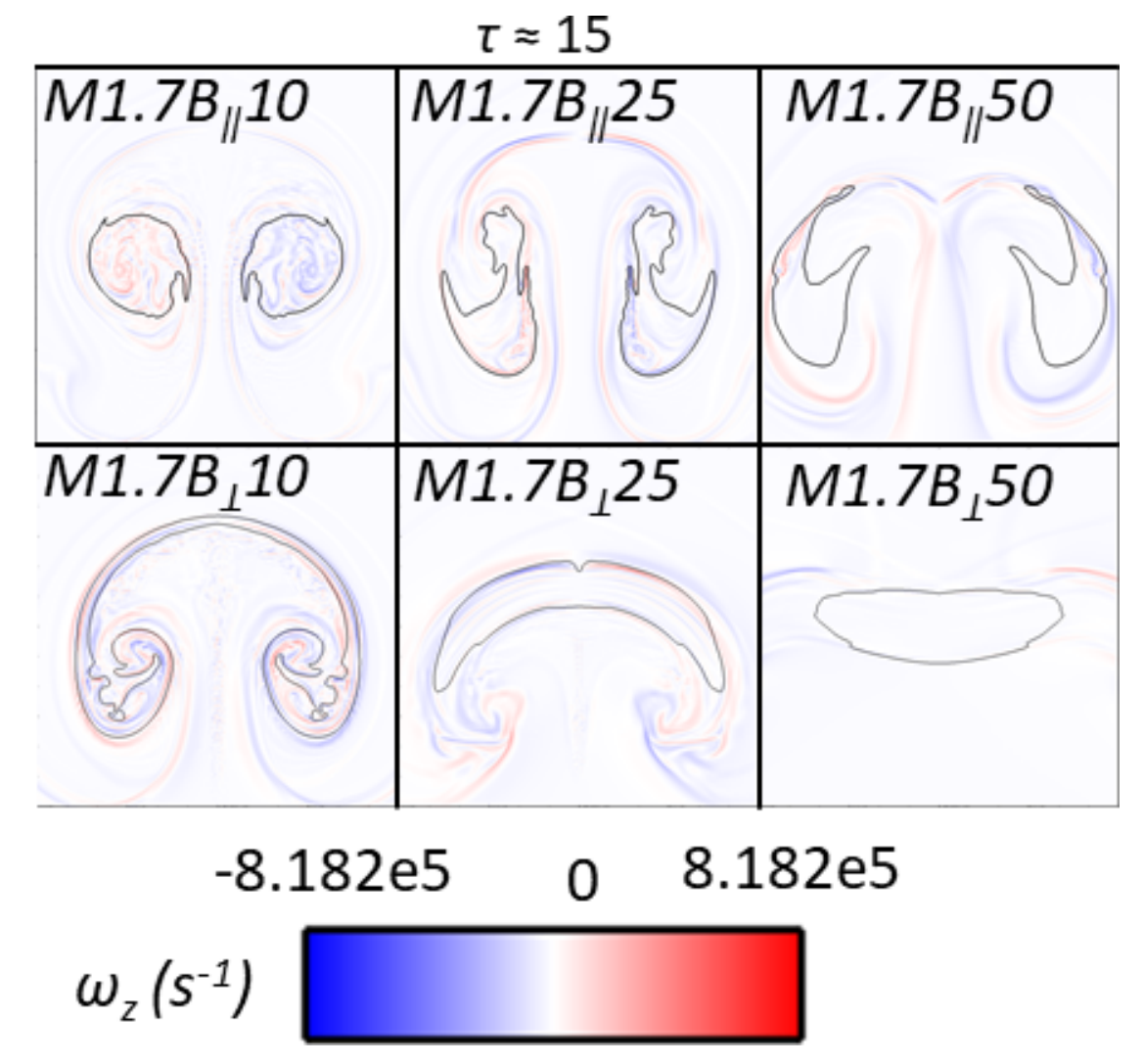

Figure 3.10: Late time vorticity field for the magnetic strength study. The images are centered about $y(\tau \approx 15)=15.55 \mathrm{~cm}$.

to the outside of the interface, the outer leading-edge spike is more prevalent.

The mushroom shape of the vorticity field, noticeable in the parallel cases, is also apparent in the weak perpendicular cases. For $M 1.7 B_{\perp} 10$ the field is very similar to $M 1.7 B_{\|} 10$. However, here the vorticity movement is clearly more normal to the direction causing the vorticity to create a large diffusive interface which may indicate more mixing. The vorticity is also less organized with more visible layers. Comparing these two cases, it is also noticeable that $M 1.7 B_{\perp} 10$ has more positive and negative vorticity interaction than in $M 1.7 B_{\|} 10$, which has more organized strong positive vorticity on the left-hand side and negative on the right. In $M 1.7 B_{\perp} 25$ the mushroom shape vorticity field becomes stunted in the direction of shock transit, with the 
vorticity being pushed to the side by the perpendicular magnetic field. Here, more negative vorticity can be seen on the left, while stronger positive vorticity is apparent on the right having already alternated sides due to the traverse MHD waves. In this case a complex interaction of positive and negative vorticity can be seen near the bottom side of the interface. Earlier in time, these spike structures appeared like roll-ups which eventually took finger-like shapes at mid-time (see $\tau \approx 7$ Fig. 3.8) and have disappeared by late time. It's likely this vortex interaction is responsible for the apparent reduction in organization of this structure, and that this same interaction is responsible for the late time inversion of the $M 1.7 B_{\perp} 50$ case.

The correlation coefficient for the magnetic field study is presented in Fig. 3.11. Similarly to what was observed in Fig. $3.9, M 1.7 B_{\perp} 10$ has a higher correlation due to the stretched species and vorticity fields. That is, the species gradient acts over a larger area and at a greater magnitude than $M 1.7 B 0$, driving up both mixedness and the correlation between the fields. Aside from $M 1.7 B_{\perp} 10$, it might be expected that M1.7B0 is the most correlated considering the absence of MHD effects. However, the correlation coefficient is dependent upon the magnitude of vorticity acting on the magnitude of the species gradient. Therefore, if a case were to exceed the correlation of the traditional RM instability it would need either greater vorticity at the interface or a larger, longer lasting species gradient. It has been shown explicitly that in the presence of a magnetic field the vorticity moves away from the interface in Section 3.2.1, and Fig. 3.10 shows that even a weak magnetic field can affect the vortex field. Therefore, the increased correlation in $M 1.7 B_{\perp} 10$ is due to the larger interface with steep species gradients.

$M 1.7 B_{\|} 10$ and $M 1.7 B_{\|} 25$ have a similar correlation to $M 1.7 B 0$. This is easy to explain for $M 1.7 B_{\|} 10$, which is the most similar case to $M 1.7 B 0$, both through qualitative mixing and in its vortex field. $M 1.7 B_{\|} 25$ gets its large correlation then 


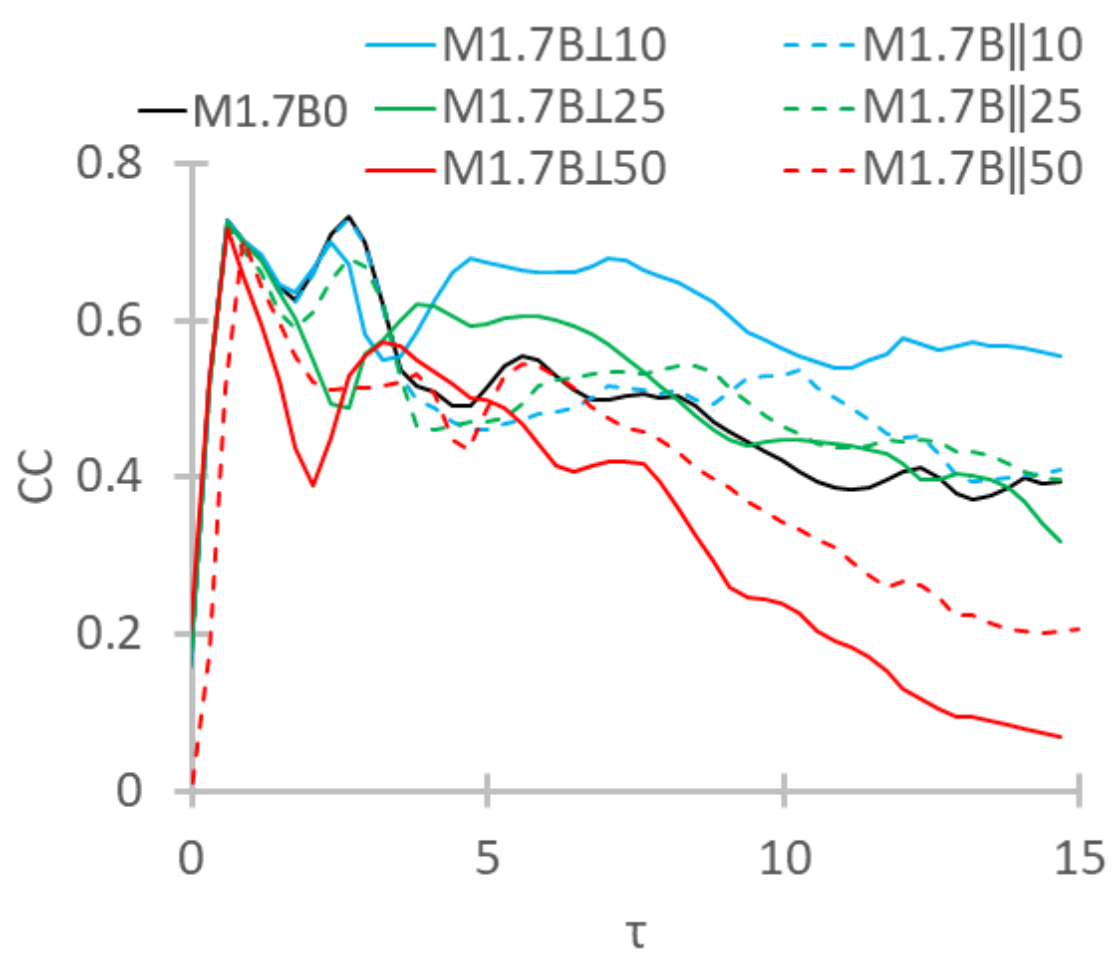

Figure 3.11: The correlation coefficient, given as Eq. 3.6, for the magnetic strength study

from its species gradient. Figure 3.8 shows a relatively unmixed stretched interface, with a large gradient of species in the inner leading-edge spikes. Looking at Fig. 3.10 the majority of the strong vorticity acting on the interface acts on this region, causing the mixedness seen in Fig. 3.9. Thus, the large correlation coefficient is due to the interaction of this large gradient with its vorticity, whereas the correlation coefficient for $M 1.7 B 0$ is due to the large vorticity acting on the species gradient. The remaining cases act as one may predict. That is, neglecting $M 1.7 B_{\perp} 10$, as the magnetic field strength is increased the correlation coefficient is decreased, and the perpendicular field cases exhibit less correlation than their parallel field counterparts. 


\subsubsection{Incident Shock Wave Strength}

In the previous section the magnetic field strengths and orientations were varied for the cylindrical MHD RM instability with $M=1.66$. This Mach number provides the pressure gradient necessary to deposit baroclinic vorticity. It is common to vary the Mach number in literature to understand how an interface might evolve due to different impulsive accelerations. This is important as Richtmyer's linear model says that the amplitude growth of the RM instability is proportional to the shock jump velocity [9], thus increasing the Mach number leads to a stronger instability. In addition to creating a stronger instability, the Mach number also determines postshock conditions that influence the evolution of the interface (e.g. density). In the MHD RM instability, the density also effect the MHD wave velocities. Thus, two additional Mach numbers will be studied here; $M=1.2$ and $M=2.2$. These Mach numbers were selected for their incremental increase in the shock jump velocity by $\approx 200 \mathrm{~ms}^{-1}$. This study is different from those presented in Sections 3.2.1 and 3.2.2 as it is not a study on MHD parameters alone, but rather on a traditional hydrodynamic variable which has MHD consequences. Both the classic RM instability, $B=0$, and a perpendicular magnetic field of 500 gauss, $B=B_{\perp}=500 \mathrm{G}$, will be presented for each Mach number. The perpendicular magnetic field was selected as it shows the greatest difference from the RM instability case.

Figure 3.12 shows the species and the vorticity pseudo-colors at late time, $\tau \approx 15$. The traditional RM instability cases, shown in the top row of Fig. 3.12, exhibit classic RM instability behavior which has been described in other works. Therefore, for brevity it will only be said that with increasing Mach number more baroclinic vorticity is deposited on the interface driving mixing at a faster rate. Hence $M 2.2 B 0$ evolves faster and exhibits more mixing and stronger vorticity than either $M 1.7 B 0$ or 
$M 1.2 B 0$. The cases all share similar organization in the species and vorticity fields.

Moving on to consider the MHD RM instability, the magnetic field cases are shown in the second row of Fig. 3.12. At late time $M 1.2 B_{\perp} 50$ shows a large very round interface with almost no mixing. Here, the interface has been slightly compressed but otherwise undisturbed. Its organization is very similar to the initial conditions (Fig. 3.1) with a thick, clear, and unmixed diffusion layer. Looking at its vortex field, one can see almost no vorticity near the surface of the interface, and indeed what vorticity is present in this image is low enough to be incomparable when considering the magnitude of vorticity in $M 1.2 B 0$. As the Mach number increases the MHD RM instability interfaces exhibit greater compression. In addition to this compression it appears as though the greater Mach number also leads to more mixing of the diffusion layer. For example, when considering $M 1.7 B_{\perp} 50$, the interface shows more compression than its low Mach number neighbor. The high Mach number case, $M 2.2 B_{\perp} 50$ shows a highly compressed interface with significantly less mixing and no resemblance to its magnetic field free counter-part, $M 2.2 B 0$. The vorticity field of both these cases is similar in that they exhibit negative and positive layers of vorticity on the left and right sides of the interface respectively and neither case appears to have any vorticity active on the interface.

To understand how this has occurred, one should remember the mechanisms which work to remove vorticity are a function of the Alfven velocity. While the magnetic field in these cases is initialized at $500 \mathrm{G}$, the Alfven velocity also has the density of the species in the denominator. Thus, there are two effects to consider for a larger Mach number. First off, the post shock density is proportional to the Mach number while the Alfven velocity is inversely proportional to the density. Since the magnetic field is also a function of the density of the perfect conductor, (Eq. 1.20), the Alfven velocity then goes as $\approx \rho^{1 / 2}$, meaning that $V_{A}$ then increases with increasing Mach 


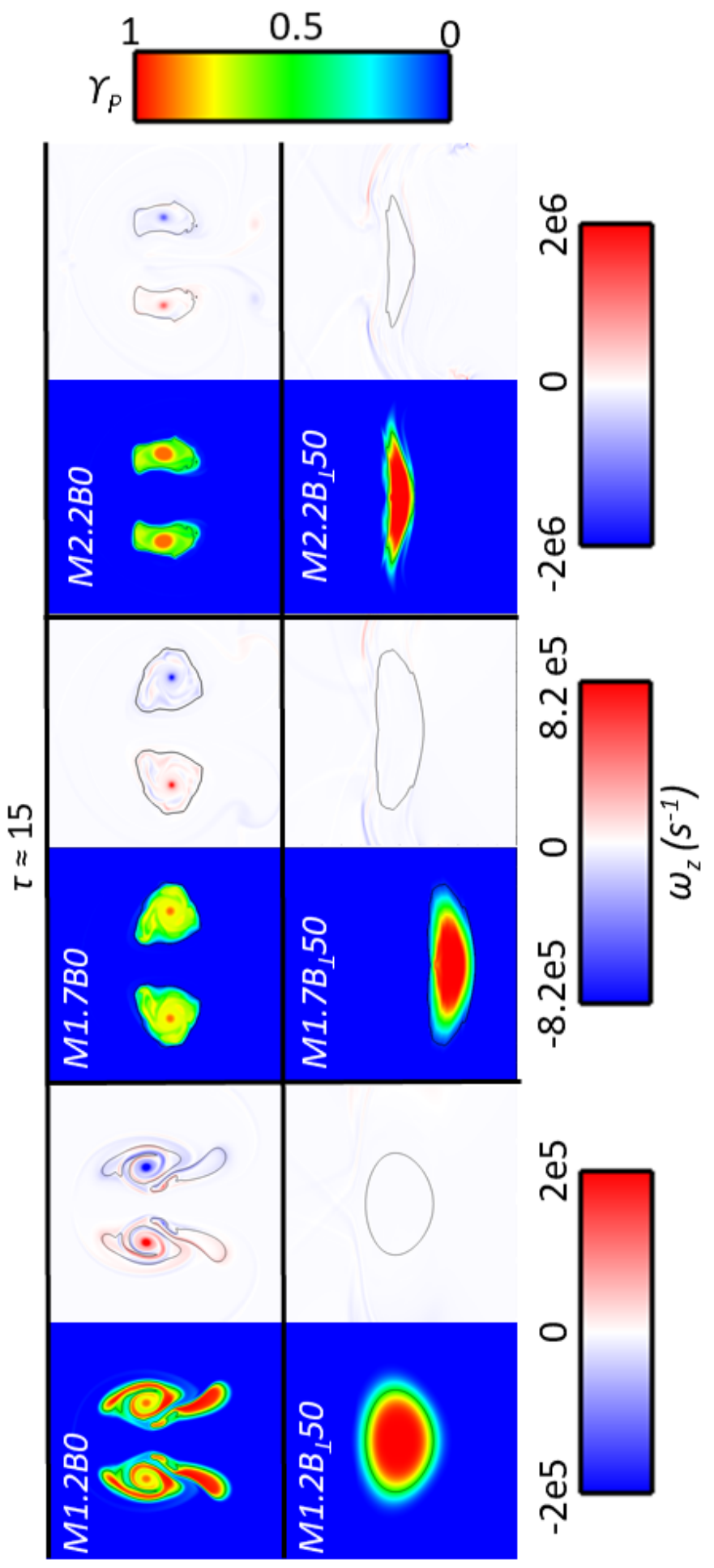

Figure 3.12: The late time species and vorticity plots for the incident shock wave strength study. The species pseudo-colors are all on the same scale shown on the right of the image while the vortices are Mach number specific and shown below each group. The images are centered about $y$, respective to $M$ at $y(M=1.2)=7.60 \mathrm{~cm}$, $y(M=1.7)=15.55 \mathrm{~cm}$, and $y(M=2.2) \overline{\overline{92}} 19.40 \mathrm{~cm}$. 
number. Of course the higher Mach number will also deposit a higher vorticity at the interface. Thus, higher Mach number cases will have stronger vorticity deposition that acts on the interface for a shorter amount of time.

Figure 3.13 shows the mixedness for the Mach number study. The non-magnetic cases (black lines) show greater mixing with increasing Mach number as expected for the RM instability. However, in the presence of a magnetic field the higher Mach numbers exhibit lower mixedness. It was observed that $M 2.2 B_{\perp} 50$ is more compressed and exhibits a smaller diffusion layer than the other cases. Again, the mixedness is the ratio of mixing to entrainment. The area of entrainment between the MHD RM instability cases is similar but as the stronger Mach number cases have greater Alfven velocity there is less time for the vorticity to act on them and cause mixing. This, paired with a smaller diffusion layer, leads to a reduced mixing width which in turn leads to less mixedness. Therefore, despite the observed interface compression in Fig. $3.12, M 2.2 B_{\perp} 50$ exhibits less mixedness than $M 1.7 B_{\perp} 50$ which in turn exhibits less then $M 1.2 B_{\perp} 50$.

Finally, Fig. 3.14 shows the correlation coefficient for the Mach number study. Considering only the traditional RM instability cases, it is expected that the higher the Mach number the lower the correlation due to mixing driving the species gradient to zero. This can be seen in Fig. 3.14, where $M 1.2 B 0$ has the highest correlation coefficient and $M 2.2 B 0$ has the lowest of the classic RM instability cases. For the MHD RM instability cases a increasing correlation is observed with increasing Mach number and the $M 1.2 B_{\perp} 50$ shows the smallest correlation, for all cases, at late time. From the qualitative evidence this makes sense, the vorticity deposited is simply too weak to cause mixing and doesn't remain near the interface thus driving the correlation coefficient down.

$M 2.2 B_{\perp} 5$ exhibits the greatest correlation of the MHD RM instability cases. This 


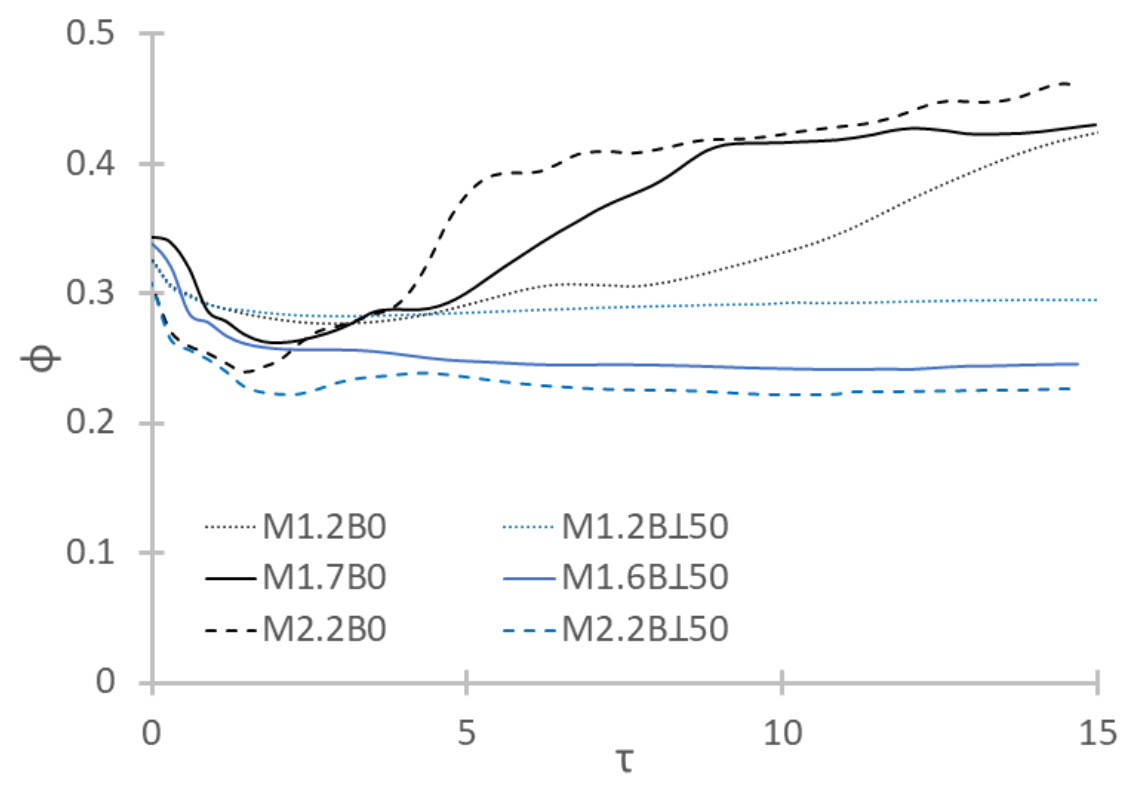

Figure 3.13: The mixedness for the incident shock wave study. Small dash lines indicate $M=1.22$, solid lines indicate $M=1.66$, and large dash lines indicate $M=2.2$; while black represents cases without a magnetic field and blue represents cases with a perpendicular magnetic field of $500 \mathrm{G}$.

is due to the large magnitude of vorticity deposited at the interface. The vorticity is advected via the MHD waves but acts over a larger area and at a greater magnitude everywhere than its weaker Mach number counterparts. Therefore, while stronger baroclinic vorticity is able to further compress the interface, it is also advected quicker over a greater area and at a higher magnitude. This can be seen by looking at the vortex fields for the MHD RM instability cases in Fig. 3.12. M2.2 $B_{\perp} 50$ exhibits the largest vortex field at the highest magnitude despite its higher vortex advection. Similarly, the vortex field in $M 1.7 B_{\perp} 50$ covers a larger area at a stronger magnitude than $M .12 B_{\perp} 50$ but is still weaker than $M 2.2 B_{\perp} 50$. Thus, despite there being little to no vorticity acting on the interface in any of the MHD RM instability cases, there is increasingly more with higher Mach numbers leading to an increased correlation coefficient. However, each of the MHD RM instability cases exhibit far less correlation 


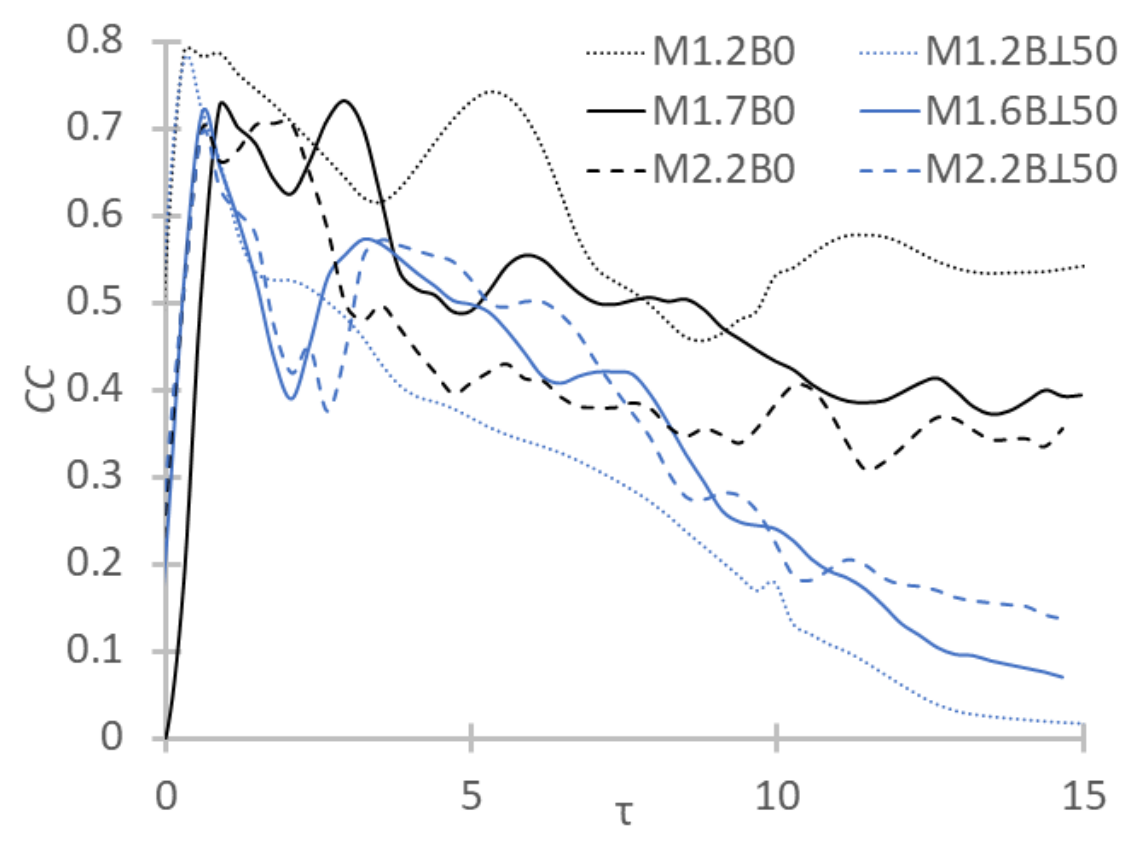

Figure 3.14: The correlation coefficients for the incident shock wave study. Small dash lines indicate $M=1.22$, solid lines indicate $M=1.66$, and large dash lines indicate $M=2.2$; while black represents cases without a magnetic field and blue represents cases with a perpendicular magnetic field of $500 \mathrm{G}$.

than their classic RM instability counterparts.

\subsection{Conclusions}

This chapter studied the cylindrical MHD RM instability by varying the magnetic field orientation, magnetic field strength, and the incident shock wave Mach number (acceleration strength). The cylindrical interface was selected as its evolution is independent of the orientation of either the shock wave or the magnetic field direction. Instead, the evolution depends only on the orientation of the shock transit direction relative to the magnetic field. The magnetic field parameters were selected to explore their effects on vorticity advection caused by MHD waves, while the Mach number was selected to explore how increasing baroclinic vorticity deposition may effect the 
growth of the MHD RM instability. It was shown that interface mixing is a function of the amount of vorticity deposition, the rate at which vorticity is advected, and the advection direction via Alfven waves. This leads to a rich parameter space where some counterintuitive results are found in mixing measures. To understand the parameter space the simulations were observed qualitatively via species and vorticity fields and quantitatively through mixedness, enstrophy, and the correlation coefficient of the vorticity and species gradient. It was found that in general the presence of the magnetic field suppresses RM instability-like evolution by the advection of vorticity, with a stronger field, and preferential field orientation resulting in greater suppression. Overall, it was shown that interface mixing is a function of the amount of vorticity deposition, the rate at which it is advected, and the direction it is advected by Alfven waves.

Expanding upon results found in literature, it was shown here that the presence of either a perpendicular or parallel magnetic field in the MHD RM instability suppresses RM instability evolution; with a perpendicular magnetic field showing less mixedness and significantly reduced RM instability morphology compared its parallel field counterpart. This is because in the presence of a magnetic field the whole of the deposited baroclinic vorticity, which drives the evolution in the RM instability, no longer remains on the density gradient. This interaction, shown by the speciesgradient-vorticity correlation, can be seen to be significantly reduced for the MHD RM instability cases when compared to the traditional RM instability. The differences in field orientation are further highlighted by the vorticity field and enstrophy. The vorticity is stretched significantly in the direction of the magnetic field, hence the parallel field stretches the vorticity along the shock wave direction. Enstrophy versus time curves, for all enstrophy and enstrophy only near the interface show that both orientations result in lower total enstrophy but that the perpendicular orienta- 
tion has much lower enstrophy near the interface. Furthermore, vorticity is able to traverse the interface rapidly in the perpendicular field orientation, allowing positive and negative vorticity from opposite sides of the interface to interact and compete, diminishing the enstrophy.

As the magnetic fields are the driving mechanism for the vorticity transport it follows then that as the magnetic field strength increases so does the suppression of the RM instability evolution. However, by examining magnetic fields of 100, 250, and $500 \mathrm{G}$ in both orientations two nonobvious effects were observed. First, there appears to be some limit to the effectiveness of the magnetic field on mixing. That is, for both field orientations increasing the magnetic field from 250 to $500 \mathrm{G}$ did not significantly affect the mixedness. However, the vorticity and species fields displayed differences in morphology. Secondly, a competing effect was observed for the perpendicular field of $100 \mathrm{G}$. Here the magnetic field was unable to remove the vorticity from the interface but instead moved it in such a way as to cause significant morphological differences. This interface appears much more diffusive and exhibits higher mixedness and correlation than any other case with $M=1.66$. This implies that while strong fields have been shown to suppress the RM instability there may be a regime where magnetic fields can be used to redistribute vorticity through an interface and increase mixing. This effect is likely unique to the perpendicular orientation and the cylindrical interface.

While the magnetic fields drive the MHD effects, the mach number drives the hydrodynamics and exploring the initial Mach number in this work revealed another competing effect. Its known that for the RM instability, an increasing Mach number leads to increased interface mixing due to the increased baroclinic vorticity deposition. However, the opposite was observed for the MHD RM instability. Qualitatively, the higher Mach number cases further compressed the interface in the MHD RM 
instability. However, this actively drove down mixedness by compressing the diffusion layer, the area where mixing could occur. While the stronger deposited vorticity worked to compress the interface it was advected away by higher Alfven velocities, due to the higher post-shock density. Despite this, it was also shown that the stronger vorticity deposition resulted in a more correlated but unmixed interface. Thus the higher Mach number cases exhibited less mixedness and higher vortex advection in MHD.

This work has shown that interface mixing is a function of the amount of vorticity deposition, the rate at which it is advected, and the direction it is advected by Alfven waves. This leads to a rich parameter space where some counterintuitive results are found in mixing measures. Further simulations are planned to explore these results further and study the MHD RM instability with extended MHD effects, resistivity, two-fluid modeling, and in 3D. The authors of this paper are also planning to investigate the parameters shown within this work through experiments planned at the University of Missouri Fluid Mixing and Shock Tube Laboratory. 


\section{Chapter 4}

\section{D Simulations of the Cylindrical Magnetohydrodynamic Richtmyer-Meskhov Instability}

This chapter presents 3D simulations on a cylindrical Richtmyer-Meshkov Instability in Magnetohydrodynamics. Here a study of the effects of the orientation magnetic field relative to the shock wave transit direction is presented in an effort to quantify and qualify the evolution of the cylindrical MHD RM instability. The orientations considered herein are either parallel or perpendicular to the shock wave motion and are considered with a magnetic field strength of 500 gauss $(\mathrm{G})$. The driving Mach number is initialized at $M=1.66$. These parameters were selected to be easily achievable in experiments while the interface perturbation was selected such that its evolution is independent of either the shock wave or magnetic field orientations independently. It was found that the MHD RM instability evolution is dependent upon the magnetic field orientation relative to the shock transit direction. This is because the mechanism of suppression, attributed to Alfven waves, is a function of the magnetic field strength and the orientation of the magnetic field. This chapter 
expands upon the previous chapter by adapting the $2 \mathrm{D}$ cylinder into $3 \mathrm{D}$. This is motivated by the future experiments, which will be on an atmospheric plasma cylinder and may exhibit some 3D effects.

\subsection{Computational Enviroment: Ares}

The 3D simulations presented in this chapter were performed using the hydrocode Ares. Ares is a multi-material multi-physics hydrodynamics code developed at Lawrence Livermore National Laboratory (LLNL). Ares allows for Arbitrary Lagrangian Eulerian (ALE) calculations. This method utilizes a deformable Eulerian grid, similar to a Lagrangian scheme, which is then remapped back to the Eulerian grid [108]. This allows for superior interface tracking and momentum calculations when compared to purely Eulerian computations, and improved stability and efficiency over purely Lagrangian calculations [109]. Ares is fully second order in space and utilizes a second-order predictor-corrector in time [110]. This code also utilizes a staggered grid, so that the velocities are defined at the mesh nodes while fluid properties, such as density, are stored at the zone centers.

For increased efficiency and reduced computational time, Ares utlilizes Adaptive Mesh Refinement (AMR). This allows the user to refine/derefine the mesh about some local gradient to increase nodes in areas of greater interest and decrease nodes where the physics are of lesser interest. This mesh refinement is applied during the remapping from the Lagrangian time step to the Eulerian grid. The simulations in this chapter refine the mesh about the shock wave to prevent additional entropy waves from being generated due to the numeric scheme as well as around the interface to capture hydrodynamic growth, accoustic effects, and MHD waves. The areas upsteam and downstream of the interface and shock wave were held at the lowest 


\begin{tabular}{c|c} 
Table 4.1: 3D Simulation Domain and Mesh \\
\hline$X(\mathrm{~cm})$ & $0<X<300$ \\
$Y(\mathrm{~cm})$ & $-10<Y<10$ \\
$Z(\mathrm{~cm})$ & $0<Z<5$ \\
AMR Levels & 5 \\
$\mathrm{dx}($ Level=0) $(\mathrm{cm})$ & 1.33 \\
$\mathrm{dx}($ Level=5) $(\mu \mathrm{m})$ & 165
\end{tabular}

level of refinement. The lowest refined mesh size is $1.333 \mathrm{~cm}$ and at the highest level is approximately $165 \mu \mathrm{m}$ in $X$. Table 4.1 below shows the domain size, max levels of refinement, and mesh size at levels 0 and 5 . The interface, held at the highest resolution, is $2 \mathrm{~cm}$ in diameter and $5 \mathrm{~cm}$ in height. Therefore there are approximately 98 nodes per diameter (X-Y) at each Z and approximately 340 nodes in X-Z. At first glance this may seem like a moderate resolution, but since these simulations were run in 3D with AMR they took approximately 21,600 cpu hours to run without MHD this resolution was deemed acceptable.

Ares utilizes the ideal MHD equations, shown in the Introduction as Eqs. 1.17 through 1.15 and utilizes a Poisson solver for divergence control. In addition to treating the materials as perfect conductors, in this work Ares treats all fluids as ideal gases, gamma law equation of state shown as Eq. 1.9. While Ares has resistivity capabilities, this work does not consider two fluid MHD, resistivity, or Hall effects. Ares also can implement viscosity, diffusivity, and subgrid scale turbulence models though none of these models were turned on for this work.

\subsubsection{Simulation Parameters and Initial Conditions}

The simulation parameters for all cases are given in Table 4.2. All simulations consider a cylindrical interface of perfectly conducting nitrogen gas initialized at $T_{p}=2500$ 
Table 4.2: Initial Conditions for 3D Cylindrical Simulations

\begin{tabular}{|c|c|c|c|}
\hline & $B$ & $B_{\|}$ & $B_{\perp}$ \\
\hline$B(\mathrm{G})$ & 0 & 500 & 500 \\
\hline$\beta$ & - & 100 & 100 \\
\hline$P_{0}(\mathrm{~Pa})$ & & 101,325 & \\
\hline$T_{1}(\mathrm{~K})$ & & 300 & \\
\hline$T_{2}(\mathrm{~K})$ & & 2500 & \\
\hline$\rho_{\text {air }}\left(\mathrm{kgm}^{-3}\right)$ & & 1.12 & \\
\hline$\rho_{N_{2}}\left(\mathrm{kgm}^{-3}\right)$ & & 0.136 & \\
\hline$A$ & & 0.79 & \\
\hline M & & 1.66 & \\
\hline$D_{i}(\mathrm{~cm})$ & & 2 & \\
\hline
\end{tabular}

$\mathrm{K}$, with a diameter of $2 \mathrm{~cm}$ and a height of $5 \mathrm{~cm}$. The interface bulk temperature was selected in regards to the process of ionizing nitrogen at atmospheric conditions. The interface is surrounded by air at $300 \mathrm{~K}$, the unshocked region is initialized at standard atmospheric pressure.

Figure 4.1 shows the initial conditions common to all 3D simulations. Figure 4.1 A) shows contours of the interface and the shock wave. Here the interface cylinder is annotated for size and represented by a red contour. The shock wave is represented by a black planar contour in $\mathrm{Y}-\mathrm{Z}$ and annotated to show it travels in $\mathrm{X}$. On the left, Figures 4.1 B) and C) show mid-plane annotated pseudo-colors of plasma mass fraction (species 2 per the Atwood number definition) given as $\Upsilon_{P}$ in $\mathrm{X}-\mathrm{Y}$ and $\mathrm{X}-\mathrm{Z}$ respectively. Here the shock wave is represented by a planar black line. A diffuse interface was chosen to due to the large temperature difference between the interface and its surroundings.

These slices show the interface at time $t=0$ and are centered around the interface, this scheme will be used repeatedly throughout this work. Future slices will focus on a $6 \mathrm{~cm}$ by $6 \mathrm{~cm}$ area centered on the interface, while this will neglect to show the boundaries of the simulation it will allow for better viewing on the evolution of the 

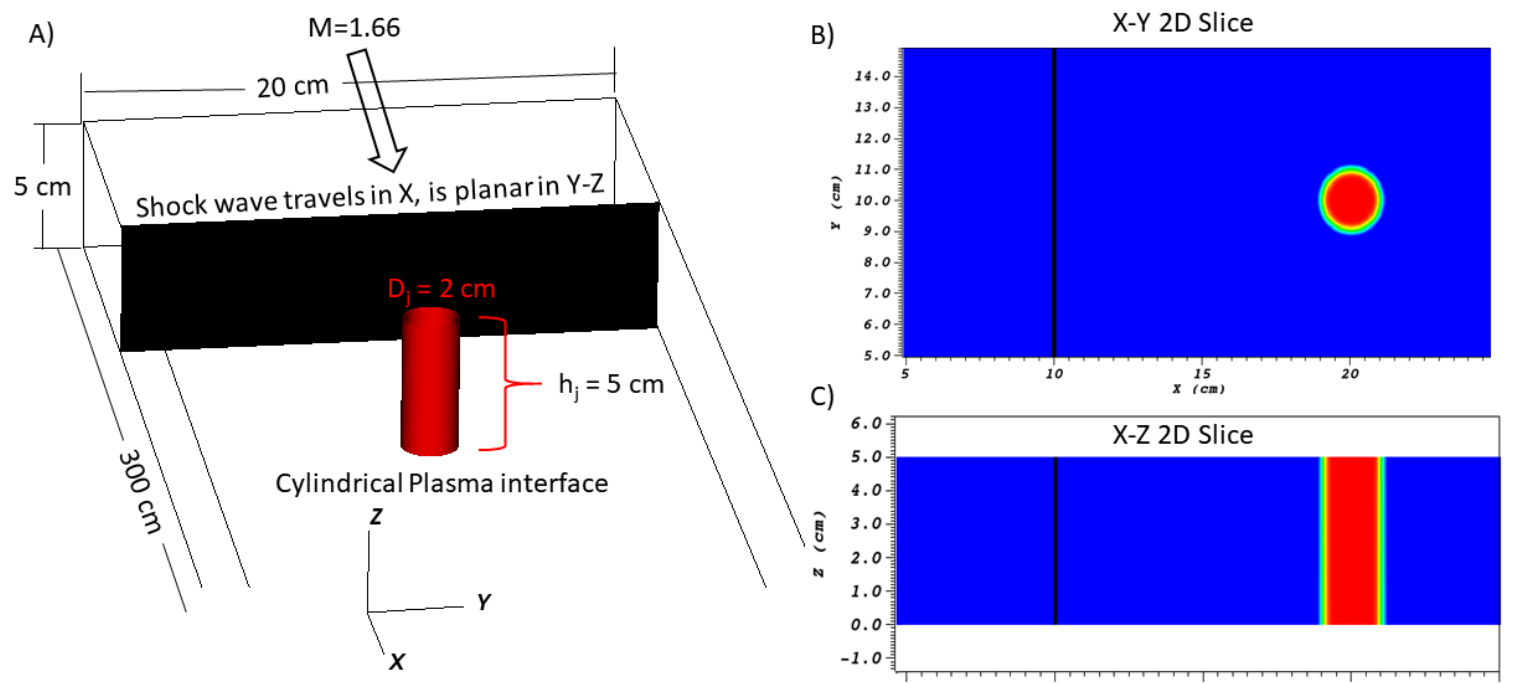

C)

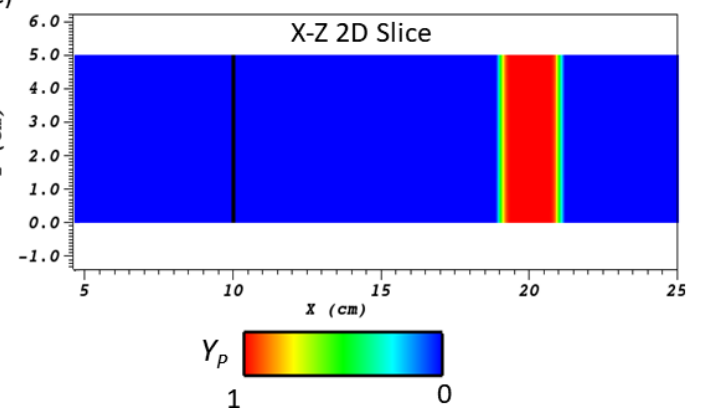

Figure 4.1: Initial conditions for all 3D simulations presented in Chapter 4. A) Contour plot with the cylindrical interface (red) and the shock wave (black) annotated for size and magnitude, here it can be seen that the shock wave travels in X B) X-Y 2D pseudo-color slice for species C) X-Z 2D pseudo-color slice for species, in both of $\mathrm{B})$ and $\mathrm{C}$ ) the shock wave is represented by a black line 
RM instability and the MHD effects. The full domain is $300 \mathrm{~cm}$ by $200 \mathrm{~cm}$ by $5 \mathrm{~cm}$ in $\mathrm{X}-\mathrm{Y}-\mathrm{Z}$ with reflecting boundary conditions at all boundaries. This means that all $\mathrm{X}-\mathrm{Y}$ midplane slices shown below are sliced at $Z=2.5 \mathrm{~cm}$. All images are visualized using the computational post-processing software VisIt. VisIt is supported by the Department of Energy with funding from the Advanced Simulation and Computing Program and the Scientific Discovery through Advanced Computing Program[111].

To investigate the effects of the magnetic field on the MHD RM instability three simulations are presented. The traditional RM instability, $B=0$ is compared to cases with a perpendicular, $B=B_{\perp}$, or parallel magnetic field, $B=B_{\|}$, of 500 Gauss $(\mathrm{G})$. These cases all share $M=1.66$, a Mach number realizable within the Missouri Shock Tube [84], and $A=0.79$. The magnetic field strength of $500 \mathrm{G}$ was selected as it has shown significant suppression in the shock-bubble problem in MHD [3] and is relatively easy to achieve within experiments. For simplicity the cases will be referred to by the magnetic field orientation. Hence, the traditional RM will be called $B 0$ while the magnetic field in $\mathrm{X}$ and $\mathrm{Y}$ will be $B_{\|}$and $B_{\perp}$ respectively.

\subsection{Results}

For a traditional cylindrical RM instability with these initial conditions, $M 1.7 B 0$, significant RM instability development occurs as early as $t=0.5 \mathrm{~ms}$ post shock. As such the following results will be taken out to $t_{p s}=500 \mu \mathrm{s}$. where the subscript $p s$ represents post shock. The evolution of all three simulations are shown below in Fig. 4.2. Here, contours of the interface are shown at $t_{p s}=100,250$, and $500 \mu \mathrm{s}$. The first column shows both the mid-plane species pseudo-color and the evolution of the contour for the traditional RM instability. At early time a small bubble front can be seen while two large roll-up structures form and store the majority of the species mass 
fraction. This bubble front is below the minimum threshold for the contour, which was selected to be greater than $25 \%$ mass fraction. These roll up structures continue to mix as the bubble front vanishes by mid-time. Here the interface contour appears as two circular objects. At late time the contour is mostly round and appears to be shrinking in diameter. This can be seen to be due to mixing in the pseudo-color as the species is being further mixed.

The magnetic cases exhibit additional behavior in the contour plots. The species mass fraction pseudo-colors are not presented in this figure, however considering the RM pseudo-color the contours can give good insight into the evolution of these MHD RM instabilities. In addition to visualizing the interface, Alfven waves are also shown as semi-transparent black contours. It was mentioned previously that the mechanism of suppression is the Alfven waves, which propogate along the magnetic field lines and work to remove vorticity from the interface. These contours represent a small fraction of the actual Alfven waves and are shown here to indicate their effects and growth - they are not meant to show the effects in totality.

The $B_{\|}$case shows a thick bubble front at early time, already deviating from the RM case. Alfven waves can be seen around the interface perimeter. These waves have grown by mid-time moving away and through the interface. They are significantly stretched in the direction of shock transit, or along the parallel magnetic field. At this time, the interface has formed roll-up features, having mixed the bubble front. At late time the roll-ups have been considerably stretched when compared to the RM interface.

Like $B_{\|}$, the $B_{\perp}$ case shows a bubble front at early time. Unlike the other two cases however, there appears to be no notable rollup features at this time. At midtime the interface shows suppression and maintains a thick bubble morphology, more similar to a compressed circle than a RM instability. Here the Alfven waves seem 


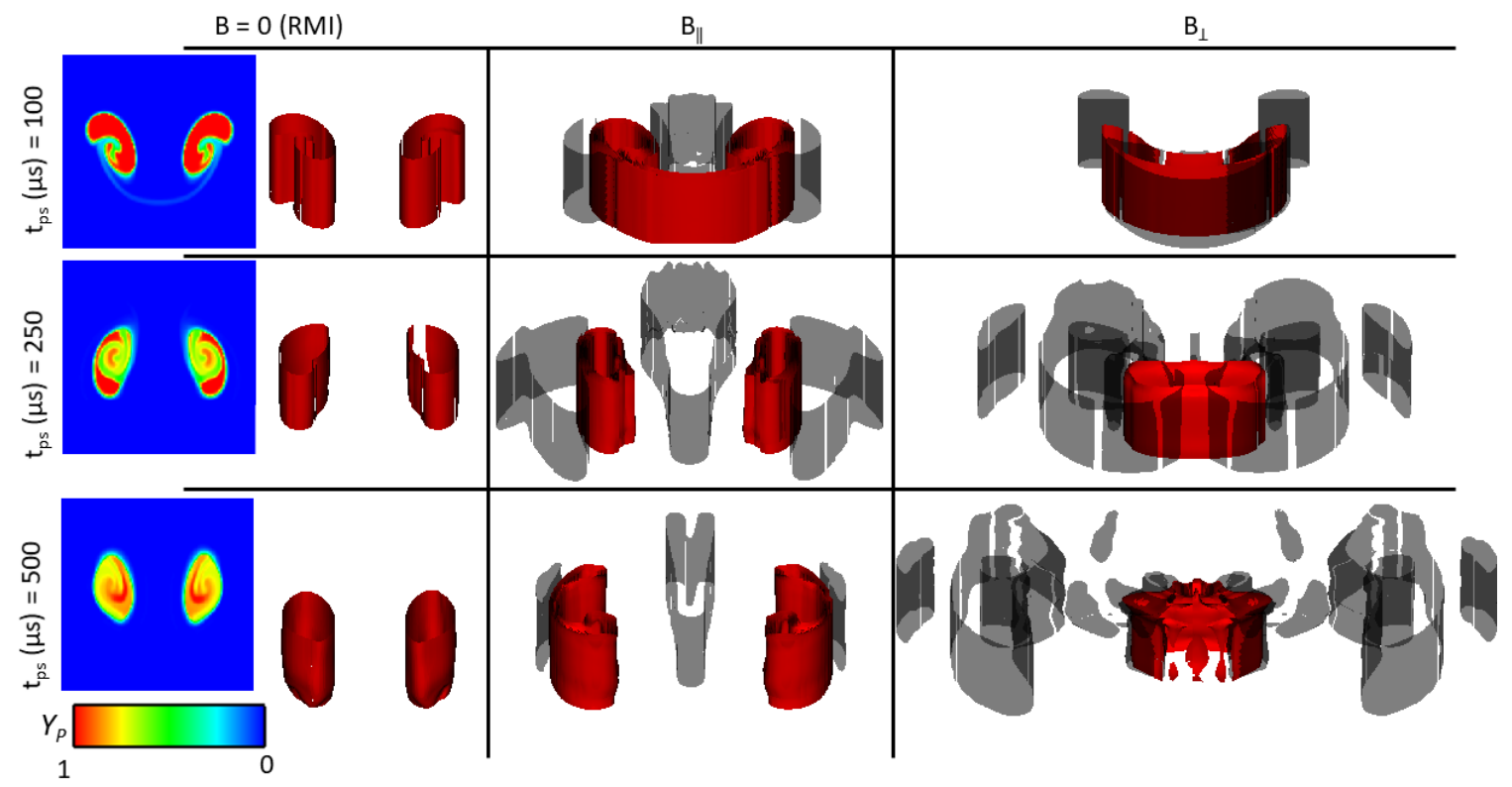

Figure 4.2: Contour plots for the interfacial evolution of $B 0, B_{\|}$, and $B_{\perp}$ cases, where a red surface is meant to represent the $5 \%$ species contour. The mid-plane species pseudo-color is included for $B 0$ as reference. Both the magnetic field cases show representative contours for Alfven waves, shown as semi-transparent black surfaces

to be moving outward laterally from the interface, again mostly following the now perpendicular magnetic field. At late time the interface contour has inverted, not yet forming roll-ups or exhibiting notable RM growth.

It was mentioned in the introduction that the RM is responsible for undesirable mixing in HED applications. This can be viewed, not only as the mixing of one species into its surrounding, but also the breakdown of the temperature gradient. As such, the mid-plane temperature pseudo-colors are shown for each case in Fig. 4.3. This variable was selected as it closely follows the species behavior (see the species pseudo-color for $B 0$ in figures 4.2 and 4.3) but also because it is desirable in some applications to reduce the amount of mixing to preserve high temperatures. Here the early discussion for the contours can be clearly seen again. $B 0$ exhibits the formation of roll-ups at early time which work to break down the temperature 
(and therefore species) through mixing with the surrounding air. By mid-time the bubble front is mixed, and by late time the majority of the hot gas is mixed and lowered in temperature. $B_{\|}$shows similar results but maintains a small bubble front at $t_{p s}=250 \mu \mathrm{s}$. At this time the roll-up features dominate the growth, but do not appear to actually be mixing. By late-time these features are significantly stretched in the direction of the magnetic field, but still maintain two hot spots.

$B_{\perp}$ shows the most significant deviation from the other cases. At early time the thick bubble front is evident, and no roll-up features can be seen. At mid time the interface has become thicker, but the majority of the hot gas is still unmixed. No roll up features are evident. By late time the interface has inverted itself. Considering the Alfven contours shown in Fig. 4.2, it's likely this is because the Alfven waves have traveled through the interface along the magnetic field lines carrying vorticity of alternating signs and inverting the interface. However, despite its inversion there is significantly less qualitative mixing in this case than in the other cases.

The mechanism of mixing in the RM instability is the vorticity deposited by the interaction between the misaligned pressure and density gradients. To understand how the vorticity, Eq. 4.1, is affected by MHD effects, pseudo-colors at various heights in Z have been plotted below in figures 4.4-4.6. Figure 4.4 shows the vorticity fields for B0. There appears to be little 3D effects as the vorticity fields are relatively similar at all time across each height. This follows logically from the contour plots shown earlier, which didn't appear to have any significant 3D formations. This is idealized in simulation space, as most experiments may have some small 3D perturbations which will grow during the instability. Aside from the uniformity in $\mathrm{Z}$, this figure also shows the expected RM vortex organization. The vortex cores are strongly positive/negative, and remain tight and relatively high magnitude through late time. These vortex cores work to break down the interface and mix with the surrounding 


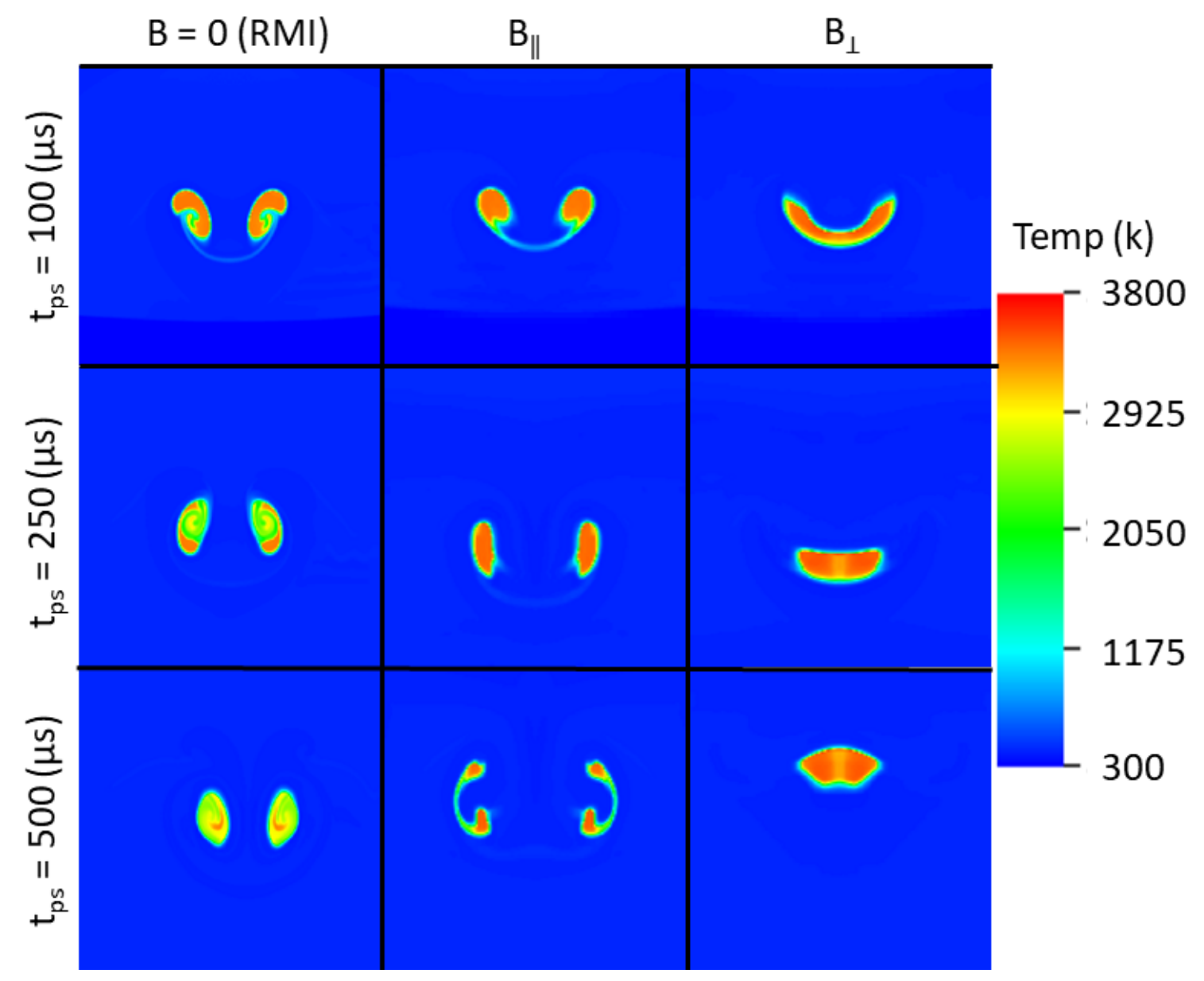

Figure 4.3: Mid-plane $(\mathrm{Z}=2.5 \mathrm{~cm})$ Pseudo-color for temperature with respect to post shock time 


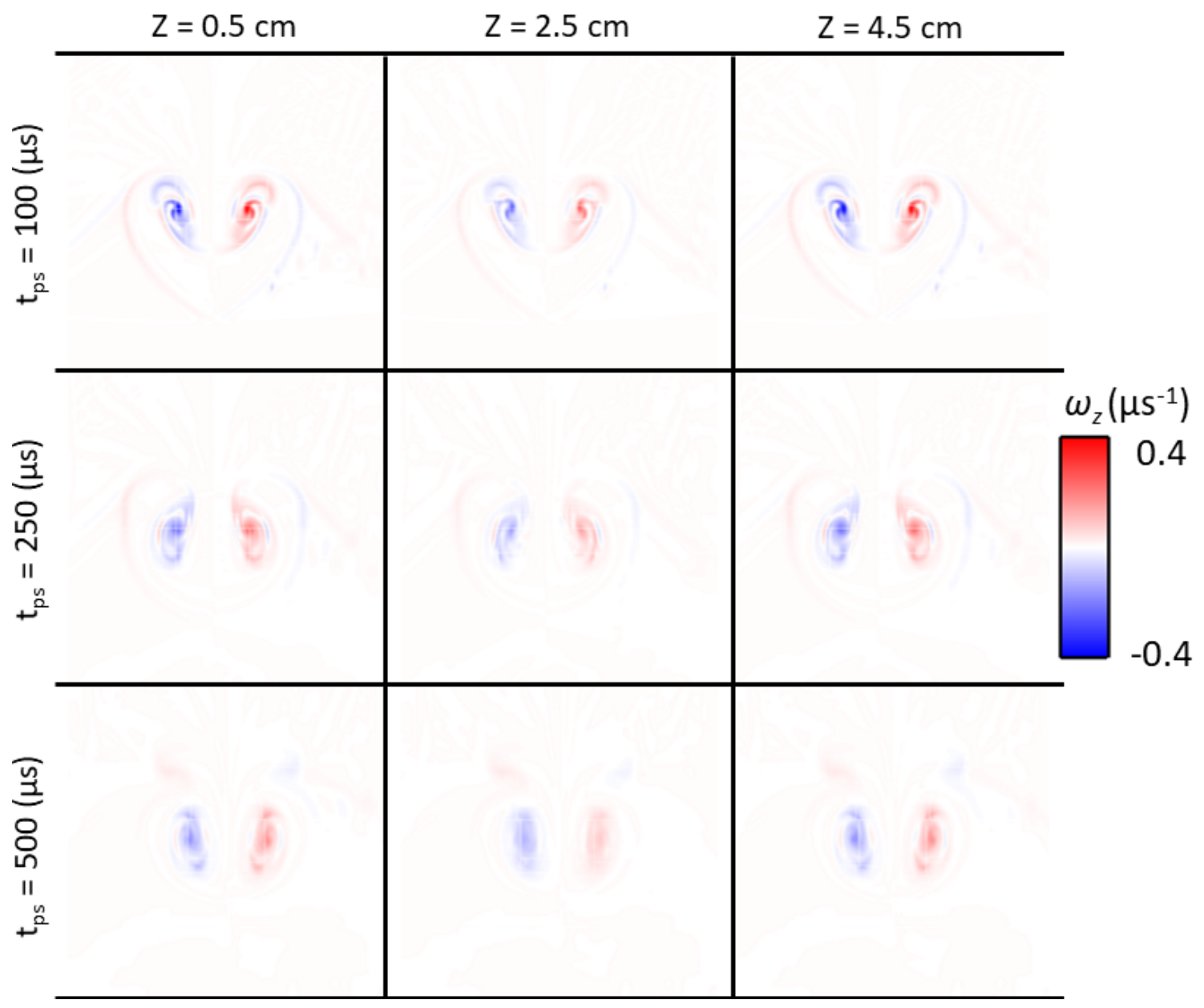

Figure 4.4: 2D vorticity pseudo-colors at various $\mathrm{Z}$ planes for $B 0$

air.

$$
\vec{\omega}=\nabla \times \vec{u}
$$

Similar to the vorticity field in Fig. 4.4 the vorticity field for $B_{\|}$, Fig. 4.5 is relatively consistent in $\mathrm{Z}$. It also has very similar structure to the traditional RM instability through time. However, even at early time some differences can be seen. $B 0$ has strong central cores visible at $t_{p s}=100 \mu \mathrm{s}$, however at this time $B_{\|}$already exhibits a stretched vortex core with little to no visible central vorticity. By mid-time 


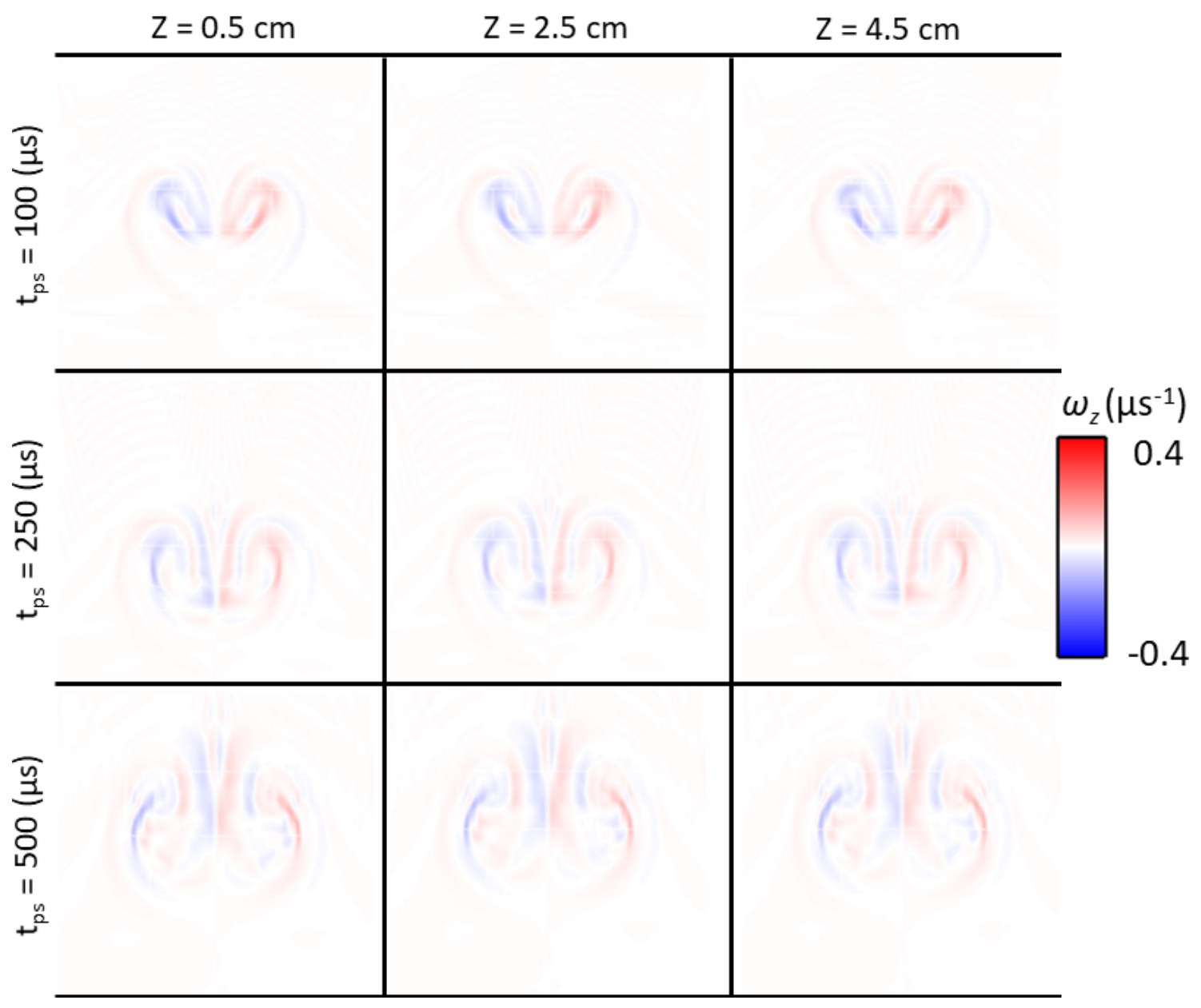

Figure 4.5: 2D vorticity pseudo-colors at various $\mathrm{Z}$ planes for $B_{\|}$

the vorticity field has been stretched until late time some interaction has occured between the positive and negative vortex tails. These tails, and their interactions, form due to the Alfven stretching of the vorticity field. However, since the majority of the Alfven effects are parallel to the shock transit direction the general organization of the vorticity is the same as $B 0$.

As can be expected from the previous figures, $B_{\perp}$ exhibits significant evolution differences from either of the previous cases. Even at early time no vortex cores have formed, instead strong alternating vorticity sheets dominate the vorticity field. As these sheets are stretched along the magnetic field lines, their magnitudes rapidly 
decrease. This is because the interaction of equal strength alternating vorticity sheets works to drive the field to zero vorticity. It can also be seen that unlike the previous two cases, the dominant vorticity has been flipped. That is to say, what would be a vortex core on the left side (at early time) is the opposite sign for this case when compared to the previous two cases. This is likely due to the Alfven waves moving the vorticity through the interface and stretching the field perpendicularly relative to the shock transit direction. The interaction between the alternating signs and how the field is stretched due to MHD effects is also responsible for the inversion of the interface at late time (see contour and temperature pseudo-color in figures 4.2 and 4.3 respectively).

Enstrophy, Eq. 4.2, is used to quantify the energy in the vorticity. Presented in Fig. 4.7, enstrophy is integrated across the entire simulation domain to capture the vortical energy in the $\mathrm{X}-\mathrm{Y}, \mathrm{X}-\mathrm{Z}$, and $\mathrm{Y}-\mathrm{Z}$ planes. This figure represents the enstrophy for $B 0, B_{\|}$, and $B_{\perp}$ with black, red, and blue lines respectively. Calculating the total enstrophy across the total domain also serves to capture any vorticity that might have been removed from the interface due to the MHD waves. Since the MHD effects are dependent upon the vorticity, or any perturbation across the density interface across the magnetic field lines (Eq. 1.14), it is expected that the baroclinic vorticity is the same between all three simulations. Indeed this initial deposition can be seen to be similar between the cases and occurs just before $t_{p s}=0$. However, after this $B 0$ shows a increase in enstrophy. This case exhibits greater enstrophy through time, which follows as its vortex fields show greater organization and greater magnitude.

$$
\epsilon=\int \vec{\omega} \vec{\omega} d A
$$

$B_{\perp}$ generally exhibits the lowest enstrophy though time, as the alternative vortic- 


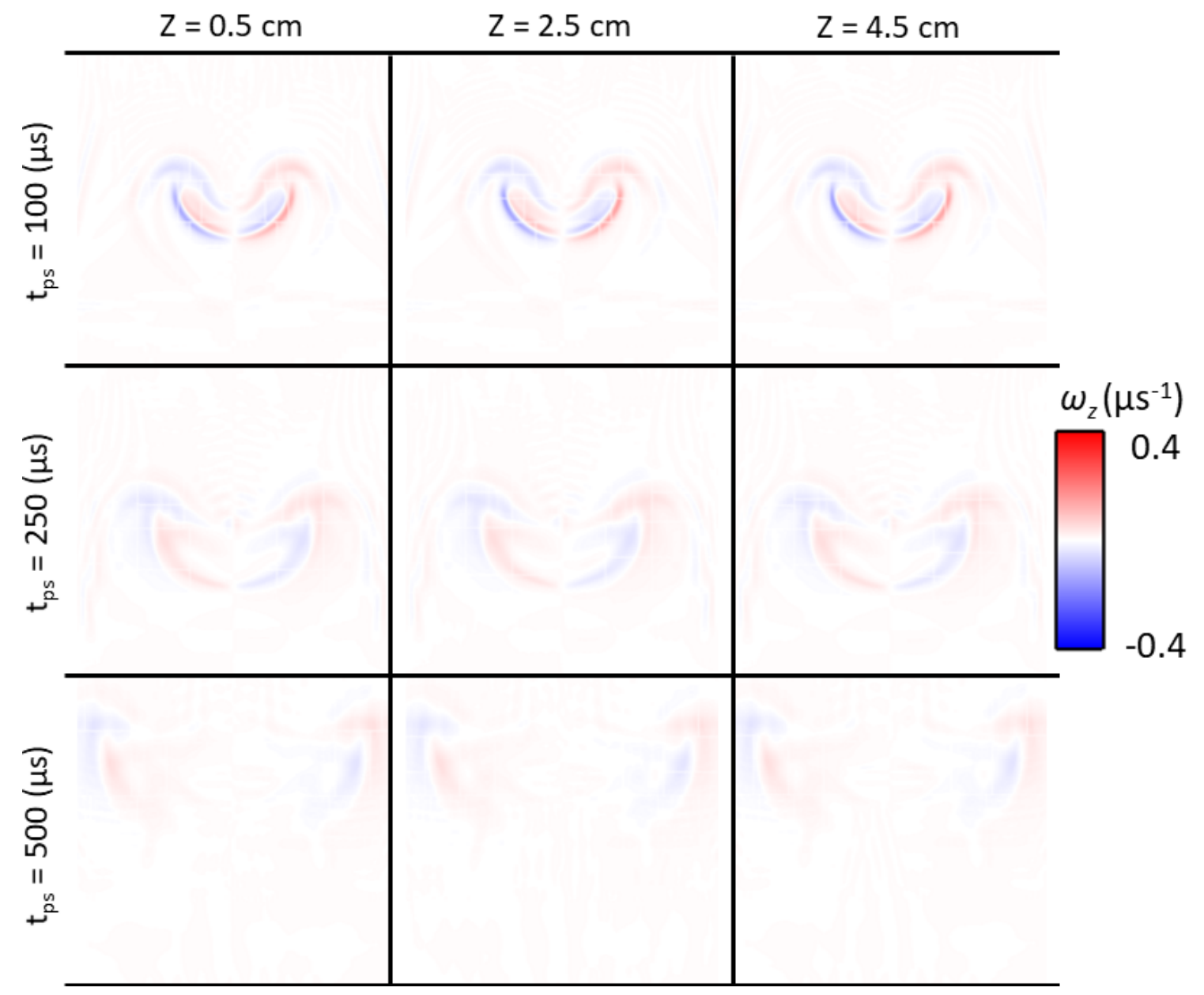

Figure 4.6: $2 \mathrm{D}$ vorticity pseudo-colors at various $\mathrm{Z}$ planes for $B_{\perp}$ 


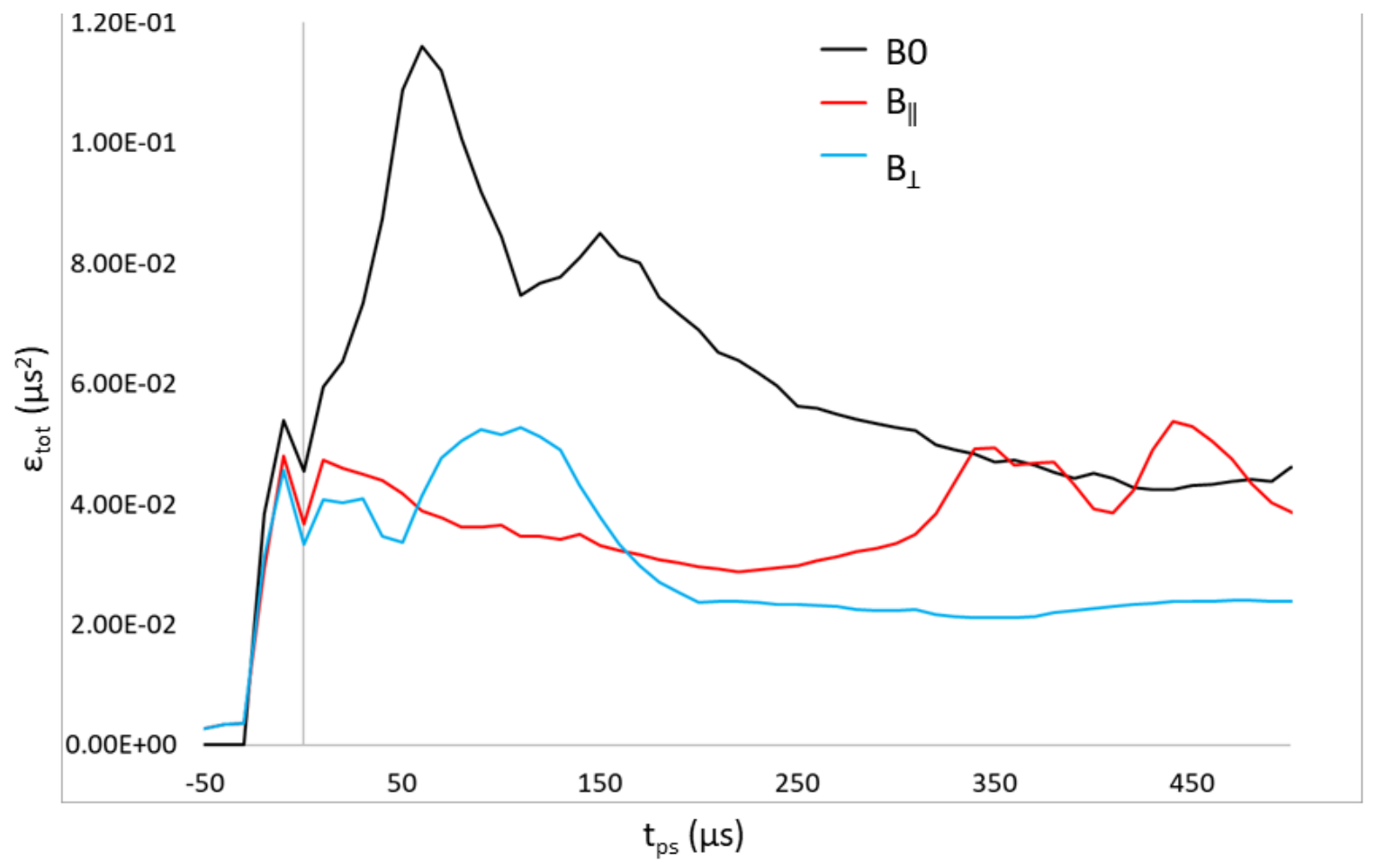

Figure 4.7: The total enstophy calculated across the entire domain for $B 0$ (Black), $B_{\|}\left(\right.$Red), and $B_{\perp}$ (Blue) 


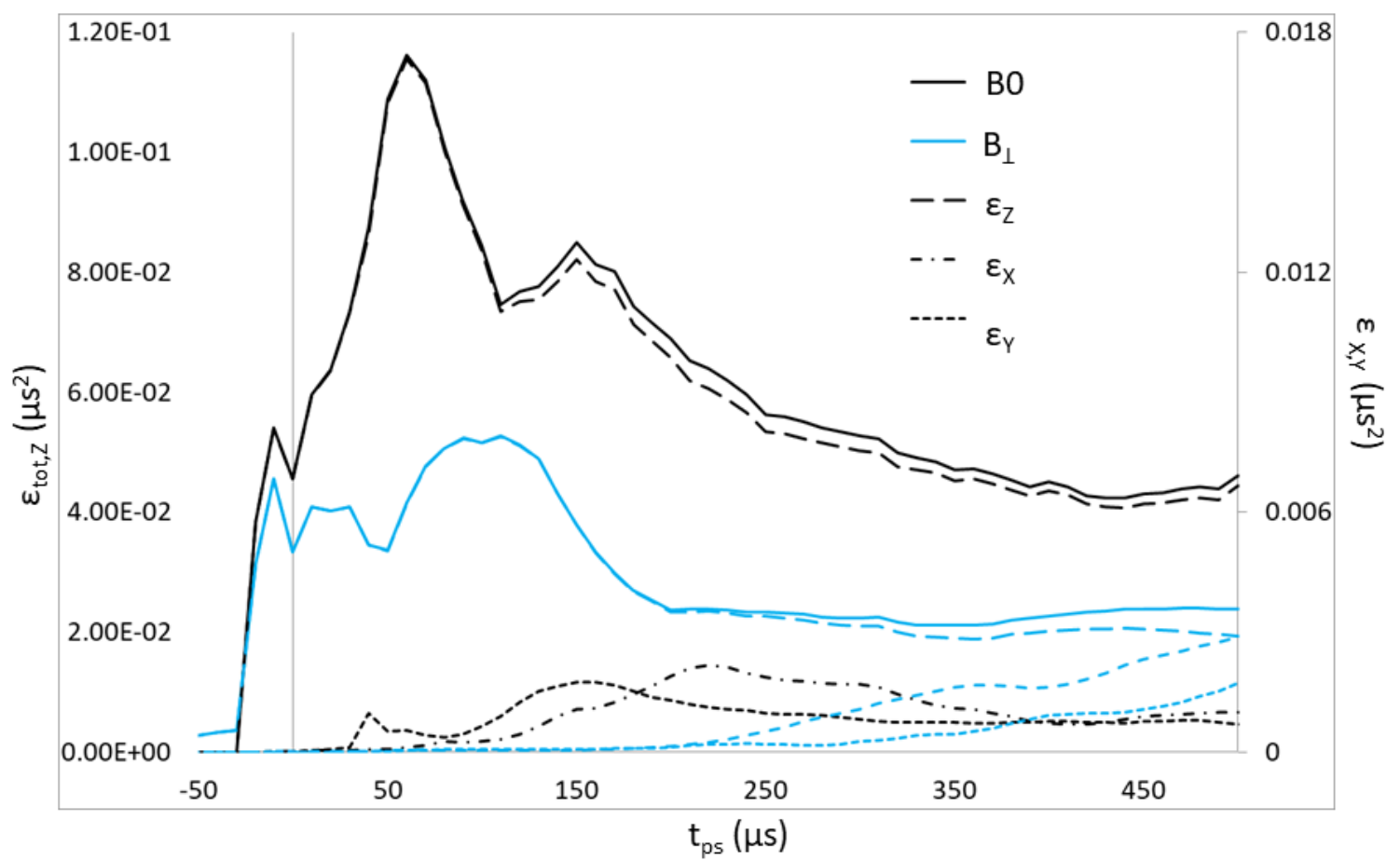

Figure 4.8: The total enstophy (solid), enstrophy in X (dash-dot), enstrophy in $\mathrm{Y}$ (small dash), and the enstrophy in $\mathrm{Z}$ (large dash) calculated across the entire domain for $B 0$ (Black) and $B_{\perp}$ (Blue)

ity sheets are forced to interact along the perpendicular field lines and the competition causes some vortex annihilation. There is a small enstrophy peak around $100 \mu \mathrm{s}$, similar to the peak in enstrophy for $B 0$ at $50 \mu \mathrm{s}$, this is due to the interaction of vorticity with the interface generating secondary baroclinic depositon. $B_{\|}$has a similar bump to $B 0$ at $t_{p s}=100$ and then again at $t_{p s} \approx 350 \mu \mathrm{s}$. The late increase in enstrophy is likely due to the vorticity responsible for the interface stretching that occurs to drive the interface from the morphology visible at $t_{p s}=250$ to $t_{p s}=500 \mu \mathrm{s}$.

While there appeared to be little variation in the interface growth in $\mathrm{Z}$ when comparing the contours and the pseudo-colors, it may be of interest to see what contributions the vorticity in $\mathrm{X}$ and $\mathrm{Y}$ have to the total enstrophy. As such, Fig. 4.8 shows the total enstrophy (solid line) as well as the $\mathrm{X}$ (dash-dot), Y (small dash), 
and the $\mathrm{Z}$ (large dash) contributions for $B 0$ and $B_{\perp}$. The left axis is the same as Fig 4.7, whereas the right axis represents the $\epsilon$ in the $\mathrm{X}$ and $\mathrm{Y}$ planes. For clarity, $B_{\|}$ was neglected, however it shows the same trends as $B_{\perp}$. As expected, the majority contribution to the total enstrophy is the Z-component. This makes sense as the majority of RM growth can be seen in the X-Y planes, meaning it's $\omega_{Z}$ driven. For $B 0$ the $\mathrm{X}$ and $\mathrm{Y}$ components maintain relatively little contribution to the total enstrophy, however $B_{\perp}$ exibits a late time increase $\epsilon_{X}$ and $\epsilon_{Y}$. While these increases may seem significant they are still three orders of magntiude below $\epsilon_{Z}$.

In effort to explain why the interface mixing has differed from the traditional RM instability in the MHD cases the vorticity and enstrophy have been studied. However, vortex advection and stretching as well as the secondary spikes in enstrophy can complicate the argument that the RM is suppressed quantitatively. In an effort to capture how the vorticity affects the species gradient, or causes mixing, a correlation coefficient was created to relate the species gradient and vorticity as shown in Eq. 4.3.

$$
C C=\frac{\left\langle\left|\nabla \Upsilon_{P}\right||\omega|\right\rangle}{\sqrt{\left\langle\left|\nabla \Upsilon_{P}\right|^{2}\right\rangle\left\langle|\omega|^{2}\right\rangle}}
$$

The correlation coefficient relating the species gradient and vorticity, referred to simply as the correlation coefficient, is plotted in Fig. 4.9. Without consideration this shows a slightly concerning story. At face value, it may appear that $B_{\|}$exhibits greater interaction between vorticity and the species gradient at late time than $B 0$. However, its more accurate to say that the vorticity in $B 0$ remains relatively constant and works to break down the species gradient driving a lower, but more consistent, correlation. At late times an increase in enstrophy is visible in $B_{\|}$, Fig. 4.7. This increase in enstrophy, and therefore vorticity, is a secondary interaction when the 
stretched field works on a significantly stronger species gradient, when compared to the gradient at this time in $B 0$. So while $B 0$ has superior vorticity and qualitative mixing, the species gradient is stronger in $B_{\|}$driving up the late time correlation. Similarly $B_{\perp}$ shows significant correlation early time, which rapidly decreases and remains lower than the other cases. This is again the interaction of the vorticity as it moves through the species gradient. So while $B_{\|}$is driven to greater correlation at late time, $B_{\perp}$ is driven to lower correlation at late time all due to the Alfven waves moving the vorticity along the field lines. This metric has been used as a metric of mixing [3], its potentially more accurate to say its a metric of when mixing is occuring. Since the correlation is steady throughout time, $B 0$ experiences more mixing throughout the simulations. $B_{\perp}$ experiences more mixing in early time, causing the half-moon like shape but never has its species gradient truly mixed with its surroundings hence the ever decreasing correlation. Meanwhile, $B_{\|}$experiences some roll-up formation and some early time mixing, and then again experiences late time mixing - streching the interface and forming two cores, Fig. 4.3.

\subsection{Conclusions}

This work studied the 3D cylindrical MHD RM instability by varying the magnetic field orientation relative to the shock transit direction. The cylindrical interface was selected as its evolution is independent of the orientation of either the shock wave or the magnetic field direction. Instead, the evolution depends only on the orientation of the shock transit direction relative to the magnetic field. It was shown that interface evolution is a function of the amount of enstrophy, the growth of the vorticity field, and the advection direction via Alfven waves. This leads to a rich parameter space where some counterintuitive results are found in mixing measures. To understand 


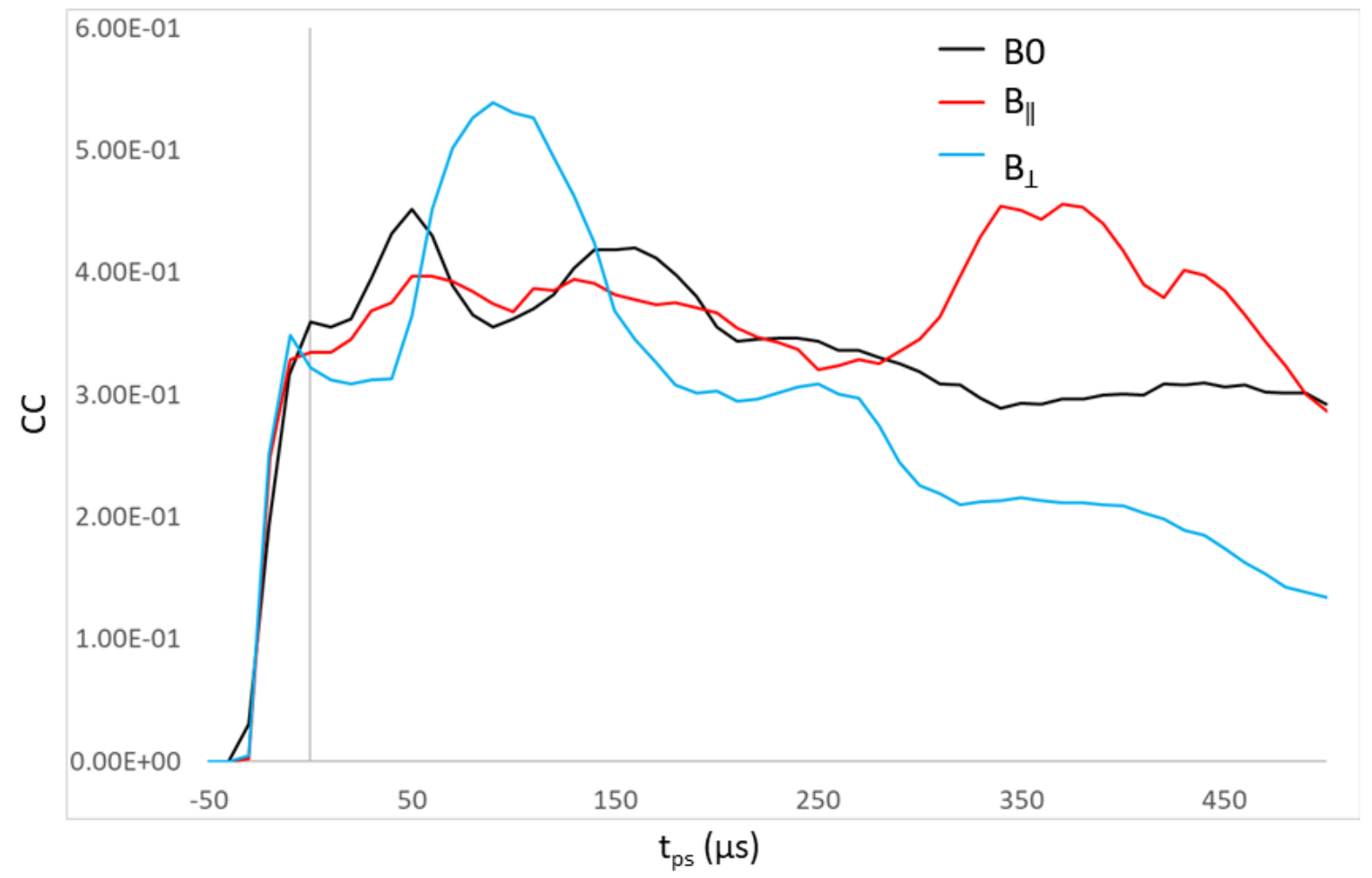

Figure 4.9: The correlation coefficient, indicative of when mixing occurs and the scales of mixing, for $B 0$ (Black), $B_{\|}$, and $B_{\perp}$ 
the parameter space the simulations were observed qualitatively via species contours, as well as temperature and vorticity fields and quantitatively through enstrophy and the correlation coefficient of the vorticity and species gradient. It was found that in general the presence of the magnetic field suppresses RM instability-like evolution by the advection of vorticity with a preferential field orientation resulting in greater suppression.

Expanding upon results found in the literature, it was shown here that the presence of either a perpendicular or parallel magnetic field in the MHD RM instability suppresses RM instability evolution; with a perpendicular magnetic field showing less correlation and significantly reduced RM instability morphology compared its parallel field counterpart. This is because in the presence of a magnetic field the whole of the deposited baroclinic vorticity, which drives the evolution in the RM instability, no longer remains on the density gradient. The differences in field orientation are further highlighted by the vorticity field and enstrophy. The vorticity is stretched significantly in the direction of the magnetic field, hence the parallel field stretches the vorticity along the shock wave direction. Enstrophy versus time curves, for total enstrophy and the enstrophy components show that the MHD cases result in lower total enstrophy. Furthermore, vorticity is able to traverse the interface rapidly in the perpendicular field orientation, allowing positive and negative vorticity from opposite sides of the interface to interact and compete, diminishing the enstrophy.

\subsubsection{Future Resistivity Work}

While this 3D study is informative, the simulations here are considered as ideal. To better serve experimentalists it may be beneficial to utilize the resistive capabilities of Ares. Unfortuately, as of now these simulations are computationally prohibitive. 
To understand if resistivity is necessary, the relevent physics question is "What is the magnetic diffusion time relative to the accoustic time scale for the perturbation?" [112] If the diffusion time is longer then resistivity can be ignored and ideal MHD will accurately capture the evolution. If the diffusion time is much shorter however, the magnetic field will diffuse quickly and the resistive simulations will trend towards the traditional RM. It's in between these extremes, where the accoustic and magnetohydrodynamic time scales are comparable, that the resistive calculations may yield interesting results. If one calculates the magnetic field diffusion coefficient, $D$ as Eq. 4.4 , where $\mu$ is the permeability and $\sigma$ is the plasma conductivity, then one can estimate the magnetic diffusion time scale by dividing a pertubration length scale by $D$, which has units of $m^{2} s^{-1}$. For the cylinder, this length scale is the area. To calculate this the conductivity of a plasma must be estimated, Eq. 4.5. The conductivity is a function of charged species number density, elementary charge, electron mass, and the collisional frequency or $n, e, m_{e}$, and $\nu$ respectively. Using the data from Fig. 2.12 , one cast estimate the degree of ionization to be $2.5 \%$, the diameter to be $\approx 4$ $\mathrm{cm}$, and the height of the ionized region to be $10 \mathrm{~cm}$. This gives $n$ to be $\approx 1.69 e 20$. The last component necessary to solve for the electrical conductivity is the collisional frequency. This can be estimated a number of ways, and is usually calculated from some known number density or conductivity. However, to illistrate the effects of ionization on diffusion time, Eq. 4.6 was used to calculate the electron-electron collision frequency. This is a function of the temperature in electron volts and $\Lambda$, which is known as the coloumb algorithm and is often taken to be a constant value of 10 [51].

$$
D=(\mu \sigma)^{-1}
$$




$$
\begin{gathered}
\sigma=\frac{n e^{2}}{m_{e} \nu} \\
\nu_{e-e}=5 \times 10^{-6} \frac{n \ln (\Lambda)}{T_{e V}^{3 / 2}}
\end{gathered}
$$

From these equations, one can estimate a diffusion time. Figure 4.10 shows the diffusion time with respect to the degree of ionization with curves of different magnitudes of collisional frequency, including the $\nu_{e-e}$. Usually, the collisional frequency is dependent upon the system, however here a range was considered to illustrate the regimes where different physics may be useful. The solid and dashed black lines show the RM evolution and the shock transit time for an interface of this size at $M=1.66$. Here it can be seen that as the collisional frequency decreases for this estimated conductivity the magnetic diffusion time scale grows far beyond the accoustic time scales for all ionization and therefore the simulations can be considered as ideal. However, for frequencies $\nu=1 e 6$ and greater the classic hydrodynamics simulations will capture the effects as the diffusion time is much less then the accoustic time scales. While this is just an estimated frequency range and ionization range, this tool can be helpful for future researchers in determining if resistive effects are necessary in simulating the MHD RM instability.

\subsection{Acknowledgements}

The work presented in this chapter was performed under the auspices of the U.S. Department of Energy by Lawrence Livermore National Laboratory under Contract DE-AC52-07NA27344. This document was prepared as an account of work sponsored by an agency of the United States government. Neither the United States government 


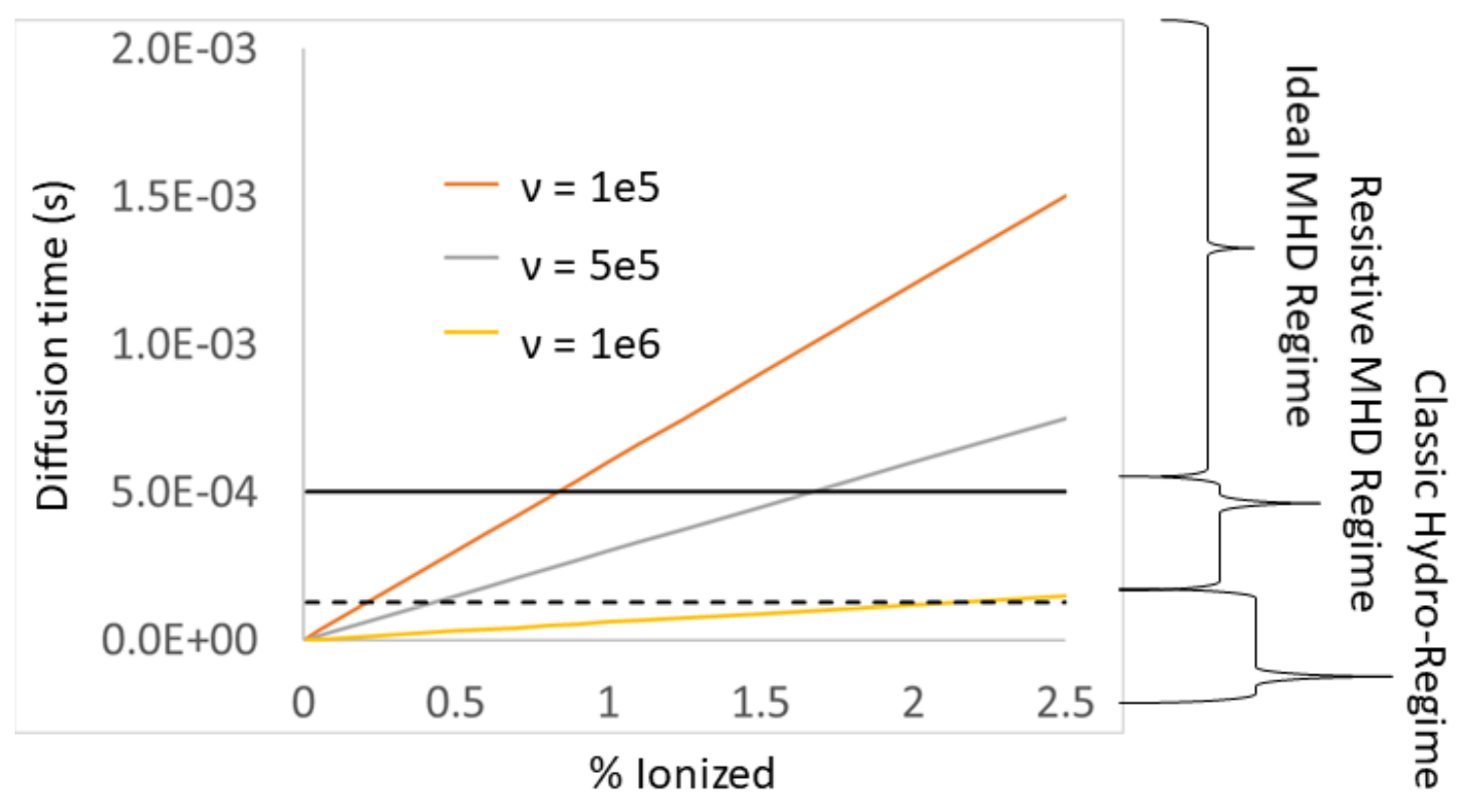

Figure 4.10: The magnetic diffusion time with respect to degree of ionization for various collisional frequencies

nor Lawrence Livermore National Security, LLC, nor any of their employees makes any warranty, expressed or implied, or assumes any legal liability or responsibility for the accuracy, completeness, or usefulness of any information, apparatus, product, or process disclosed, or represents that its use would not infringe privately owned rights. Reference herein to any specific commercial product, process, or service by trade name, trademark, manufacturer, or otherwise does not necessarily constitute or imply its endorsement, recommendation, or favoring by the United States government or Lawrence Livermore National Security, LLC. The views and opinions of authors expressed herein do not necessarily state or reflect those of the United States government or Lawrence Livermore National Security, LLC, and shall not be used for advertising or product endorsement purposes. LLNL-TH-772091. 


\section{Chapter 5}

\section{Summary and concluding remarks}

This document details research performed towards the completion of a Doctoral degree in Mechanical Engineering. The work presented here is divided into four chapters which cover background, experimentation, and simulation efforts towards understanding magnetohydrodynamic effects on shock driven instabilities, with experimental and 3D simulation focus on the Richtmyer-Meshkov (RM) Instability. Interest in the RM instability stems from a decades old desire to understand and support fusion experiments. The experiments and simulations assume a low energy university laboratory setting. However, the physics considered have strong applications towards the high energy density systems often found in astrophysics and national laboratory experiments. These physics include the generation of plasma, diagnostics for plasma characterization, shock driven hydrodynamics, multispecies/multiphase mixing, the presence of a magnetic field, and magnetic tension. A literature review for these topics encompassing previous theoretical, experimental, and simulation work is included in this introduction chapter. In Chapter 2 the developement and diagnosis of an atmospheric plasma torch is presented, and is closely based off of a publica- 
tion the author was closely involved in [2]. This sets up an interface to be used in future magnetohydrodynamic experiments to be done by the next generation of masters and doctoral students at the University of Missouri Fluid Mixing and Shock Tube Laboratory (MU-FMSTL). Chapters 3 and 4 detail simulation work performed using highly parallelized multiphysics hydrodynamics codes. First, Chapter 3 shows 2D simulations of an ideal magnetohydrodynamic shock-bubble problem has been published in Physical Review Fluids [3]. Chapter 4 presents 3D simulations of the Magnetohydrodynamic Richtmyer-Meshkov (MHD-RM) Instability. While this work consideres similar physics as Chapter 3 different numerical methods were explored and the calculations were taken into 3D in preparation to incorporate resistive effects for future work. The follow sections briefly discuss the conclusions of each chapter.

\subsection{The developement and characterization of an atmospheric non-transferred plasma source for magnetohydrodynamic experiments}

The development and diagnosis of a non-transferred DC arc atmospheric pressure plasma torch is presented in Chapter 2. A long laminar torch was developed and gas flow rates, arc currents, and arc voltages were explored. It was found that the jet length and total power are functions of axial flow rate. It was also found that the total jet length can be divided into two regions, a plasma region and an incandescent region. As the axial flow rate is increased the plasma region grows as the total jet length decreases until the torch transitions to turbulence and both decrease in size. Tangential flow was also explored and found to have a minimal effect.

Diagnosis of the plasma was performed utilizing a single langmuir probe, 2DMIM, and a particle Mie scattering method (particle image velocimetry). While the Lang- 
muir Probe measurements failed, the experiment as a whole seemed promising. It is recommended that the equipment be upgraded so that the plasma parameters can be found. It may also be beneficial to persue a double langmuir probe configuration, so that the biased and measure voltages are based on the circuit and may provide more stable readings. Despite the langmuir probes failure, the 2DMIM was able to provide electron temperatures and charge density. This data can be used for future experiments and simulations. Similarly, the PIV-like Me scattering images can be used to capture the interface in $R-Z$ and $R-\theta$ and are ready for use. Once MHD-RM instability experiments are run this method can be converted to full PIV and provide data to validate simulations.

\subsection{D Simulations of the Magnetohydrodynamic Effects in a Shock-Accelerated Gas Cylinder}

This work studied the cylindrical MHD RMI by varying the magnetic field orientation, magnetic field strength, and the incident shock wave Mach number (acceleration strength). The cylindrical interface was selected as its evolution is independent of the orientation of either the shock wave or the magnetic field direction. Instead, the evolution depends only on the orientation of the shock transit direction relative to the magnetic field. The magnetic field parameters were selected to explore their effects on vorticity advection caused by MHD waves, while the Mach number was selected to explore how increasing baroclinic vorticity deposition may effect the growth of the MHD RMI. It was shown that interface mixing is a function of the amount of vorticity deposition, the rate at which vorticity is advected, and the advection direction via Alfven waves. This leads to a rich parameter space where some counterintuitive results are found in mixing measures. To understand the parameter space the 
simulations were observed qualitatively via species and vorticity fields and quantitatively through mixedness, enstrophy, and the correlation coefficient of the vorticity and species gradient. It was found that in general the presence of the magnetic field suppresses RMI-like evolution by the advection of vorticity, with a stronger field, and preferential field orientation resulting in greater suppression. Overall, it was shown that interface mixing is a function of the amount of vorticity deposition, the rate at which it is advected, and the direction it is advected by Alfven waves.

Expanding upon results found in literature, it was shown here that the presence of either a perpendicular or parallel magnetic field in the MHD RMI suppresses RMI evolution; with a perpendicular magnetic field showing less mixedness and significantly reduced RMI morphology compared its parallel field counterpart. This is because in the presence of a magnetic field the whole of the deposited baroclinic vorticity, which drives the evolution in the RMI, no longer remains on the density gradient. This interaction, shown by the species-gradient-vorticity correlation, can be seen to be significantly reduced for the MHD RMI cases when compared to the traditional RMI. The differences in field orientation are further highlighted by the vorticity field and enstrophy. The vorticity is stretched significantly in the direction of the magnetic field, hence the parallel field stretches the vorticity along the shock wave direction. Enstrophy versus time curves, for all enstrophy and enstrophy only near the interface show that both orientations result in lower total enstrophy but that the perpendicular orientation has much lower enstrophy near the interface. Furthermore, vorticity is able to traverse the interface rapidly in the perpendicular field orientation, allowing positive and negative vorticity from opposite sides of the interface to interact and compete, diminishing the enstrophy.

As the magnetic fields are the driving mechanism for the vorticity transport it follows then that as the magnetic field strength increases so does the suppression of 
the RMI evolution. However, by examining magnetic fields of 100, 250, and $500 \mathrm{G}$ in both orientations two nonobvious effects were observed. First, there appears to be some limit to the effectiveness of the magnetic field on mixing. That is, for both field orientations increasing the magnetic field from 250 to $500 \mathrm{G}$ did not significantly affect the mixedness. However, the vorticity and species fields displayed differences in morphology. Secondly, a competing effect was observed for the perpendicular field of 100 G. Here the magnetic field was unable to remove the vorticity from the interface but instead moved it in such a way as to cause significant morphological differences. This interface appears much more diffusive and exhibits higher mixedness and correlation than any other case with $M=1.66$. This implies that while strong fields have been shown to suppress the RMI there may be a regime where magnetic fields can be used to redistribute vorticity through an interface and increase mixing. This effect is likely unique to the perpendicular orientation and the cylindrical interface.

While the magnetic fields drive the MHD effects, the mach number drives the hydrodynamics and exploring the initial Mach number in this work revealed another competing effect. Its known that for the RMI, an increasing Mach number leads to increased interface mixing due to the increased baroclinic vorticity deposition. However, the opposite was observed for the MHD RMI. Qualitatively, the higher Mach number cases further compressed the interface in the MHD RMI. However, this actively drove down mixedness by compressing the diffusion layer, the area where mixing could occur. While the stronger deposited vorticity worked to compress the interface it was advected away by higher Alfven velocities, due to the higher postshock density. Despite this, it was also shown that the stronger vorticity deposition resulted in a more correlated but unmixed interface. Thus the higher Mach number cases exhibited less mixedness and higher vortex advection in MHD.

This work has shown that interface mixing is a function of the amount of vorticity 
deposition, the rate at which it is advected, and the direction it is advected by Alfven waves. This leads to a rich parameter space where some counterintuitive results are found in mixing measures

\subsection{D Simulations of the Cylindrical Magnetohy- drodynamic Richtmyer-Meskhov Instability}

This work studied the 3D cylindrical MHD RM instability by varying the magnetic field orientation relative to the shock transit direction. The cylindrical interface was selected as its evolution is independent of the orientation of either the shock wave or the magnetic field direction. Instead, the evolution depends only on the orientation of the shock transit direction relative to the magnetic field. It was shown that interface evolution is a function of the amount of enstrophy, the growth of the vorticity field, and the advection direction via Alfven waves. This leads to a rich parameter space where some counterintuitive results are found in mixing measures. To understand the parameter space the simulations were observed qualitatively via species contours, as well as temperature and vorticity fields and quantitatively through enstrophy and the correlation coefficient of the vorticity and species gradient. It was found that in general the presence of the magnetic field suppresses RM instability-like evolution by the advection of vorticity with a preferential field orientation resulting in greater suppression.

Expanding upon results found in literature, it was shown here that the presence of either a perpendicular or parallel magnetic field in the MHD RM instability suppresses RM instability evolution; with a perpendicular magnetic field showing less correlation and significantly reduced RM instability morphology compared its parallel field counterpart. This is because in the presence of a magnetic field the whole of 
the deposited baroclinic vorticity, which drives the evolution in the RM instability, no longer remains on the density gradient. The differences in field orientation are further highlighted by the vorticity field and enstrophy. The vorticity is stretched significantly in the direction of the magnetic field, hence the parallel field stretches the vorticity along the shock wave direction. Enstrophy versus time curves, for total enstrophy and the enstrophy components show that the MHD cases result in lower total enstrophy. Furthermore, vorticity is able to traverse the interface rapidly in the perpendicular field orientation, allowing positive and negative vorticity from opposite sides of the interface to interact and compete, diminishing the enstrophy.

Resistivity was also discussed for consideration in future work. It was outlined that the plasma parameters for collisional frequency, electric conductivity, and electron density are immensly important for determining if a simulation requires ideal MHD, resistive MHD, or classic hydrodynamic physics. A model was presented to determine the conductivity assuming a known collisional frequency. It was suggested that for this work a relatively high collisional frequency, on the order of 1 e6 collisions per second, may lead to resistive effects being important.

This document details research performed towards the completion of a Doctoral degree in Mechanical Engineering. For this work, an atmospheric plasma torch was developed for use in future hydrodynamic experiments. The operating conditions of the torch were explored to find optimal power settings for laminar flow and maximum height. The electrical characterisitcs were explored using both an invasive probe technique and a spectroscopic method. These methods revealed both geometric and electrical charactersitics which could be used to determine the temperature and number density of the plasma field. A qualitative imaging technique is also developed for use in future hydrodynamic experiments. Simulations of the Richtmyer-Meshkov Instability with Magnetohydrodynamic effects are also presented in chapters 3 and 4. 
These simulations study the effects of the magnetic field, the strength and orientation of the magnetic field relative to the shock wave direction, and the Mach Number in $2 \mathrm{D}$ and then the magnetic field orientation in 3D. It was found that in general an increasing magnetic field increases suppression of Richtmyer-Meshkov evolution. The orientation of the magnetic field revealed that with a cylindrical interface, the suppression of the magnetic field can drive very different morphologies with a perpendicular magnetic field resulting in almost no Richtmyer-Meshkov evolution. While these simulations are all ideal, resistivity was also considered for future simulations. It was found that if the ioniziation is relatively low, resulting in a magnetic diffusion time higher than the accoustic time scales, then resistivity may play a large role in the evolution of the Richtmyer-Meshkov instability with Magnetohydrodynamic effects. If the magnetic diffusion time is much higher than the instability, then the evolution will not exhibit any Magnetohydrodynamic effects. 


\section{Bibliography}

[1] Wolfgang J Black and Brandon Morgan. Parametric investigation of transition to turbulence in rayleigh-taylor mixing. Unpublished poster, 2018.

[2] Roy C Allen, Wolfgang J Black, and Jacob A McFarland. Development and diagnosis of an atmospheric pressure plasma torch for investigating magnetohydrodynamic instabilities. Journal of Physics D: Applied Physics, 52(17):175201, 2019.

[3] Wolfgang J Black, Roy C Allen, W. Curtis Maxon, Nicholas Denissen, and Jacob A. McFarland. Magnetohydrodynamic effects in a shock-accelerated gas cylinder. Physical Review Fluids, 2019.

[4] Philip G Drazin. Introduction to hydrodynamic stability, volume 32. Cambridge university press, 2002.

[5] William Thomson. Xlvi. hydrokinetic solutions and observations. The London, Edinburgh, and Dublin Philosophical Magazine and Journal of Science, 42(281):362-377, 1871.

[6] Hermann by Helmholtz. On the discontinuous movements of fluids. Academy of Sciences Berlin, 1868. 
[7] Rayleigh Lord. Investigation of the character of the equilibrium of an incompressible heavy fluid of variable density. Scientific Papers, pages 200-207, 1900.

[8] Geoffrey Taylor. The instability of liquid surfaces when accelerated in a direction perpendicular to their planes. i. Proceedings of the Royal Society of London. Series A. Mathematical and Physical Sciences, 201(1065):192-196, 1950.

[9] Robert D Richtmyer. Taylor instability in shock acceleration of compressible fluids. Communications on Pure and Applied Mathematics, 13(2):297-319, 1960.

[10] EE Meshkov. Instability of the interface of two gases accelerated by a shock wave. Fluid Dynamics, 4(5):101-104, 1969.

[11] Subrahmanyan Chandrasekhar. Hydrodynamic and hydromagnetic stability. Courier Corporation, 2013.

[12] William H Cabot and Andrew W Cook. Reynolds number effects on rayleigh-taylor instability with possible implications for type ia supernovae. Nature Physics, pages 562-568, 2006.

[13] R.E Duff, F. H. Harlow, and C.W. Hirt. Effects of diffusion on interface instability between gases. Physics of Fluids, pages 517-426, 1962.

[14] Harry F Robey, JO Kane, BA Remington, RP Drake, OA Hurricane, H Louis, RJ Wallace, J Knauer, P Keiter, D Arnett, et al. An experimental testbed for the study of hydrodynamic issues in supernovae. Physics of Plasmas, 8(5):24462453, 2001.

[15] CE Niederhaus and Jeffrey W Jacobs. Experimental study of the richtmyermeshkov instability of incompressible fluids. Journal of Fluid Mechanics, 485:243-277, 2003. 
[16] PR Chapman and JW Jacobs. Experiments on the three-dimensional incompressible richtmyer-meshkov instability. Physics of Fluids, 18(7):074101, 2006.

[17] Devesh Ranjan, John Niederhaus, Bradley Motl, Mark Anderson, Jason Oakley, and Riccardo Bonazza. Experimental investigation of primary and secondary features in high-mach-number shock-bubble interaction. Physical review letters, 98(2):024502, 2007.

[18] C Tomkins, K Prestridge, P Rightley, M Marr-Lyon, P Vorobieff, and R Benjamin. A quantitative study of the interaction of two richtmyer-meshkovunstable gas cylinders. Physics of Fluids, 15(4):986-1004, 2003.

[19] CD Tomkins, BJ Balakumar, G Orlicz, KP Prestridge, and JR Ristorcelli. Evolution of the density self-correlation in developing richtmyer-meshkov turbulence. Journal of Fluid Mechanics, 735:288-306, 2013.

[20] GC Orlicz, BJ Balakumar, CD Tomkins, and KP Prestridge. A mach number study of the richtmyer-meshkov instability in a varicose, heavy-gas curtain. Physics of Fluids, 21(6):064102, 2009.

[21] Bradley Motl, Jason Oakley, Devesh Ranjan, Chris Weber, Mark Anderson, and Riccardo Bonazza. Experimental validation of a richtmyer-meshkov scaling law over large density ratio and shock strength ranges. Physics of Fluids, 21(12):126102, 2009.

[22] CC Long, VV Krivets, JA Greenough, and JW Jacobs. Shock tube experiments and numerical simulation of the single-mode, three-dimensional richtmyermeshkov instability. Physics of Fluids, 21(11):114104, 2009. 
[23] MA Jones and JW Jacobs. A membraneless experiment for the study of richtmyer-meshkov instability of a shock-accelerated gas interface. Physics of Fluids, 9(10):3078-3085, 1997.

[24] Christopher Weber, Nicholas Haehn, Jason Oakley, David Rothamer, and Riccardo Bonazza. Turbulent mixing measurements in the richtmyer-meshkov instability. Physics of Fluids, 24(7):074105, 2012.

[25] Jacob McFarland, David Reilly, Skylar Creel, Christopher McDonald, Thomas Finn, and Devesh Ranjan. Experimental investigation of the inclined interface richtmyer-meshkov instability before and after reshock. Experiments in fluids, 55(1):1640, 2014.

[26] David Reilly, Jacob McFarland, Mohammad Mohaghar, and Devesh Ranjan. The effects of initial conditions and circulation deposition on the inclinedinterface reshocked richtmyer-meshkov instability. Experiments in Fluids, 56(8):168, 2015.

[27] Jacob A McFarland, David Reilly, Wolfgang Black, Jeffrey A Greenough, and Devesh Ranjan. Modal interactions between a large-wavelength inclined interface and small-wavelength multimode perturbations in a richtmyer-meshkov instability. Physical Review E, 92(1):013023, 2015.

[28] Mohammad Mohaghar, John Carter, Benjamin Musci, David Reilly, Jacob McFarland, and Devesh Ranjan. Evaluation of turbulent mixing transition in a shock-driven variable-density flow. Journal of Fluid Mechanics, 831:779-825, 2017. 
[29] AR Miles, DG Braun, MJ Edwards, HF Robey, RP Drake, and DR Leibrandt. Numerical simulation of supernova-relevant laser-driven hydro experiments on omega. Physics of plasmas, 11(7):3631-3645, 2004.

[30] AR Miles, B Blue, MJ Edwards, JA Greenough, JF Hansen, HF Robey, RP Drake, C Kuranz, and DR Leibrandt. Transition to turbulence and effect of initial conditions on three-dimensional compressible mixing in planar blast-wave-driven systems. Physics of plasmas, 12(5):056317, 2005.

[31] FF Grinstein, AA Gowardhan, and AJ Wachtor. Simulations of richtmyermeshkov instabilities in planar shock-tube experiments. Physics of Fluids, 23(3):034106, 2011.

[32] AM Rasmus, CA Di Stefano, KA Flippo, FW Doss, JL Kline, JD Hager, EC Merritt, TR Desjardins, WC Wan, T Cardenas, et al. Shock-driven discrete vortex evolution on a high-atwood number oblique interface. Physics of Plasmas, 25(3):032119, 2018.

[33] N. Attal and P. Ramaprabhu. Numerical investigation of a single-mode chemically reacting Richtmyer-Meshkov instability. Shock Waves, 25(4):307-328, July 2015.

[34] Jacob A McFarland, Jeffrey A Greenough, and Devesh Ranjan. Simulations and analysis of the reshocked inclined interface richtmyer-meshkov instability for linear and nonlinear interface perturbations. Journal of Fluids Engineering, 136(7):071203, 2014.

[35] Marco Latini, Oleg Schilling, and Wai Sun Don. High-resolution simulations and modeling of reshocked single-mode richtmyer-meshkov instability: Comparison 
to experimental data and to amplitude growth model predictions. Physics of Fluids, 19(2):024104, 2007.

[36] Britton J Olson and Jeffrey A Greenough. Comparison of two-and threedimensional simulations of miscible richtmyer-meshkov instability with multimode initial conditions. Physics of Fluids, 26(10):101702, 2014.

[37] M. T. Henry de Frahan, P. Movahed, and E. Johnsen. Numerical simulations of a shock interacting with successive interfaces using the Discontinuous Galerkin method: the multilayered Richtmyer-Meshkov and Rayleigh-Taylor instabilities. Shock Waves, November 2014.

[38] Takayoshi Sano, Katsunobu Nishihara, Chihiro Matsuoka, and Tsuyoshi Inoue. Magnetic field amplification associated with the richtmyer-meshkov instability. The Astrophysical Journal, 758(2):126, 2012.

[39] Takayoshi Sano, Tsuyoshi Inoue, and Katsunobu Nishihara. Critical magnetic field strength for suppression of the richtmyer-meshkov instability in plasmas. Physical review letters, 111(20):205001, 2013.

[40] M Hohenberger, P-Y Chang, G Fiksel, JP Knauer, R Betti, FJ Marshall, DD Meyerhofer, FH Séguin, and RD Petrasso. Inertial confinement fusion implosions with imposed magnetic field compression using the omega laser. Physics of Plasmas, 19(5):056306, 2012.

[41] Matthew R Gomez, Stephen A Slutz, Adam B Sefkow, Daniel B Sinars, Kelly D Hahn, Stephanie B Hansen, Eric C Harding, Patrick F Knapp, Paul F Schmit, Christopher A Jennings, et al. Experimental demonstration of fusionrelevant conditions in magnetized liner inertial fusion. Physical review letters, 113(15):155003, 2014. 
[42] Adam B Sefkow, SA Slutz, JM Koning, MM Marinak, KJ Peterson, DB Sinars, and RA Vesey. Design of magnetized liner inertial fusion experiments using the z facility. Physics of Plasmas, 21(7):072711, 2014.

[43] PY Chang, G Fiksel, M Hohenberger, JP Knauer, R Betti, FJ Marshall, DD Meyerhofer, FH Séguin, and RD Petrasso. Fusion yield enhancement in magnetized laser-driven implosions. Physical review letters, 107(3):035006, 2011.

[44] SA Slutz, MC Herrmann, RA Vesey, AB Sefkow, DB Sinars, DC Rovang, KJ Peterson, and ME Cuneo. Pulsed-power-driven cylindrical liner implosions of laser preheated fuel magnetized with an axial field. Physics of Plasmas, 17(5):056303, 2010.

[45] Stephen A Slutz and Roger A Vesey. High-gain magnetized inertial fusion. Physical review letters, 108(2):025003, 2012.

[46] A. Schutze, J. Y. Jeong, S. E. Babayan, , G. S. Selwyn, and R. F. Hicks. The atmospheric-pressure plasma jet: a review and comparison to other plasma sources. IEEE Transactions on Plasma Science, 26(6):1685-1694, Dec 1998.

[47] Ravi Samtaney. Suppression of the richtmyer-meshkov instability in the presence of a magnetic field. Physics of Fluids, 15(8):L53-L56, 2003.

[48] Vincent Wheatley, DI Pullin, and R Samtaney. Stability of an impulsively accelerated density interface in magnetohydrodynamics. Physical review letters, 95(12):125002, 2005.

[49] Vincent Wheatley, R Samtaney, and DI Pullin. The richtmyer-meshkov instability in magnetohydrodynamics. Physics of Fluids, 21(8):082102, 2009. 
[50] V Wheatley, Ravi Samtaney, DI Pullin, and RM Gehre. The transverse field richtmyer-meshkov instability in magnetohydrodynamics. Physics of Fluids, 26(1):016102, 2014.

[51] F. Francis Chen et al. Introduction to plasma physics and controlled fusion. Plasma Physics, 1984.

[52] W. Mostert, V. Wheatley, R. Samtaney, and D. I. Pullin. Effects of seed magnetic fields on magnetohydrodynamic implosion structure and dynamics. Physics of Fluids, 26(12):126102, December 2014.

[53] W Mostert, V Wheatley, Ravi Samtaney, and DI Pullin. Effects of magnetic fields on magnetohydrodynamic cylindrical and spherical richtmyer-meshkov instability. Physics of Fluids, 27(10):104102, 2015.

[54] Wenxia Pan, Wenhong Zhang, Wei Ma, and Chengkang Wu. Characteristics of argon laminar dc plasma jet at atmospheric pressure. Plasma Chemistry and Plasma Processing, 22(2):271-283, 2002.

[55] A M Paingankar, A K Das, V S Shirodkar, K P Sreekumar, and N Venkatramani. Prediction of electrical characteristics of a non-transferred arc-plasma torch using principles of dynamic similarity. Plasma Sources Science and Technology, 8(1):100-109, jan 1999.

[56] Gang Li, Wenxia Pan, Xian Meng, and Chengkang Wu. Application of similarity theory to the characterization of non-transferred laminar plasma jet generation. Plasma Sources Science and Technology, 14(2):219-225, feb 2005. 
[57] M. P. Planche, J. F. Coudert, and P. Fauchais. Velocity measurements for arc jets produced by a dc plasma spray torch. Plasma Chemistry and Plasma Processing, 18(2):263-283, Jun 1998.

[58] A. Vardelle, P. Fauchais, B. Dussoubs, and N. J. Themelis. Heat generation and particle injection in a thermal plasma torch. Plasma Chemistry and Plasma Processing, 18(4):551-574, Dec 1998.

[59] Irving Langmuir. Scattering of electrons in ionized gases. Phys. Rev., 26:585613, Nov 1925.

[60] L. Prevosto, H Kelly, and B. R. Mancinelli. Langmuir probe diagnostics of an atmospheric pressure, vortex-stabilized nitrogen plasma jet. Journal of Applied Physics, 112, 2012.

[61] Jeremiah Williams. A simple, inexpensive langmuir probe experiment. Unpublished.

[62] P. R Smy. The use of langmuir probes in the study of high pressure plasmas. Advances in Physics, 25(5):517-553, 1976.

[63] C Fanara and I M Richardson. A langmuir multi-probe system for the characterization of atmospheric pressure arc plasmas. Journal of Physics D: Applied Physics, 34(18):2715-2725, sep 2001.

[64] Robert S Powers. Comparison of langmuir probe and spectrometric electron temperature measurements. Journal of Applied Physics, 37:3821-3826, 1966.

[65] Yong-ik Sung, H. B Lim, and R. S. Houk. Diagnostic studies of a low-pressure inductively coupled plasma in argon using a double langmuir probe. Journal of Analytical Atomic Spectrometry, 6, 2002. 
[66] Lu Pei-Qi, Gong Pei-Zhong, Lin Tie-Zheng, and R. S. Houk. Langmuir probe measurements of electron temperature in an inductively coupled plasma. Journal of Applied Physics, 43:273-285, 1988.

[67] Sin-Li Chen and T. Sekiguchi. Instantaneous direct-display system of plasma parameters by means of triple probe. Journal of Applied Physics, 36:2363-2375, 1965.

[68] A F H van Gessel, E A D Carbone, P J Bruggeman, and J J A M van der Mullen. Laser scattering on an atmospheric pressure plasma jet: disentangling rayleigh, raman and thomson scattering. Plasma Sources Science and Technology, 21(1):015003, jan 2012.

[69] Gregoire de Izarra, Nuno Cerqueira, and Charles de Izarra. Quantitative shadowgraphy on a laminar argon plasma jet at atmospheric pressure. Journal of Physics D: Applied Physics, 44(48):485202, nov 2011.

[70] Yashuang Zheng, Lijun Wang, Wejun Ning, and Shenli Jia. Schlieren imaging investigation of the hydrodynamics of atmospheric helium plasma jets. Journal of Applied Physics, 119, 2016.

[71] B Pokrzywka, K Musiol, S Pellerin, E Pawelec, and J Chapelle. Spectroscopic investigation of the equilibrium state in the electric arc cathode region. Journal of Physics D: Applied Physics, 29(10):2644-2649, oct 1996.

[72] N K Joshi, S N Sahasrabudhe, K P Sreekumar, and N Venkatramani. Variation of axial temperature in thermal plasma jets. Measurement Science and Technology, 8(10):1146-1150, oct 1997. 
[73] Simon Hübner, Joao Santos Sousa, Joost van der Mullen, and William G Graham. Thomson scattering on non-thermal atmospheric pressure plasma jets. Plasma Sources Science and Technology, 24(5):054005, jan 2017.

[74] S.J Zweben, D.P Stotler, J. L Terry, B. LaBombard, M. Greenwald, M Muterspaugh, C. S Pitcher, Alcator C-Mod Group, K Hallatschek, R. J. Maqueda, and B Rogets. Edge turbulence imaging in the alcator c-mod tokamak. Journal of Physics D: Applied Physics, 9(5):1971-1989, 2002.

[75] A. B Murphy. Modified fowler-milne method for the spectroscopic measurement of temperature and composition of multielement thermal plasmas. Review of Scientific Instruments, 65(11):3423-3427, 1994.

[76] J Haidar and A J D Farmer. Temperature measurements for high-current freeburning arcs in nitrogen. Journal of Physics D: Applied Physics, 26(8):12241229, aug 1993.

[77] D. A. Scott, P. Kovitya, and G. N Haddad. Temperatures in the plume of a dc plasma torch. Journal of Applied Physics, 66:5232-5239, 1989.

[78] B Bachmann, R Kozakov, G Gött, K Ekkert, J-P Bachmann, J-L Marques, H Schöpp, D Uhrlandt, and J Schein. High-speed three-dimensional plasma temperature determination of axially symmetric free-burning arcs. Journal of Physics D: Applied Physics, 46(12):125203, 2013.

[79] R Kozakov, B Emde, A V Pipa, M Huse, D Uhrlandt, J Hermsdorf, and V Wesling. Change of electrical conductivity of ar welding arc under resonant absorption of laser radiation. Journal of Physics D: Applied Physics, 48(9):095502, feb 2015. 
[80] R. H Fowler and E. A Milne. The intensities of absorption lines in stellar spectra, and the temperatures and pressures in the reversing layers of stars. Monthly Notices of the Royal Astronomical Society, 83:403-424, 1923.

[81] G N Haddad and A J D Farmer. Temperature determinations in a free-burning arc. i. experimental techniques and results in argon. Journal of Physics D: Applied Physics, 17(6):1189-1196, jun 1984.

[82] A J D Farmer and G N Haddad. Rayleigh scattering measurements in a freeburning argon arc. Journal of Physics D: Applied Physics, 21(3):426-431, mar 1988.

[83] Nityalendra Singh, Manitra Razafinimanana, and Alain Gleizes. The effect of pressure on a plasma plume: temperature and electron density measurements. Journal of Physics D: Applied Physics, 31(20):2921-2928, oct 1998.

[84] John B. Middlebrooks, Constantine Avgoustopoulos, Wolfgang J. Black, Roy C. Allen, and Jacob. McFarland. Droplet and multiphase effects in a shock-driven hydrodynamic instability with reshock experiments in fluids. Experiments in Fluids, XX(X):X, 2018.

[85] Wenxia Pan, Wenhua Zhang, Wenhong Zhang, and Chengkang Wu. Generation of long, laminar plasma jets at atmospheric pressure and effects of flow turbulence. Plasma Chemistry and Plasma Processing, 21(1):23-35, Mar 2001.

[86] A K Das, K P Sreekumar, and N Venkatramani. DC plasma torch voltage and current characteristics through heat balance measurements. Plasma Sources Science and Technology, 3(1):108-113, feb 1994. 
[87] Wenxia Pan, Xian Meng, Chengkang Wu, and Xi Chen. Experimental study on the thermal argon plasma generation and jet length change characteristics at atmospheric pressure. Plasma Chemistry and Plasma Processing, 26(4):335345, Aug 2006.

[88] V Valin ius, V Kru inskaite, P Valatkevi ius, V Valin i te, and L Marcinauskas. Electric and thermal characteristics of the linear, sectional dc plasma generator. Plasma Sources Science and Technology, 13(2):199-206, feb 2004.

[89] Gianpiero Colonna and Mario Capitelli. A few level approach for the electronic partition function of atomic systems. Spectrochimica Acta Part B: Atomic Spectroscopy, 64(9):863 - 873, 2009.

[90] Y T Cho and S-J Na. Application of abel inversion in real-time calculations for circularly and elliptically symmetric radiation sources. Measurement Science and Technology, 16(3):878-884, feb 2005.

[91] A. Kramida, Y. Ralchenko, J. Reader, and NIST ASD Team. Nist: Atomic spectra database (version 5.5.6). Nist Atomic Spectra Database (Online).

[92] WJ Black, N Denissen, and JA McFarland. Particle force model effects in a shock-driven multiphase instability. Shock Waves, pages 1-10, 2017.

[93] Jeevan Dahal and Jacob A. McFarland. A numerical method for shock driven multiphase flow with evaporating particles. Journal of Computational Physics, $344: 210-233,2017$.

[94] Donald E Burton. Connectivity structures and differencing techniques for staggered-grid free-lagrange hydrodynamics. Lawrence Livermore National Laboratory, Report No. UCRL-JC-110555, 1992. 
[95] Donald E Burton. Consistent finite-volume discretization of hydrodynamic conservation laws for unstructured grids. Lawrence Livermore National Laboratory, Report No. UCRL-JC-118788, 1994.

[96] Jay P Boris and David L Book. Flux-corrected transport. i. shasta, a fluid transport algorithm that works. Journal of computational physics, 11(1):38-69, 1973. doi:10.1016/0021-9991(73)90147-2.

[97] Jimmy Fung, Alan K Harrison, Shirish Chitanvis, and Jeremy Margulies. Ejecta source and transport modeling in the flag hydrocode. Computers \& Fluids, 83:177-186, 2013. doi:10.1016/j.compfluid.2012.08.011.

[98] AK Harrison and J Fung. Ejecta in the flag hydrocode. Numerical Methods for Multi-Material Fluids and Structures, Pavia, 2009.

[99] Steven T Zalesak. Fully multidimensional flux-corrected transport algorithms for fluids. Journal of computational physics, 31(3):335-362, 1979. doi:10.1016/0021-9991(79)90051-2.

[100] Timothy Barth and Dennis Jespersen. The design and application of upwind schemes on unstructured meshes. In 27th Aerospace sciences meeting, page 366, 1989.

[101] David L Youngs. Time-dependent multi-material flow with large fluid distortion. Numerical methods for fluid dynamics, 24(2):273-285, 1982.

[102] V Wheatley, DI Pullin, and R Samtaney. Regular shock refraction at an oblique planar density interface in magnetohydrodynamics. Journal of Fluid Mechanics, 522:179-214, 2005. 
[103] BE Morgan, BJ Olson, JE White, and JA McFarland. Self-similarity of a rayleigh-taylor mixing layer at low atwood number with a multimode initial perturbation. Journal of Turbulence, 18(10):973-999, 2017.

[104] Britton J Olson and Jeff Greenough. Large eddy simulation requirements for the richtmyer-meshkov instability. Physics of Fluids, 26(4):044103, 2014.

[105] BE Morgan and JA Greenough. Large-eddy and unsteady rans simulations of a shock-accelerated heavy gas cylinder. Shock Waves, 26(4):355-383, 2016.

[106] Santhosh K Shankar, Soshi Kawai, and Sanjiva K Lele. Two-dimensional viscous flow simulation of a shock accelerated heavy gas cylinder. Physics of Fluids, 23(2):024102, 2011.

[107] V Gregory Weirs, Todd Dupont, and Tomasz Plewa. Three-dimensional effects in shock-cylinder interactions. Physics of Fluids, 20(4):044102, 2008.

[108] RW Sharp Jr and RT Barton. Hemp advection model. Technical report, California Univ., Livermore (USA). Lawrence Livermore Lab., 1981.

[109] J Donea, Antonio Huerta, J-Ph Ponthot, and A Rodriguez-Ferran. Arbitrary lagrangian-eulerian methods, volume 1 of encyclopedia of computational mechanics, chapter 14. John Wiley \&3 Sons Ltd, 3:1-25, 2004.

[110] Mark L Wilkins. Calculation of elastic-plastic flow. Technical report, California Univ Livermore Radiation Lab, 1963.

[111] Hank Childs, Eric Brugger, Brad Whitlock, Jeremy Meredith, Sean Ahern, David Pugmire, Kathleen Biagas, Mark Miller, Cyrus Harrison, Gunther H. Weber, Hari Krishnan, Thomas Fogal, Allen Sanderson, Christoph Garth, E. Wes Bethel, David Camp, Oliver Rübel, Marc Durant, Jean M. Favre, and Paul 
Navrátil. VisIt: An End-User Tool For Visualizing and Analyzing Very Large Data. In High Performance Visualization-Enabling Extreme-Scale Scientific Insight, pages 357-372. Oct 2012.

[112] Lee Ellison. Magnetic diffusion and resistivity. Private Communication and Notes on Magnetohydrodynamics. 


\section{VITA}

Wolfgang Justice Black was born in Denver Colorado. He completed a Bachelors of Science in Mechanical Engineering with minors in Mathematics and East Asian Studies at the University of Missouri, graduating in May 2014. In August 2016 Wolfgang received a Masters of Science in Mechical Engineering from MU for his thesis work, "A Preliminary Study of Shock Driven Multiphase Hydrodynamic Instabilities". Finally he received a Doctoral Degree also in Mechanical Engineering from MU for his work, "Study of Magnetohydrodynamic Effects for the Richtmyer-Meshkov Instability". Wolfgang began working with Dr. Jacob McFarland in the Fall Semester of 2014 where he helped to form the Missouri Shock Tube and Fluid Mixing laboratory as it's only student member. His work has included the design of experimental facilities, creation of experimental diagnostics, developement of experimental processes, simulating hydrodynamic systems through the use of multi-physics hydrocodes. He has worked at Los Alamos and Lawrence Livermore National Labs during his tenure as a graduate student at the University of Missouri. Wolfgang start his career outside of academia in September 2018 working for JUUL Labs in San Francisco, California as it's first Fluid Dynamics Researcher. As of March 2019 he designs experiments, creates processes, and uses proprietary software to analyse the physics involved in current and future products at JUUL Labs. 